\title{
Multiphase-field model for surface diffusion and attachment kinetics in the grand-potential framework
}

\author{
Paul W. Hoffrogge, ${ }^{1}$ Arnab Mukherjee, ${ }^{2}$ E. S. Nani, ${ }^{1}$ P. G. Kubendran Amos,,${ }^{1,3}$ Fei Wang, ${ }^{1}$ \\ Daniel Schneider, ${ }^{1,4}$ and Britta Nestler ${ }^{1,4}$ \\ ${ }^{1}$ Institute of Applied Materials-Computational Materials Science, Karlsruhe Institute of Technology, Strasse am Forum 7 , \\ 76131 Karlsruhe, Germany \\ ${ }^{2}$ Center for Hierarchical Materials Design, Northwestern University, 2205 Tech Drive, Evanston, Illinois 60208, USA \\ ${ }^{3}$ Department of Metallurgical and Materials Engineering, National Institute of Technology, Tiruchirappalli 620015, Tamil Nadu, India \\ ${ }^{4}$ Institute of Digital Materials Science, Karlsruhe University of Applied Sciences, Moltkestr. 30, 76133 Karlsruhe, Germany
}

(Received 19 February 2020; revised 16 November 2020; accepted 12 March 2021; published 29 March 2021)

\begin{abstract}
Grand-potential based multiphase-field model is extended to include surface diffusion. Diffusion is elevated in the interface through a scalar degenerate term. In contrast to the classical Cahn-Hilliard-based formulations, the present model circumvents the related difficulties in restricting diffusion solely to the interface by combining two second-order equations, an Allen-Cahn-type equation for the phase field supplemented with an obstacletype potential and a conservative diffusion equation for the chemical potential or composition evolution. The sharp interface limiting behavior of the model is deduced by means of asymptotic analysis. A combination of surface diffusion and finite attachment kinetics is retrieved as the governing law. Infinite attachment kinetics can be achieved through a minor modification of the model, and with a slight change in the interpretation, the same model handles the cases of pure substances and alloys. Relations between model parameters and physical properties are obtained which allow one to quantitatively interpret simulation results. An extensive study of thermal grooving is conducted to validate the model based on existing theories. The results show good agreement with the theoretical sharp-interface solutions. The obviation of fourth-order derivatives and the usage of the obstacle potential make the model computationally cost-effective.
\end{abstract}

DOI: 10.1103/PhysRevE.103.033307

\section{INTRODUCTION}

The microstructure of a material reflects the morphological configuration of the constituent phases and grains. Effective material properties are directly related to the underlying microstructure. Therefore, naturally, to understand and control the behavior of the material under various conditions, the microstructural evolution is extensively analyzed. During the evolution, numerous physical processes may be coupled inherently. In addition to the diffusion of the involved chemical species, the microstructural transformation is prominently driven by interfacial energy and mechanical stresses and strains. To untangle the inherent complexities in the temporal evolution of a microstructure, theoretical treatments are employed. Phase-field modeling is one such numerical technique, which is widely gaining ground for its ability to describe a thermodynamically consistent microstructural evolution.

\section{A. Phase-field framework}

The distribution of phases and grains in a microstructure typically exhibits complex geometries and topologies. More precisely, when the microstructure evolves with time, the migration of interfaces may lead to a coalescence and/or a breakup of particles, and interfaces and junctions may form or disappear. To explicitly track all possible events, while the microstructure is altered, turns into an arduous task. Phasefield approaches [1-4] circumvent these difficulties by introducing a scalar and dimensionless variable, the phase field $\phi(\boldsymbol{x}, t)$, which exhibits spatiotemporal dependency. Through a spatial variation of the phase field, the domain is decomposed into different bulk regions and interfaces. Instead of explicitly implementing an equation of motion for the interface, the temporal behavior is delineated implicitly through a partial differential equation for the phase-field variable. The tracking of the interface is obviated, because all possible morphological transformations are properly described by solving the imposed equation. However, due to the implicit treatment, additional effort lies in demonstrating the ability to recover the conventional (and explicit) sharp interface solutions and physical laws.

The phase field, defined in this form, can be interpreted as the local volume fraction of the corresponding phase. The interface thus corresponds to the region of a rapidly varying phase field. Therefore, the sharp interface in phase-field models is replaced by a diffuse region of well-defined thickness. The phase field $\phi(\boldsymbol{x}, t)$ is usually nonconserved, i.e., one phase may freely transform into the other, as a result of a driving force acting on the interface between them. Such a process is commonly incorporated, following Allen and Cahn [5], where the evolution of the phase field leads to a reduction in the free energy of the system. These sorts of evolution equations are 
therefore termed Allen-Cahn equations. Following Cahn and Hilliard [6], the phase field is alternatively interpreted as a composition (i.e., mole fraction of one of the species), written as $c(\boldsymbol{x}, t)$, which is a conserved quantity, and consequently augmented with a diffusion equation. Models following this approach are therefore termed Cahn-Hilliard models.

Both approaches start with a formulation of the overall free energy $F$ of the system, in terms of either $\phi$ or $c$. The capillarity effect is naturally included in the models through a gradient and a potential term, which balance each other to form a well-defined interfacial profile. In an Allen-Cahn model, the motion of the interface is proportional to the variation of the free energy, i.e., $\dot{\phi} \propto \delta F / \delta \phi$, while in a Cahn-Hilliard model, spatial differences lead to a flux in the free energy variation, whose divergence governs the evolution, such that $\dot{c} \propto \nabla^{2} \delta F / \delta c$, where $\nabla^{2}$ is the Laplacian. Numerous problems require a coupling of both notions in a single model, such as solidification, where diffusion and phase transformation happen simultaneously, or sintering, where concomitant grain growth and coarsening takes place. Questions arise as to whether $c$ or $\phi$ should be used to include interfacial energy into the functional or both of them. Additional questions appear, concerning the quantitative treatment of the bulk properties and the extension of the model for a multicomponent setup. Since starting from initial ideas [7-9], these questions have been the scope of numerous works, [10-13], in which tremendous progress has been made. One of the promising candidates is the grand potential model $[14,15]$, extended in this work. Apart from the coupling of diffusion and phase transformations, a scalar phase field can be used only to distinguish two distinct phases. The phase-field technique had to be extended to delineate systems comprising more than two phases (e.g., polycrystalline materials). In such multiphasefield models [16-18], the scalar variable is replaced by a vector quantity, $\phi=\left\{\phi_{\alpha}, \phi_{\beta}, \ldots, \phi_{N}\right\}$, to efficiently describe the contribution of each of the $N$ multiple phases. The bulk is characterized by a constant value of the phase field $\left(\left|\nabla \phi_{\alpha}\right|=\right.$ $0, \forall \alpha \in\{1, \ldots, N\}, \nabla$ denotes the gradient). In the interface, which separates the bulk phases, the phase field exhibits a smooth monotonic transition $\left(\left|\nabla \phi_{\alpha}\right| \neq 0, \alpha \in\{1, \ldots, N\}\right)$. Moreover, apart from bulk diffusion, some material systems are strongly governed by rapid diffusion along the interfaces, such as, for example, during thermal grooving $[19,20]$. The incorporation of surface diffusion in phase-field models requires additional terms to be included in the model. Since the current work focuses on this particular subject, the historical progress for surface diffusion, in the field of phase-field models, is explained in detail in the following section.

\section{B. Surface diffusion in phase-field models \\ 1. Fourth-order conservative equation [Cahn-Hilliard (CH) equation]}

In the context of phase-field models, it was first proposed by Cahn and Taylor [21] that the Cahn-Hilliard equation with a suitable composition-dependent mobility leads to a diffuse interface counterpart of motion by surface diffusion, or to motion by means of the surface Laplacian of the mean curvature.
The governing equations write as

$$
\begin{aligned}
& \frac{\partial c}{\partial t}=\nabla \cdot[M(c) \nabla \omega], \\
& \omega=\frac{\partial f(c)}{\partial c}-\epsilon^{2} \nabla^{2} c,
\end{aligned}
$$

where $c$ is a composition, $\omega$ is a chemical potential, and $\epsilon$ is a parameter related to the interface width. $\nabla \cdot$ denotes the divergence operator. Note that all dimensions of time and space, as well as energy, are dimensionless in this form. The mobility function $M(c)$ is chosen in such a way that the diffusion is restricted to the narrow interfacial region. This means that if the potential $f(c)$ is chosen such that 0 and 1 represent the bulk phases $M(c) \neq 0$, for $0<c<1$ and $M(c)=0$ elsewhere.

Two forms of $f(c)$, namely, (i) of the obstacle type [i.e., $f(c)=c(1-c)$ for $0<c<1$ and $\infty$ elsewhere] and (ii) of the well type [i.e., $f(c)=c^{2}(1-c)^{2}$ ], were discussed in conjunction with a quadratic degenerate form of mobility [i.e., $M(c)=c(1-c)$ ]. It was further argued that the choice of a degenerate mobility function restricts atomic transport to the interfacial region, since the obstacle-type free energy drives $c$ to exactly 0 and 1 . With a formal asymptotic analysis, it was later shown by Cahn et al. [22] that such obstacle-type free energy, along with a degenerate mobility, results in interface motion by the surface Laplacian of the mean curvature.

Since then, most of the works have employed a quadratic mobility, in conjunction with a well-type free energy [23-29]. A biquadratic mobility function [i.e., $M(c)=c^{2}(1-c)^{2}$ ] was also introduced in some works [30-32], without discussing a clear rationale. The domain of applicability was extended to study the coarsening of two-phase mixtures [23,24,29,3335], solid-state dewetting [28,36], thin film growth [37-39] and coupled problems of electromigration [40-42], as well as stress-induced surface instability [25,31]. In fact, an asymptotic analysis for a well-type free energy was first performed for a coupled electromigration [40] and mechanics problem [31]. While the latter article employed a biquadratic form of mobility, the former introduced a mobility function of the type $M(c)=\nabla c \cdot \nabla c$. The motion caused by the surface Laplacian of the curvature and the motion caused by the surface Laplacian of the electric potential or stress was recovered in the limit $\epsilon \rightarrow 0$. The numerical results by Lacasta $e t$ al. [33,34], Bray and Elliot [35], Puri et al. [23], Zhu et al. [24], and Dai and Du [29] agree that such a combination does not lead to kinetics limited by pure surface diffusion, but additionally consists of contributions from bulk diffusion. The aforementioned result has recently been proved rigorously and independently by Dai and Du [43] and Lee et al. [44,45], using a matched asymptotic analysis. The analysis clearly reveals that such a combination results in the presence of a bulk diffusion term at the same order as the surface diffusion term. This can be attributed to the fact that in $\mathrm{CH}$ models, $c$ serves a dual purpose of providing the composition in the bulk and tracking the interface. As a result of the Gibbs-Thomson effect, the value of $c$ deviates from the equilibrium values, as $c=c_{\text {eq }}+k \kappa \epsilon / f^{\prime \prime}\left(c_{\text {eq }}\right)$, depending on the curvature, where $k$ is a positive constant and $\kappa$ is the curvature. It was further shown in $[43,45]$ that the contribution of the bulk diffusion flux to the interface velocity is dependent on the derivative of the mobility $M^{\prime}\left(c_{o}\right)$, where $c_{o}$ are the equilibrium values 
in the respective phases. The higher order degeneracy in the mobility function satisfies the criterion $M^{\prime}\left(c_{o}\right)=0$ to negate the unwanted contribution of the bulk diffusion. In the context of solid-state dewetting, numerical comparisons between the quadratic and biquadratic mobilities confirm the mathematical analysis [46]. For a well-type free energy, Rätz, Ribalta and Voigt [32] developed an alternative doubly degenerate model for surface diffusion. The special feature of the model is the presence of a stabilizing function $g(c)$ which, similar to the free energy and mobility, is biquadratic, i.e.,

$$
\begin{gathered}
\frac{\partial c}{\partial t}=\nabla \cdot\left[\frac{M(c)}{\epsilon} \nabla \omega\right], \\
g(c) \omega=\frac{1}{\epsilon} \frac{\partial f(c)}{\partial c}-\epsilon \nabla^{2} c .
\end{gathered}
$$

The term doubly degenerate refers to the degeneracy in both the mobility and the stabilizing function. Although the role of the stabilizing function is not apparent from the asymptotics, it has been shown to perform better in numerical calculations $[32,39,47,48]$. Interestingly, a rescaling of Eqs. (3) and (4), as compared to Eqs. (1) and (2), is evident, since the mobility and the chemical potential were scaled by $\epsilon$. Consequently, the leading-order inner chemical potential in the asymptotic expansion is equal to the curvature. As opposed, Eqs. (1) and (2) recover the Gibbs-Thomson condition as a first-order correction. Our understanding of the recovery of surface diffusion from the fourth-order $\mathrm{CH}$ equation is still evolving. The current understanding of the subject is that a higher degeneracy in mobility is warranted for a biquadratic free energy, so as to suppress the contribution from bulk diffusion, while the combination of obstacle-type free energy and degenerate mobility suffices to recover pure surface diffusion. The role of stabilizing functions is still a topic of an active debate $[49,50]$. In addition to the scalar mobilities, tensorial mobilities have also been proposed by Gugenberger et al. [47], which inherently restrict diffusion along the interface. Using both asymptotic analysis and a numerical example, comparisons of scalar and tensorial mobilities were provided, which revealed a better performance of the latter for certain cases.

\section{Coupled fourth-order conservative [Cahn-Hillard (CH)] and second-order nonconservative [Allen-Cahn (AC)] equation}

There are many problems in materials science, where surface diffusion is often accompanied by surface attachment kinetics [21,51]. In addition, most materials are polycrystalline. The temperature range of interest, where surface diffusion is dominant, leads to concurrent grain growth in multigrain materials. In such settings, the interphase boundary (the interface between two chemically different phases) evolves via surface diffusion, while the grain boundaries migrate by means of curvature. Sintering [52,53], grooving, and pitting in polycrystalline thin films $[54,55]$, solid-state dewetting in polycrystalline materials [56], and grain growth in porous solids $[57,58]$ are a few examples of such a coupled motion. To tackle such problems within the context of phasefield models, the Cahn-Hilliard $(\mathrm{CH})$ equation is coupled to an Allen-Cahn (AC) equation. The conservative variable distinguishes between the interphase boundaries, while the nonconservative variables differentiate between the various grain orientations in the solid. The coupled system of equations writes as

$$
\begin{gathered}
\frac{\partial c}{\partial t}=\nabla \cdot[M(c, \phi) \nabla \omega], \\
\omega=\frac{\partial f(c, \phi)}{\partial c}-\epsilon^{2} \nabla^{2} c, \\
\tau(\epsilon) \frac{\partial \phi}{\partial t}=q \epsilon^{2} \nabla^{2} \phi-\frac{\partial f(c, \phi)}{\partial \phi},
\end{gathered}
$$

where $q$ is a positive constant, introduced to tailor disparate surface and grain boundary energies. $\tau$ is the grain boundary relaxation parameter, which is a function of $\epsilon . f(c, \phi)$ is a typical Landau polynomial, which is a sum of a double well (or obstacle) in $c$, a multiwell (or multiobstacle) in $\phi$, so as to account for different grains in the solid, and a coupling term in $c$ and $\phi[52,54]$. The function $f(c, \phi)$ additionally must satisfy $\partial f(c, \phi) /\left.\partial c\right|_{c=c_{\mathrm{s}}^{\mathrm{eq}}}=0$ at each point inside the solid, including the grain boundaries, to fulfill the condition of a non-adsorption of ghost phases. $c_{s}^{\mathrm{eq}}$ represents the equilibrium value in the solid. The mobility function now is enslaved to both $c$ and $\phi$, to account for disparate mobilities along the surface and grain boundaries. Equations (5) and (7) are known as a system of degenerate Cahn-Hilliard and nondegenerate Allen-Cahn equations, because $\tau$ is independent from the field variables. Since $f(c, \phi)$ is constructed in such a way that $c$ is constant in the solid, in the course of the evolution, the kinetics of the grain boundary is governed by the Allen-Cahn equation (7). Due to the inherent coupling, any motion of the interphase boundary depends on the scaling of the grain boundary relaxation parameter [59]. For $\tau=O(1)$, the surface motion is due to the geometric law, which combines surface diffusion and surface attachment kinetics. For this scaling process, the grain boundary moves through the motion of the mean curvature. To recover a pure motion of the interphase boundary, limited by surface diffusion, $\tau$ has to scale as $\tau=O(\epsilon)$. Accordingly, the grain boundary is in its equilibrium configuration, i.e., the curvature of the grain boundary is zero. In the case of a welltype potential, the restriction of the order of mobility function, to recover a pure motion, limited by surface diffusion, still holds for these systems of equations.

A different version of Eq. (7), along with Eq. (5), namely, a degenerate $\mathrm{CH}$ equation and a degenerate AC equation $(\tau$ is a function of $c$ and $\phi$ ), has been analyzed by NovickCohen and co-workers $[60,61]$. In such systems of equations, one can also derive a case where the interphase boundary moves via surface diffusion and the grain boundary is governed by curvature. These systems of equations have been employed in a multitude of problems, ranging from sintering $[52,62-65]$ to the interaction between pores and grain boundaries [66-68], grain boundary grooving [54,63,69,70], grain growth in porous solids [57,58,71], additive manufacturing processes, such as selective laser sintering [72], and direct ink writing [53].

\section{Coupled second-order conservative and nonconservative equations}

The form of the governing equations, which perhaps has received the least attention in this regard, is a system of a coupled second-order diffusion equation and a second-order 
nonconservative Allen-Cahn equation. The governing set of equations is the same as Eqs. (5) and (7), but without the Laplacian term in the diffusion equation. Since the gradient energy of the conserved variable has been dropped from the free energy functional, an additional phase-field variable has to be introduced to demarcate the interphases. Thus, the phase-field variables are introduced only to differentiate between interphase boundaries and grain boundaries. Hereby, the conservative variable exclusively accounts for the atomic transport.

In such a framework, Amirouche and Plapp [73] presented a model for discontinuous precipitation, based on a well-type potential. While the study did not focus only on surface diffusion, it constitutes one of the many mass transport mechanisms. Among other findings, the authors discussed difficulties regarding the recovery of the Gibbs-Thomson condition, in the case of a strong surface diffusion, since the chemical potential in the interface region remained nonuniform and dependent on the initial condition. Chakraborty et al. [74] studied the phenomenon of surface diffusion and electromigration-induced grain boundary grooving in the grand-potential framework and used an obstacle-type potential. Within a similar approach, Hötzer et al. [75] studied the sintering of particles with surface diffusion as one of the primary mass transport mechanisms, as well as concurrent grain growth. To restrict diffusion along the interfaces, all aforementioned works have considered a scalar mobility function. Greenquist et al. [76,77] have also developed a grand potential-based sintering model, in conjunction with a tensorial mobility approach.

Although numerical results were presented in the abovementioned articles, the convergence of the coupled system of equations to the sharp interface limit has not been shown formally. We aim to bridge the gap by recovering the geometric laws for such a combination of governing equations. If the interfacial motions are recovered, such a system of equations can be a viable alternative to the previously discussed models.

First, a second-order diffusion equation expedites numerical calculation by obviating the need to discretize the additional Laplacian operator at each time step. With an explicit spatial and widely employed discretization scheme, the temporal discretization width $\Delta t$ scales as $(\Delta x)^{4}$, for a fourthorder diffusion equation with the spatial dicretization width, and as $(\Delta x)^{2}$, for a second-order diffusion equation. Thus, for the same spatial discretization width, a higher temporal discretization width can be utilized with these systems of equations, without initiating numerical instabilities.

Second, the presence of the gradient energy term in the conserved order parameter makes the interfacial energy of the free energy functional dependent on the chosen bulk-free energy expression. This dependency not only complicates the parametrization, but inherently couples the interfacial energy and width, which cannot be chosen independently. For the present system of second-order conservative and nonconservative equations, which are based on the grand potential model or on the related Kim-Kim-Suzuki [10] framework, the interfacial energy and width can be set independently. Moreover, the grand potential model supports multiple phases and components in a generic way, because the free energy functions $f_{\alpha}\left(\boldsymbol{c}^{\alpha}\right)$ can be chosen freely, in contrast to $f(c)$ in the Cahn-Hilliard equation.

Finally, the interface in these models is defined by a level set of the phase field, which does not exhibit a GibbsThomson correction. In other words, this implies that the bulk values of the phase field do not deviate from 0 and 1 . Since the mobility function depends on $\boldsymbol{\phi}$, instead of $\boldsymbol{c}$, the restriction, which exists on its order, as in the previous two cases, should not hold here. Therefore, the equilibrium composition has not necessarily to be zero or unity, but can assume arbitrary values in the bulk. Moreover, an extension, formulated in the grand-potential framework, is promising since it inherits the readily available applicability to multiphase and multicomponent systems. Due to these reasons, the hope is that such a surface-diffusion model can be made applicable to a much wider range of materials, in contrast to the existing Cahn-Hilliard models.

\section{EXTENDED GRAND-POTENTIAL MODEL}

\section{A. Grand-potential model}

The grand-potential approach $[14,15]$ essentially makes a mark by preferring the pair (grand potential, chemical potential) over the much traditional combination [(Helmholtz) free energy, concentration]. The formulation begins by expressing the grand-potential functional of the system of volume $V$ as

$$
\Psi(\boldsymbol{\phi}, \nabla \boldsymbol{\phi}, \boldsymbol{\mu})=\int_{V} \frac{1}{\epsilon} w(\boldsymbol{\phi})+\epsilon a(\nabla \boldsymbol{\phi})+\psi(\boldsymbol{\phi}, \boldsymbol{\mu}) d V,
$$

which applies for a multiphase and multicomponent system of $N$ phases $\left(\phi \equiv\left\{\phi_{\alpha}, \ldots, \phi_{N}\right\}\right)$ and $K$ components with the corresponding chemical potentials $\boldsymbol{\mu} \equiv\left\{\mu_{1}, \ldots, \mu_{K-1}\right\}$. Here $w\left(\right.$ in $\mathrm{J} / \mathrm{m}^{2}$ ) and $a\left(\right.$ in $\mathrm{J} / \mathrm{m}^{4}$ ) are the potential and gradient contributions to the interfacial free energy and $\epsilon$ is a parameter related to the interface thickness (a length in $\mathrm{m}$ ). Using the dimensionless interpolation function $h_{\alpha}(\boldsymbol{\phi})$, the overall grandpotential density $\psi$ (of unit $\mathrm{J} / \mathrm{m}^{3}$ ) is expressed as

$$
\psi(\boldsymbol{\phi}, \boldsymbol{\mu})=\sum_{\alpha=1}^{N} \psi^{\alpha}(\boldsymbol{\mu}) h_{\alpha}(\boldsymbol{\phi}),
$$

where the grand-potential densities of the individual phases $\psi^{\alpha}$

$$
\psi^{\alpha}(\boldsymbol{\mu})=f^{\alpha}\left[\boldsymbol{c}^{\alpha}(\boldsymbol{\mu})\right]-\sum_{i=1}^{K-1} \mu_{i} c_{i}^{\alpha}(\boldsymbol{\mu}), \forall \alpha \in\{1, \ldots, N\}
$$

are expressed by the individual bulk free energies $f^{\alpha}$ (in $\mathrm{J} / \mathrm{m}^{3}$ ) and the phase-dependent compositions $\boldsymbol{c}^{\alpha} \equiv\left\{c_{1}^{\alpha}, \ldots, c_{K-1}^{\alpha}\right\}$ (mole fractions, dimensionless).

A quasi-equilibrium is locally assumed between the phases, such that

$$
\underbrace{\frac{\partial f^{\alpha}\left(\boldsymbol{c}^{\alpha}\right)}{\partial c_{i}^{\alpha}}}_{\equiv \mu_{i}^{\alpha}}=\underbrace{\frac{\partial f^{\beta}\left(\boldsymbol{c}^{\beta}\right)}{\partial c_{i}^{\beta}}}_{\equiv \mu_{i}^{\beta}}=\cdots=\mu_{i}, \quad \forall\{i, \alpha\} .
$$

When treating $c_{i}^{\alpha}$ as mole fractions, please note that the notion of a chemical potential in the phase-field model is slightly different from the standard thermodynamic definition. The 
quantities $\mu_{i}$ are of unit $\mathrm{J} / \mathrm{m}^{3}$ and hence are chemical potentials per unit volume. Since the molar volume $V_{m}$ is typically a constant in the grand-potential model, which is independent of the phase and the composition, the chemical potential is related to the true chemical potential $\tilde{\mu}_{i}$, of unit $\mathrm{J} / \mathrm{mol}$, by means of $\mu_{i}=\tilde{\mu}_{i} / V_{m}$. In the following, both symbols will be used. To maintain a certain level of consistency, whenever a chemical potential of unit $\mathrm{J} / \mathrm{mol}$ appears, it is written with a tilde, otherwise the unit $\mathrm{J} / \mathrm{m}^{3}$ does apply. Following [13,78], the chemical potentials should be treated more as chemical potential differences (so-called diffusion potentials), according to the definition above. Therefore, we write $\tilde{\mu}_{i} \equiv \tilde{\mu}_{i}^{\text {ind }}-\tilde{\mu}_{K}^{\text {ind }}$, where $\tilde{\mu}_{i}^{\text {ind }}$ represents the true individual chemical potential of the component $i$. This is also the reason why a number of $K-1$ chemical potentials suffice to completely specify the chemical state of the system.

From the phase-dependent $\boldsymbol{c}^{\alpha}$, an interpolated composition $\boldsymbol{c} \equiv\left\{c_{1}, \ldots, c_{K-1}\right\}$ is written as

$$
c_{i}=\sum_{\alpha=1}^{N} c_{i}^{\alpha} h_{\alpha}(\boldsymbol{\phi}), \quad \forall 1 \leqslant i \leqslant K-1,
$$

which may also be derived as a derivative of the grand potential $\partial \psi / \partial \mu_{i}=-c_{i}$. The composition of the $K$ th component is implicitly treated through the sum condition $\sum_{i=1}^{K} c_{i}=1$. Here the interpolation function $h_{\alpha}(\boldsymbol{\phi})$ is appropriately formulated to exhibit a smooth and monotonic transition across the diffuse interface. Additionally, it satisfies the conditions

$$
\begin{gathered}
\sum_{\alpha=1}^{N} h_{\alpha}(\boldsymbol{\phi})=1, \\
h_{\alpha}\left(\left\{\phi_{\alpha}=0, \ldots\right\}\right)=0 \forall \alpha, \\
h_{\alpha}\left(\left\{\phi_{\alpha}=1, \ldots\right\}\right)=1 \forall \alpha .
\end{gathered}
$$

The temporal evolution of the interpolated composition for the component $i$ can be expressed as

$$
\dot{c}_{i}=-\nabla \cdot \dot{j}_{i}
$$

which preserves its conserved nature, where $\boldsymbol{j}_{i}$ is the corresponding flux. Note that the flux is written here in terms of composition, instead of concentration, and hence is of unit $\mathrm{m} / \mathrm{s}$.

Rewriting Eq. (16) with the help of Eq. (12) and using the product rule

$$
\dot{c}_{i}=\sum_{\alpha=1}^{N} \dot{h}_{\alpha}(\boldsymbol{\phi}) c_{i}^{\alpha}(\boldsymbol{\mu})+\sum_{\alpha=1}^{N} h_{\alpha}(\boldsymbol{\phi}) \dot{c}_{i}^{\alpha}(\boldsymbol{\mu}),
$$

the evolution of the dynamic variable, the chemical potential, is obtained by inverting each $\dot{c}_{i}^{\alpha}(\boldsymbol{\mu})$, in favor of $\dot{\mu}$, as

$$
\dot{\mu}_{i}=\left[\sum_{\alpha=1}^{N} h_{\alpha}(\boldsymbol{\phi}) \frac{\partial c_{j}^{\alpha}(\boldsymbol{\mu})}{\partial \mu_{i}}\right]^{-1}\left[\dot{c}_{j}-\sum_{\alpha=1}^{N} c_{j}^{\alpha}(\boldsymbol{\mu}) \dot{h}_{\alpha}(\boldsymbol{\phi})\right] .
$$

Please note that for the sake of brevity, the above equation is written in the Einstein notation and represents a linear system of $K-1$ equations, in the most general case.

The grand potential above may be obtained through a Legendre transformation of the free-energy density of the individual phases, with respect to the composition from a related model [13]. However, the model from Eiken et al. requires to be solved for each of the phase-dependent compositions $c^{\alpha}$. The advantage of treating a single chemical potential, instead of $N$ phase-dependent compositions, is that the computational complexity is largely reduced. The reduction applied through Eqs. (17) and (18) rests on the explicit invertibility of the phase-dependent composition and the chemical potential, which can be written as $c_{i}^{\alpha}(\boldsymbol{\mu})$, for example. In the grand-potential model, the chemical potentials $\boldsymbol{\mu}$ therefore are identified as the fundamental variables which govern the evolution.

The phase fields in the grand-potential model are nonconserved, and their evolution is commonly expressed by a multiphase extension of the Allen-Cahn equation. In the present approach, the migration of the interface is formulated as the pairwise interaction of all possible phase combinations [17]. Correspondingly, the temporal evolution of the phase field is expressed as

$$
\epsilon \dot{\phi}_{\alpha}=\frac{1}{\tilde{N}} \sum_{\substack{\beta=1 \\ \beta \neq \alpha}}^{N} m_{\alpha \beta}\left(\frac{\delta \Psi}{\delta \phi_{\beta}}-\frac{\delta \Psi}{\delta \phi_{\alpha}}\right),
$$

where $m_{\alpha \beta}$ is the mobility of the interface (of unit $\mathrm{m}^{4} /(\mathrm{Js})$ ), separating the phases $\alpha$ and $\beta$, and $\tilde{N}$ denotes the number of locally coexisting phases. For an introduction to boundary mobilities, the interested reader is referred to [79, Chap. 5].

The grand-potential model is formulated both in conjunction with a well-type potential [14] and an obstacle-type potential [15]. In the current work, the obstacle-type potential is utilized, which is written as

$$
w(\boldsymbol{\phi})= \begin{cases}\frac{16}{\pi^{2}} \sum_{\substack{\alpha, \beta=1 \\ \alpha<\beta}}^{N, N} \gamma_{\alpha \beta} \phi_{\alpha} \phi_{\beta}, & \boldsymbol{\phi} \in \mathcal{G} \\ \infty, & \boldsymbol{\phi} \notin \mathcal{G},\end{cases}
$$

where a Gibbs simplex $\mathcal{G}$, which reads as

$$
\mathcal{G}=\left\{\sum_{\alpha=1}^{N} \phi_{\alpha}=1: \phi_{\alpha} \geqslant 0 \forall \alpha\right\},
$$

is added to the formulation. The simplex $\mathcal{G}$ heavily penalizes the phase field beyond the imposed condition. For the current multiphase-field treatment, the gradient energy density $a(\nabla \boldsymbol{\phi})$ is expressed as [17]

$$
a(\nabla \phi)=\sum_{\substack{\alpha, \beta=1 \\ \alpha<\beta}}^{N, N} \gamma_{\alpha \beta} \nabla \phi_{\alpha} \cdot \nabla \phi_{\beta},
$$

where $\gamma_{\alpha \beta}$ denotes the interfacial energy (of unit $\mathrm{J} / \mathrm{m}^{2}$ ) between the $\alpha$ and $\beta$ phase. This completes the model specification.

A few interesting features of the grand-potential model are worth noting. First, it elegantly avoids an artificial coupling between the interfacial and the bulk contributions, which usually arises in models taking the (free energy, concentration) pair route [80]. In contrast, alternate techniques avoid the coupling either by specific relations between the interpolation 
functions $[11,12]$ or by a so-called two-phase or mixture philosophy $[10,13]$.

The phase-field approach, based on the grand-potential formalism, has been employed extensively to model solidification [81-84] and solid-state transformation [85,86], including multicomponent systems [87,88]. Furthermore, this technique is also combined with an elastic model, in order to analyze chemoelastic transformations [89,90]. Much different from these conventional studies, this approach has recently been adopted to investigate energy-minimizing, curvaturedriven transformations, where the phase field behaves in a conserved fashion [91-95]. In all analyses involving the grand-potential formalism, volume diffusion is treated as the primary mode of mass transfer.

Moreover, it should be mentioned that the ability to recover the sharp interface solution is not discussed in works where surface and grain-boundary diffusion are introduced $[74,75,96]$. This is the starting point for the extension presented in the following section.

\section{B. Introducing surface flux}

In the grand-potential framework, the composition evolution is often formulated to exclusively include bulk diffusivities. Such considerations overlook the physically conceivable atomic migration along the local tangential directions of the interface. Therefore, the existing approach is extended in the present work, by explicitly including an interface flux in the composition evolution. Accordingly, the flux $\boldsymbol{j}_{i}$ in Eq. (16) is written as

$$
\boldsymbol{j}_{i}=\boldsymbol{j}_{i}^{b}+\boldsymbol{j}_{i}^{s},
$$

where $\boldsymbol{j}_{i}^{b}$ and $\boldsymbol{j}_{i}^{s}$ correspond to the flux contributed by volume (bulk) and surface diffusion, respectively. Analogous to the existing formulations, the bulk flux is expressed as

$$
\boldsymbol{j}_{i}^{b}=-\sum_{\alpha=1}^{N} \sum_{j=1}^{K-1} M_{i j}^{\alpha}(\boldsymbol{\mu}) h_{\alpha}(\boldsymbol{\phi}) \nabla \mu_{j} .
$$

Since the composition is generally expressed in mole (or site) fractions, the flux of the solvent is ascertained from the evolution of $K-1$ components. In Eq. (24), the mobility of the $i$ th migrating species is dictated by $M_{i j}^{\alpha}(\boldsymbol{\mu})=D_{i j}^{\alpha} \partial c_{i}^{\alpha}(\boldsymbol{\mu}) / \partial \mu_{j}$, which includes the interdiffusivity matrix $D_{i j}^{\alpha}$ and a susceptibility matrix $\partial c_{i}^{\alpha}(\boldsymbol{\mu}) / \partial \mu_{j}$.

The surface flux, included in the extended composition evolution in Eq. (16), is written as

$$
\boldsymbol{j}_{i}^{s}=-\frac{1}{\epsilon} \sum_{\substack{\alpha, \beta=1 \\ \alpha<\beta}}^{N, N} \sum_{j=1}^{K-1} M_{i j}^{\alpha \beta}(\boldsymbol{\phi}) \nabla \mu_{j},
$$

where the degenerate mobility matrix for a particular $\alpha-\beta$ interface $\boldsymbol{M}^{\alpha \beta}(\boldsymbol{\phi}) \in \mathbb{R}^{(K-1) \times(K-1)}$ writes as $\overline{\boldsymbol{M}}^{\alpha \beta} g_{\alpha \beta}(\boldsymbol{\phi})$. The prefactors $\overline{\boldsymbol{M}}^{\alpha \beta} \in \mathbb{R}^{(K-1) \times(K-1)}$, which quantify the surface diffusion and are of unit $\mathrm{m}^{6} /(\mathrm{J} \mathrm{s})$, can be treated as constant. However, when including the appropriate susceptibility parameters, they may be expressed quantitatively by an interdiffusivity matrix and a constant related to the activation energy. In Eq. (25), the inclusion of $\epsilon$ ensures that the transport along the interface will not vanish for small interface widths. The interfacial mobility function $g_{\alpha \beta}(\boldsymbol{\phi})$ is unitless and appears as a bell-like function to satisfy the condition

$$
g_{\alpha \beta}(\phi)= \begin{cases}>0, & \phi_{\alpha}>0 \cap \phi_{\beta}>0 \\ 0, & \phi_{\alpha}=0 \cup \phi_{\beta}=0 .\end{cases}
$$

Numerous forms of $g_{\alpha \beta}(\phi)$ have hitherto been involved to confine the diffusion to the interface. Examples which satisfy the condition stated above are $g_{\alpha \beta}(\boldsymbol{\phi})=\phi_{\alpha}^{n} \phi_{\beta}^{n}$, with $n>0$, $n \in \mathbb{R}$.

\section{SHARP-INTERFACE RELATIONS}

The effect of curvature on the chemical potential is given by [97]

$$
\tilde{\mu}=\tilde{\mu}_{\mathrm{eq}}+V_{m} \kappa_{s} \gamma_{s}
$$

where $\mu_{\text {eq }}$ represents the chemical potential in equilibrium with a flat surface, $V_{m}$ denotes the molar volume, $\gamma_{s}$ represents the (isotropic) interfacial energy of the surface, and $\kappa_{s}$ denotes the signed curvature of the surface. Given that a two-dimensional surface profile is represented as $y(x, t)$ in a Cartesian coordinate system, at time $t$, the curvature writes as

$$
\kappa_{s}=-\frac{y^{\prime \prime}}{\left(1+\left(y^{\prime}\right)^{2}\right)^{3 / 2}},
$$

where primes denote differentiation with respect to $x$.

Please note that a common misconception is to assume Eq. (27) to be universally valid, which is emphasized in [98, p. 434]. A more generic and rigorous relation can be given, which is shown in Appendix A. However, the above equation is the basis of the historically grown surface diffusion theory, which is presented in the following subsection.

\section{A. Surface diffusion}

According to Mullins [19], as a result of surface diffusion, matter is tangentially transported to the interface, with a flux of

$$
\boldsymbol{j}_{s}=-\frac{D_{s} \rho_{s}}{R T} \nabla_{s} \tilde{\mu},
$$

where $D_{s}$ denotes the surface diffusion coefficient (in $\mathrm{m}^{2} / \mathrm{s}$ ), $\rho_{s}$ represents the number of atoms per surface area (in $\left.\mathrm{mol} / \mathrm{m}^{2}\right), R$ is the ideal gas constant $[\approx 8.314 \mathrm{~J} /(\mathrm{mol} \mathrm{K})], T$ denotes the temperature (in $\mathrm{K}$ ), and $\nabla_{s}$ represents the surface gradient operator. The surface flux therefore is of unit $\mathrm{mol} /(\mathrm{ms})$, and hence is not a usual flux, but the surface excess flux. For a definition of the surface excess properties, the interested reader is referred to [99, p. $7 \mathrm{ff} .$, p. $55 \mathrm{ff}$.] and [100, p. $179 \mathrm{ff}$.$] . The surface divergence \nabla_{s}$. of the surface excess flux leads to a normal displacement of the surface profile, which can be written as

$$
v_{n}=-V_{m} \nabla_{s} \cdot \boldsymbol{j}_{s}=\frac{D_{s} V_{m} \rho_{s}}{R T} \nabla_{s}^{2} \tilde{\mu},
$$

where $v_{n}$ denotes the scalar normal velocity of the interface and $\nabla_{s}^{2}$ represents the surface Laplacian operator. By inserting the relation (27), the above equation is expressed in terms of 
curvature to obtain

$$
v_{n}=\frac{D_{s} V_{m}^{2} \rho_{s} \gamma_{s}}{R T} \nabla_{s}^{2} \kappa_{s}=B \nabla_{s}^{2} \kappa_{s}
$$

as the governing equation for a change in the shape of the surface. Thus, the rate at which the surface evolves is controlled by the rate constant $B$ (of unit $\mathrm{m}^{4} / \mathrm{s}$ ), which is defined as

$$
B \equiv \frac{D_{s} V_{m}^{2} \rho_{s} \gamma_{s}}{R T} .
$$

\section{B. Surface diffusion and attachment kinetics}

While exclusively considering surface diffusion, the process of incorporating atoms at the surface is (tacitly) assumed to be comparatively fast. However, the rate at which atoms can be attached to or detached from the surface is limited, since the corresponding material property is the mobility of the interface $m_{s}$ (of unit $\mathrm{m}^{4} /(\mathrm{J} \mathrm{s})$ ). This property reflects the ease of attaching or detaching atoms from the surface. In the work of Cahn et al. [21], a modified governing law is derived for the combination of surface diffusion and attachment kinetics. The corresponding equation in the current notation writes as

$$
v_{n}=-\nabla_{s}^{2}\left(\frac{1}{m_{s}} \nabla_{s}^{2}-\frac{\gamma_{s}}{B}\right)^{-1} \kappa_{s} \gamma_{s}
$$

Interestingly, the above equation introduces another length scale:

$$
l_{c} \equiv \sqrt{\frac{B}{\gamma_{s} m_{s}}}=\sqrt{\frac{D_{s} V_{m}^{2} \rho_{s}}{R T m_{s}}},
$$

while the motion by attachment kinetics dominates at length scales much smaller than this quantity. In the limit of a highly mobile boundary $\left(m_{s} \rightarrow \infty\right)$, the length scale at which the kinetics are dominated by the attachment becomes negligibly small $\left(l_{c} \rightarrow 0\right)$ and the same law as given in Eq. (31) is recovered. For fast diffusion $(B \rightarrow \infty)$, the motion caused by the difference of the mean curvature dominates at all length scales $\left(l_{c} \rightarrow \infty\right)$. According to [32], Eq. (33) can be rewritten as a system of two coupled second-order equations:

$$
\begin{gathered}
v_{n}=\frac{B}{\gamma_{s}} \nabla_{s}^{2} \mu, \\
\mu=\mu_{\mathrm{eq}}+\kappa_{s} \gamma_{s}+\frac{v_{n}}{m_{s}} .
\end{gathered}
$$

Therefore, the presence of limited attachment kinetics results in a relaxed form of the Gibbs-Thomson relation, including a term which is proportional to the interface velocity.

\section{ASYMPTOTIC ANALYSIS}

The ability of a phase-field technique to recover the sharpinterface solution is commonly elucidated through asymptotic analysis $[9,11,15,22,31,32,45,47,59,60,101]$. Moreover, to calibrate the model, the asymptotics serves as a method to identify the (otherwise unknown) relation between material properties and model parameters. Therefore, in performing this analysis, a quantitative physical interpretation of the simulation results is achieved. The original extension of the grand potential approach to a multiphase system includes asymptotic treatment [15]. Since these asymptotics were primarily directed toward solidification, vanishing diffusivity, at one side of the interface, accompanies the analysis. Despite the subsequent extensions [93,102], the consideration of volume diffusion as the only mode of mass transfer reflects in the asymptotics. Therefore, the present model is asymptotically treated for an exclusively surface-diffusion governed evolution. Since the cumulative roles of surface and volume diffusion are formulated linearly, the exclusive consideration of surface diffusion in the asymptotics is considered reasonable. Furthermore, owing to the significance of surface diffusion in curvature-driven transformations, the following asymptotics is delineated correspondingly.

\section{A. Evolution equations for a two-phase system}

Although the model is formulated for multiphase and multicomponent systems, the evolution of a two-phase binary system is analyzed asymptotically. The two-phase consideration obviates the need for a vector representation of the phase field, and a sole order parameter $\phi_{\alpha}$ remains, while the second phase $\phi_{\beta}=1-\phi_{\alpha}$ is considered implicitly. Additionally, the single independent composition and the chemical potential are denoted by $c \equiv c_{1}$ and $\mu \equiv \mu_{1}$, without any subscript. Moreover, since the surface diffusion is assumed to be the exclusive path for the mass transfer, the corresponding mobility is represented by $M\left(\phi_{\alpha}\right)=\bar{M}^{\alpha \beta} g\left(\phi_{\alpha}\right) \equiv M_{11}^{\alpha \beta}(\phi)$.

From Eqs. (19) and (18), the evolution equations for the two-phase binary systems are expressed as

$$
\begin{aligned}
\frac{\epsilon}{m_{\alpha \beta}} \dot{\phi}_{\alpha} & =\epsilon \gamma_{\alpha \beta} \nabla^{2} \phi_{\alpha}-\frac{8 \gamma_{\alpha \beta}}{\epsilon \pi^{2}}\left(1-2 \phi_{\alpha}\right)-\frac{\psi_{\alpha}-\psi_{\beta}}{2} \frac{\partial h_{\alpha}}{\partial \phi_{\alpha}}, \\
\frac{\partial c}{\partial \mu} \dot{\mu} & =\nabla \cdot \frac{M\left(\phi_{\alpha}\right)}{\epsilon} \nabla \mu-\left(c^{\alpha}(\mu)-c^{\beta}(\mu)\right) \dot{h}_{\alpha} .
\end{aligned}
$$

While the first term on the right-hand side of Eq. (37) is the derivative of the gradient-energy function, the obstacle-type potential in the present formulation yields the second term. The operation of the well-type potential is fundamentally different from the obstacle-type potential. While in well-type functions, the bulk values of the phase fields are achieved trough their global minima, the piecewise nature in obstacletype potentials is responsible for the boundedness of the phase field. For a well potential-type equation, the domain is commonly split into inner and outer regions, and solutions are obtained by matching both of them. However, due to the strictly finite width of the interface, resulting from the obstacle potential, and owing to the sole consideration of surface diffusion, a procedure following Cahn et al. [22] is adopted. Accordingly, the current analysis is restricted to the inner region, where the phase field remains strictly bounded between zero and unity.

\section{B. Curvilinear coordinates}

The governing equations may be expressed in a moving curvilinear coordinate system, which is parameterized by $(r, s, t)$, where $r$ denotes the signed distance from the isosurface $r=0$ and $s$ measures the arc length along it, at time $t$. Then the position $\boldsymbol{x}$ can be written as $\boldsymbol{x}(r, s, t)=$ $\boldsymbol{x}_{0}(s, t)+\boldsymbol{r n}(s, t)$, where $\boldsymbol{x}_{0}(s, t)$ is referred to as the baseline. 
The normal vector $\boldsymbol{n}$ and the tangential vector $\boldsymbol{s} \equiv \partial \boldsymbol{x}_{0} / \partial s$ are mutually orthonormal vectors, respectively pointing into the direction of increasing $r$ and $s$. The origin of the normal variable $r$ is chosen in such a way that $r=0$ corresponds to the isosurface $\phi_{\alpha}=1 / 2$. Note that by this choice, the curvature $\kappa_{0}$ of the baseline $r=0$ corresponds to the curvature of the isosurface $\phi_{\alpha}=1 / 2$, and hence is a property of the phase field. In the current paper, a sign convention for curvature is chosen such that in the case of a positive sign of $\kappa_{0}, \boldsymbol{n}$ points from the center of curvature to a point on the baseline. The scalar normal velocity $v_{n} \equiv \boldsymbol{n} \cdot \partial \boldsymbol{x} /\left.\partial t\right|_{r, s}$ delineates the motion of this phase-field contour in space. Since $r$ is the signed distance from the baseline, $v_{n}$ is independent on $r$. Let us denote the positions $r_{+}$and $r_{-}$as the points where the phase field assumes zero and unity values, respectively. Then its minimum and maximum values are assumed at these points, according to the Gibbs simplex [Eq. (21)]. Thus, the following conditions are satisfied:

$$
\begin{aligned}
\phi_{\alpha}(r=0, s) & =1 / 2, \\
\phi_{\alpha}\left(r_{-}, s\right) & =1, \\
\phi_{\alpha}\left(r_{+}, s\right) & =0, \\
\left.\frac{\partial \phi_{\alpha}}{\partial r}\right|_{r_{ \pm}, s} & =0 .
\end{aligned}
$$

While the behavior of the phase field inside the interface is well characterized by Eq. (37), the above conditions are required to capture the inherent behavior of the solutions, as the bulk is approached, where $r>r_{+} \cup r<r_{-}$. For the diffusion equation, a flux boundary condition is accordingly applied at either end of the interface. The corresponding relations read as

$$
\lim _{r \rightarrow r_{ \pm}}-\frac{M\left(\phi_{\alpha}\right)}{\epsilon} \frac{\partial \mu}{\partial r}=v_{n}\left[c\left(r_{ \pm}\right)-c^{ \pm}\right],
$$

where $c\left(r_{ \pm}\right)$is the interpolated composition as an inner limit (from within the interface) and $c^{ \pm}$is the corresponding outer limit (from the bulk). The above relation is derived from a balance at the interfacial endpoints, as shown in Appendix C 2.

Now the inner region, which is also referred to as the interface, is well defined as the interval $r_{-} \leqslant r \leqslant r_{+}$. The endpoints $r_{ \pm}$are not known a priori, but their derivation is part of the problem. In the curvilinear coordinate system, the differential operators are expressed as

$$
\begin{gathered}
\nabla=n \frac{\partial}{\partial r}+s \frac{1}{1+r \kappa_{0}} \frac{\partial}{\partial s}, \\
\nabla \cdot(a \boldsymbol{\nabla} b)=\frac{\partial}{\partial r}\left(a \frac{\partial b}{\partial r}\right)+\frac{1}{\left(1+r \kappa_{0}\right)^{2}} \frac{\partial}{\partial s}\left(a \frac{\partial b}{\partial s}\right) \\
+a\left[\frac{\kappa_{0}}{1+r \kappa_{0}} \frac{\partial b}{\partial r}-\frac{r \frac{\partial \kappa_{0}}{\partial s}}{\left(1+r \kappa_{0}\right)^{3}} \frac{\partial b}{\partial s}\right], \\
\nabla^{2}=\frac{\partial^{2}}{\partial r^{2}}+\frac{\kappa_{0}}{1+r \kappa_{0}} \frac{\partial}{\partial r}+\frac{1}{\left(1+r \kappa_{0}\right)^{2}} \frac{\partial^{2}}{\partial s^{2}}-\frac{r \frac{\partial \kappa_{0}}{\partial s}}{\left(1+r \kappa_{0}\right)^{3}} \frac{\partial}{\partial s},
\end{gathered}
$$

which can be derived from differential geometry, as given in [103].

\section{Preliminary statements}

In the subsequent analysis, the set of equations will be evaluated order by order. In order to simplify the analysis, frequently used formulas and symbols are stated in the following.

To study the inner region, a stretched coordinate $\eta=r / \epsilon$ is utilized. Rescaled and expanded in powers of $\epsilon$, one obtains

$$
\begin{gathered}
\nabla=\frac{1}{\epsilon} \boldsymbol{n} \frac{\partial}{\partial \eta}+s \frac{\partial}{\partial s}+O(\epsilon), \\
\nabla \cdot(a \nabla b)=\frac{1}{\epsilon^{2}} \frac{\partial}{\partial \eta}\left(a \frac{\partial b}{\partial \eta}\right)+\frac{a \kappa_{0}}{\epsilon} \frac{\partial b}{\partial \eta} \\
-a \eta \kappa_{0}^{2} \frac{\partial b}{\partial \eta}+\frac{\partial}{\partial s}\left(a \frac{\partial b}{\partial s}\right)+O(\epsilon), \\
\nabla^{2}=\frac{1}{\epsilon^{2}} \frac{\partial^{2}}{\partial \eta^{2}}+\frac{\kappa_{0}}{\epsilon} \frac{\partial}{\partial \eta}-\eta \kappa_{0}^{2} \frac{\partial}{\partial \eta}+\frac{\partial^{2}}{\partial s^{2}}+O(\epsilon) .
\end{gathered}
$$

We may express the temporal derivative $\partial \varphi /\left.\partial t\right|_{r, s, t}$ of any scalar field $\varphi(\boldsymbol{x}, t)$, observed by following the trajectory of the point $\boldsymbol{x}(r, s, t)$ on the surface at constant $r$ and $s$, as [104, cf. Eq. (3.7)]

$$
\left.\frac{\partial \varphi(\boldsymbol{x}(r, s, t), t)}{\partial t}\right|_{r, s, t}=\left.\nabla \varphi \cdot \frac{\partial \boldsymbol{x}}{\partial t}\right|_{r, s, t}+\left.\frac{\partial \varphi}{\partial t}\right|_{\boldsymbol{x}(r, s, t)} .
$$

The last term is the standard time derivative of the scalar field holding $\boldsymbol{x}$ fixed $\left(\partial \varphi /\left.\partial t\right|_{\boldsymbol{x}} \equiv \dot{\varphi}\right) . \boldsymbol{v}(r, s, t) \equiv \partial \boldsymbol{x} /\left.\partial t\right|_{r, s, t}$ denotes the parametrization-dependent surface velocity, which tracks the motion of the surface, keeping $r$ and $s$ fixed. Since $\nabla \varphi=$ $\nabla_{s} \varphi+\boldsymbol{n} \partial \varphi / \partial r$ and $\boldsymbol{v}=\boldsymbol{v}_{\tan }+\boldsymbol{n} v_{n}$, where $\boldsymbol{v}_{\tan }$ denotes the parametrization-dependent tangential velocity, one obtains

$$
\dot{\varphi}=\left.\frac{\partial \varphi}{\partial t}\right|_{r, s, t}-\boldsymbol{v}_{\tan } \cdot \nabla_{s} \varphi-v_{n} \frac{\partial \varphi}{\partial r} .
$$

Note that $\stackrel{\circ}{\rho} \equiv \partial \varphi /\left.\partial t\right|_{r, s, t}-\boldsymbol{v}_{\tan } \cdot \nabla_{s} \varphi$ is the parametrizationindependent normal time derivative [104, Eq. (3.12)], which provides the temporal change of the variable following the normal trajectory of the surface. Hence it follows that

$$
\dot{\varphi}=\stackrel{\circ}{\varphi}-v_{n} \frac{\partial \varphi}{\partial r} .
$$

When applied to the phase field and the chemical potential, this results in

$$
\begin{gathered}
\dot{\phi}_{\alpha}=\stackrel{\circ}{\phi}_{\alpha}-\frac{v_{n}}{\epsilon} \frac{\partial \phi_{\alpha}}{\partial \eta}, \\
\dot{\mu}=\stackrel{\leftrightarrow}{\mu}-\frac{v_{n}}{\epsilon} \frac{\partial \mu}{\partial \eta} .
\end{gathered}
$$

The width of the interface in the stretched coordinate system is denoted by $\delta$ and is defined as $\delta \equiv \eta_{+}-\eta_{-}$, where $\eta_{ \pm}$ denotes the position of the ends of the inner region $\eta_{ \pm}=r_{ \pm} / \epsilon$. The phase field and chemical potential, expanded in powers of $\epsilon$, write as

$$
\begin{gathered}
\phi_{\alpha}=\phi_{\alpha}^{0}+\epsilon \phi_{\alpha}^{1}+\epsilon^{2} \phi_{\alpha}^{2}+O\left(\epsilon^{3}\right), \\
\mu=\mu^{0}+\epsilon \mu^{1}+\epsilon^{2} \mu^{2}+O\left(\epsilon^{3}\right) .
\end{gathered}
$$


Note that both $\eta_{ \pm}$are not necessarily independent of $\epsilon$, but may change due to the presence of $\phi_{\alpha}^{1}$ and higher-order contributions. Therefore, both endpoints are expressed as

$$
\eta_{ \pm}=\eta_{ \pm}^{0}+\epsilon \eta_{ \pm}^{1}+\epsilon^{2} \eta_{ \pm}^{2}+O\left(\epsilon^{3}\right)
$$

The two quantities $M$ and $h_{\alpha}$ are only a function of the order parameter:

$$
\begin{gathered}
M=M^{0}+\epsilon M^{1}+\epsilon^{2} M^{2}+O\left(\epsilon^{3}\right), \\
h_{\alpha}=h_{\alpha}^{0}+\epsilon h_{\alpha}^{1}+\epsilon^{2} h_{\alpha}^{2}+O\left(\epsilon^{3}\right) .
\end{gathered}
$$

Therefore, the lowest order terms write as $\left.h_{\alpha}^{0} \equiv h_{\alpha}\right|_{\epsilon=0}=$ $h_{\alpha}\left(\phi_{\alpha}^{0}\right)$ and $\left.M^{0} \equiv M\right|_{\epsilon=0}=M\left(\phi_{\alpha}^{0}\right)$. Bear in mind that $\dot{h}_{\alpha}=$ $\stackrel{\circ}{h}_{\alpha}-v_{n} / \epsilon \partial h_{\alpha} / \partial \eta$. To incorporate the boundary conditions [Eqs. (42) and (43)] in the rescaled coordinate system order by order, Taylor expansions are performed in Appendix C. It will be useful to introduce the quantity $J \equiv M \partial \mu / \partial \eta$, which represents the negative rescaled flux in the normal direction, and rewrite it in the following form:

$$
J=J^{0}+\epsilon J^{1}+\epsilon^{2} J^{2}+O\left(\epsilon^{3}\right),
$$

where the individual terms necessarily take the form

$$
\begin{gathered}
J^{0} \equiv M^{0} \frac{\partial \mu^{0}}{\partial \eta} \\
J^{1} \equiv M^{0} \frac{\partial \mu^{1}}{\partial \eta}+M^{1} \frac{\partial \mu^{0}}{\partial \eta} \\
J^{2} \equiv M^{0} \frac{\partial \mu^{2}}{\partial \eta}+M^{1} \frac{\partial \mu^{1}}{\partial \eta}+M^{2} \frac{\partial \mu^{0}}{\partial \eta}
\end{gathered}
$$

By expressing the operators in terms of the stretched curvilinear coordinate system, the following evolution equation is obtained for the phase field:

$$
\begin{gathered}
\left(\frac{-v_{n}^{0}-\epsilon v_{n}^{1}}{m_{\alpha \beta} \gamma_{\alpha \beta}}-\kappa_{0}\right)\left(\frac{\partial \phi_{\alpha}^{0}}{\partial \eta}+\epsilon \frac{\partial \phi_{\alpha}^{1}}{\partial \eta}\right)+\epsilon \eta \kappa_{0}^{2} \frac{\partial \phi_{\alpha}^{0}}{\partial \eta}+\epsilon \dot{\phi}_{\alpha}^{0} \\
=\frac{1}{\epsilon} \frac{\partial^{2} \phi_{\alpha}^{0}}{\partial \eta^{2}}+\frac{\partial^{2} \phi_{\alpha}^{1}}{\partial \eta^{2}}+\epsilon \frac{\partial^{2} \phi_{\alpha}^{2}}{\partial \eta^{2}}+\epsilon \frac{\partial^{2} \phi_{\alpha}^{0}}{\partial s^{2}} \\
-\frac{8}{\pi^{2}}\left[\frac{1}{\epsilon}-2\left(\frac{1}{\epsilon} \phi_{\alpha}^{0}+\phi_{\alpha}^{1}+\epsilon \phi_{\alpha}^{2}\right)\right] \\
-\frac{\psi_{\alpha}\left(\mu^{0}\right)-\psi_{\beta}\left(\mu^{0}\right)}{2 \gamma_{\alpha \beta}}\left(\left.\frac{\partial h_{\alpha}}{\partial \phi_{\alpha}}\right|_{\epsilon=0}+\left.\epsilon \frac{\partial \frac{\partial h_{\alpha}}{\partial \phi_{\alpha}}}{\partial \epsilon}\right|_{\epsilon=0}\right) \\
-\left.\epsilon \mu^{1} \frac{\partial h_{\alpha}}{\partial \phi_{\alpha}}\right|_{\epsilon=0} \frac{\left.\frac{\partial \psi_{\alpha}}{\partial \mu}\right|_{\mu^{0}}-\left.\frac{\partial \psi_{\beta}}{\partial \mu}\right|_{\mu^{0}}}{2 \gamma_{\alpha \beta}}+O\left(\epsilon^{2}\right) .
\end{gathered}
$$

In the same stretched curvilinear coordinates, the evolution equation of the chemical potential writes as

$$
\begin{gathered}
\frac{1}{\epsilon^{3}} \frac{\partial}{\partial \eta}\left[\left(M^{0}+\epsilon M^{1}+\epsilon^{2} M^{2}\right) \frac{\partial\left(\mu^{0}+\epsilon \mu^{1}+\epsilon^{2} \mu^{2}\right)}{\partial \eta}\right] \\
+\frac{M^{0} \kappa_{0}}{\epsilon^{2}} \frac{\partial \mu^{0}}{\partial \eta}+\frac{M^{0} \kappa_{0}}{\epsilon} \frac{\partial \mu^{1}}{\partial \eta}+\frac{1}{\epsilon} \frac{\partial}{\partial s}\left(M^{0} \frac{\partial \mu^{0}}{\partial s}\right) \\
+\frac{M^{1} \kappa_{0}-M^{0} \eta \kappa_{0}^{2}+v_{n}^{0} \partial c /\left.\partial \mu\right|_{\phi_{\alpha}^{0}, \mu^{0}}}{\epsilon} \frac{\partial \mu^{0}}{\partial \eta} \\
+\left(c^{\alpha}\left(\mu^{0}\right)-c^{\beta}\left(\mu^{0}\right)\right) \frac{v_{n}}{\epsilon} \frac{\partial h_{\alpha}^{0}}{\partial \eta}+O(1)=0 .
\end{gathered}
$$

\section{Lowest order}

\section{Phase-field evolution}

The lowest order in the extended equation [Eq. (64)] pertains to $\epsilon^{-1}$. The terms associated with this order of $\epsilon$ are expressed as

$$
\frac{\partial^{2} \phi_{\alpha}^{0}}{\partial \eta^{2}}+\frac{16}{\pi^{2}} \phi_{\alpha}^{0}=\frac{8}{\pi^{2}} .
$$

The phase-field profile along the normal direction is obtained by starting with the general solution of the differential equation above, which reads as

$$
\phi_{\alpha}^{0}=\frac{1}{2}+C_{1} \cos \left(\frac{4}{\pi} \eta\right)+C_{2} \sin \left(\frac{4}{\pi} \eta\right),
$$

where $C_{1}$ and $C_{2}$ are two undetermined constants that are fixed by incorporating the corresponding boundary conditions. The first parameter $C_{1}=0$ is found by the relation $\phi_{\alpha}(\eta=0)=$ $1 / 2$, at the lowest order. The boundary conditions of the phase field, obtained from the corresponding expansions at the lowest order [Appendix C, Eq. (C5)], are $\phi_{\alpha}\left(\eta_{+}^{0}\right)=0$, $\phi_{\alpha}\left(\eta_{-}^{0}\right)=1$ and $\partial \phi_{\alpha} /\left.\partial \eta\right|_{\eta_{ \pm}^{0}}=0$. Subjecting the differential equation to these conditions results in the fact that not only the second unknown constant is fixed as $C_{2}=-1 / 2$, but also the initially unknown interfacial endpoints are found to be $\eta_{ \pm}^{0}= \pm \pi^{2} / 8$.

Therefore, the zeroth-order phase-field profile is well defined as

$$
\phi_{\alpha}^{0}=\frac{1}{2}-\frac{1}{2} \sin \left(\frac{4}{\pi} \eta\right) .
$$

There are two interesting properties of the solution that are worth considering: First, note that $\partial \phi_{\alpha}^{0} / \partial s=0$, which implies that $\partial h^{0} / \partial s \equiv \partial h_{\alpha}\left(\phi_{\alpha}^{0}\right) / \partial s=0$ and $\partial M^{0} / \partial s \equiv \partial M\left(\phi_{\alpha}^{0}\right) / \partial s=$ 0 . Additionally, it has to be noted that differentiating $\phi_{\alpha}^{0}$ with time, at constant $\eta$ and $s$, results in $\partial \phi_{\alpha}^{0} /\left.\partial t\right|_{r, s, t}=$ $\partial \phi_{\alpha}^{0} /\left.\partial t\right|_{\eta, s, t}=0$. Together with $\partial \phi_{\alpha}^{0} / \partial s=0$, it can be concluded that its normal time derivative $\dot{\phi}_{\alpha}^{0}$ vanishes.

\section{Chemical potential}

In the evolution equation of the chemical potential (65), the lowest-order terms correspond to $\epsilon^{-3}$. Only a single term 
appears at this order, which reads as

$$
\frac{\partial}{\partial \eta}\left[M\left(\phi_{\alpha}^{0}\right) \frac{\partial \mu^{0}}{\partial \eta}\right]=0
$$

Integration yields $M\left(\phi_{\alpha}^{0}\right) \partial \mu^{0} / \partial \eta=J^{0}(\eta)=C$, where $C$ is an undetermined constant. From the flux boundary condition at the lowest order (Appendix C 2), it follows that $J^{0}\left(\eta_{ \pm}^{0}\right)=0$. Therefore, the normal flux at the lowest order $\left[J^{0}(\eta)=M\left(\phi_{\alpha}^{0}\right) \partial \mu^{0} / \partial \eta=0\right]$ vanishes. According to Eq. (26) $M\left(\phi_{\alpha}^{0}\right)>0$ inside the interface. Hence $\mu^{0}$ assumes a constant value which needs to be fixed by a further investigation at higher orders.

\section{E. Next to lowest order \\ 1. Phase-field evolution}

At the next order, in Eq. (64), the terms appearing at the order unity are collected in the following equation:

$$
\begin{aligned}
\frac{\partial^{2} \phi_{\alpha}^{1}}{\partial \eta^{2}}+\frac{16}{\pi^{2}} \phi_{\alpha}^{1}= & \left(\frac{-v_{n}^{0}}{m_{\alpha \beta} \gamma_{\alpha \beta}}-\kappa_{0}\right) \frac{\partial \phi_{\alpha}^{0}}{\partial \eta} \\
& +\frac{\psi_{\alpha}\left(\mu^{0}\right)-\psi_{\beta}\left(\mu^{0}\right)}{2 \gamma_{\alpha \beta}} \frac{\partial h_{\alpha}\left(\phi_{\alpha}^{0}\right)}{\partial \phi_{\alpha}} .
\end{aligned}
$$

This is the lowest order at which curvature and a driving force, arising from the difference in grand potentials, appear, which govern the evolution. In order to obtain a relation between these two driving forces, the above equation is first multiplied on both sides by $\partial \phi_{\alpha}^{0} / \partial \eta$, to yield

$$
\begin{aligned}
& \frac{\partial^{2} \phi_{\alpha}^{1}}{\partial \eta^{2}} \frac{\partial \phi_{\alpha}^{0}}{\partial \eta}+\frac{16}{\pi^{2}} \phi_{\alpha}^{1} \frac{\partial \phi_{\alpha}^{0}}{\partial \eta} \\
& =\left(\frac{-v_{n}^{0}}{m_{\alpha \beta} \gamma_{\alpha \beta}}-\kappa_{0}\right)\left(\frac{\partial \phi_{\alpha}^{0}}{\partial \eta}\right)^{2} \\
& \quad+\frac{\psi_{\alpha}\left(\mu^{0}\right)-\psi_{\beta}\left(\mu^{0}\right)}{2 \gamma_{\alpha \beta}} \frac{\partial h_{\alpha}\left(\phi_{\alpha}^{0}\right)}{\partial \phi_{\alpha}} \frac{\partial \phi_{\alpha}^{0}}{\partial \eta} .
\end{aligned}
$$

Furthermore, to eliminate any dependence on $\eta$, the equation is subsequently integrated across the interface:

$$
\begin{aligned}
& \int_{\eta_{-}^{0}}^{\eta_{+}^{0}} \frac{\partial^{2} \phi_{\alpha}^{1}}{\partial \eta^{2}} \frac{\partial \phi_{\alpha}^{0}}{\partial \eta} d \eta+\frac{16}{\pi^{2}} \int_{\eta_{-}^{0}}^{\eta_{+}^{0}} \phi_{\alpha}^{1} \frac{\partial \phi_{\alpha}^{0}}{\partial \eta} d \eta \\
& =\left(\frac{-v_{n}^{0}}{m_{\alpha \beta} \gamma_{\alpha \beta}}-\kappa_{0}\right) \underbrace{\int_{\eta_{-}^{0}}^{\eta_{+}^{0}}\left(\frac{\partial \phi_{\alpha}^{0}}{\partial \eta}\right)^{2} d \eta}_{1 / 2} \\
& +\frac{\psi_{\alpha}\left(\mu^{0}\right)-\psi_{\beta}\left(\mu^{0}\right)}{2 \gamma_{\alpha \beta}} \int_{\eta_{-}^{0}}^{\eta_{+}^{0}} \frac{\partial h_{\alpha}\left(\phi_{\alpha}^{0}\right)}{\partial \phi_{\alpha}} \frac{\partial \phi_{\alpha}^{0}}{\partial \eta} d \eta .
\end{aligned}
$$

Evaluating the equation term by term shows that the last integral on the right-hand side appears to be the interpolation function and within the given interval identically is -1 , owing to its nature [Eqs. (14) and (15)]. The remaining contribution of the left-hand side is ascertained through two subsequent integrations by parts of the first term

$$
\begin{aligned}
& {\left[\frac{\partial \phi_{\alpha}^{1}}{\partial \eta} \frac{\partial \phi_{\alpha}^{0}}{\partial \eta}\right]_{\eta_{-}^{0}}^{\eta_{+}^{0}}-\left[\phi_{\alpha}^{1} \frac{\partial^{2} \phi_{\alpha}^{0}}{\partial \eta^{2}}\right]_{\eta_{-}^{0}}^{\eta_{+}^{0}}} \\
& \quad+\int_{\eta_{-}^{0}}^{\eta_{+}^{0}} \phi_{\alpha}^{1} \frac{\partial^{3} \phi_{\alpha}^{0}}{\partial \eta^{3}} d \eta+\frac{16}{\pi^{2}} \int_{\eta_{-}^{0}}^{\eta_{+}^{0}} \phi_{\alpha}^{1} \frac{\partial \phi_{\alpha}^{0}}{\partial \eta} d \eta \\
& =\frac{1}{2}\left(\frac{-v_{n}^{0}}{m_{\alpha \beta} \gamma_{\alpha \beta}}-\kappa_{0}\right)-\frac{\psi_{\alpha}\left(\mu^{0}\right)-\psi_{\beta}\left(\mu^{0}\right)}{2 \gamma_{\alpha \beta}} .
\end{aligned}
$$

The first term in brackets on the left-hand side turns out to vanish since $\partial \phi_{\alpha}^{0} /\left.\partial \eta\right|_{\eta_{ \pm}^{0}}=0$, if we, strictly speaking, assume the boundedness of $\partial \phi_{\alpha}^{1} /\left.\partial \eta\right|_{\eta_{ \pm}^{0}}$. To tighten the argument, this property of $\phi_{\alpha}^{1}$ is proven by analyzing its corresponding differential equation in Appendix D. According to Eq. (66) it follows $\partial^{3} \phi_{\alpha}^{0} / \partial \eta^{3}=-16 / \pi^{2} \partial \phi_{\alpha}^{0} / \partial \eta$, and thus the two integrals on the left-hand side cancel each other. The remaining terms read as

$$
-\left[\phi_{\alpha}^{1} \frac{\partial^{2} \phi_{\alpha}^{0}}{\partial \eta^{2}}\right]_{\eta_{-}^{0}}^{\eta_{+}^{0}}=\frac{1}{2}\left(\frac{-v_{n}^{0}}{m_{\alpha \beta} \gamma_{\alpha \beta}}-\kappa_{0}\right)-\frac{\psi_{\alpha}\left(\mu^{0}\right)-\psi_{\beta}\left(\mu^{0}\right)}{2 \gamma_{\alpha \beta}}
$$

In the case of a well potential, the ends of the inner region $\eta_{ \pm}$are $\pm \infty$, respectively, and the remaining bracketed term vanishes, due to the matching conditions, which require $\phi_{\alpha}^{1}$ to assume a value of zero at the ends. Choudhury et al. [15] treated the obstacle case in the same manner, while tacitly assuming that $\phi_{\alpha}^{1}$ has to vanish at the endpoints. However, no argument is given that provides a condition to validate this assumption. Due to the different treatment in the current work, the boundary conditions from Eqs. (40) and (41) are utilized to evaluate the nature of the bracketed term more rigorously. As outlined in Appendix C, the Taylor expansions for these equations provide a set of conditions for each order of $\epsilon$. According to Eq. (C14), the following relations are obtained at the current order:

$$
\begin{aligned}
& 0=\left.\frac{\partial \phi_{\alpha}^{0}}{\partial \eta}\right|_{\eta_{+}^{0}} \eta_{+}^{1}+\phi_{\alpha}^{1}\left(\eta_{+}^{0}\right), \\
& 0=\left.\frac{\partial \phi_{\alpha}^{0}}{\partial \eta}\right|_{\eta_{-}^{0}} \eta_{-}^{1}+\phi_{\alpha}^{1}\left(\eta_{-}^{0}\right) .
\end{aligned}
$$

Inserting $\partial \phi_{\alpha}^{0} /\left.\partial \eta\right|_{\eta_{ \pm}^{0}}=0$ yields the required condition, which validates the following statement [Eq. (74)]:

$$
\psi_{\alpha}\left(\mu^{0}\right)-\psi_{\beta}\left(\mu^{0}\right)=-\frac{v_{n}^{0}}{m_{\alpha \beta}}-\gamma_{\alpha \beta} \kappa_{0},
$$

which is the sought relation between curvature and the grandpotential driving force. Note that in the absence of the latter, which corresponds to two chemically identical phases (known as grains), a curvature-driven boundary migration of the form $v_{n}^{0}=-m_{\alpha \beta} \gamma_{\alpha \beta} \kappa_{0}$ is recovered by the effect of the Allen-Cahn (phase-field) equation. However, the general case may give the impression that the sharp-interface Gibbs-Thomson effect cannot be recovered since Eq. (77) differs significantly from Eq. (27). Moreover, there is a fundamental difference in the philosophies of the current model and the sharp interface theory that existed right from the beginning. This is that while 
Sec. III dealt with pure substances, the developed phase-field model pertains to alloys. Indeed, the current formulation is not directly a model for surface self-diffusion in a unicomponent solid-vapor system. That said, it is defended that it is its counterpart for multicomponent alloys with a striking level of exactness. Second, with a slight reinterpretation of various terms, it readily handles the pure substance case as well. The first of the above can be realized by considering the equilibrium case $v_{n}=0$. Then differentiating the appropriately reduced Eq. (77) gives

$$
\frac{\partial \kappa_{0}}{\partial \mu^{0}}=\gamma_{\alpha \beta}^{-1}\left[\frac{\partial \psi_{\beta}\left(\mu^{0}\right)}{\partial \mu^{0}}-\frac{\partial \psi_{\alpha}\left(\mu^{0}\right)}{\partial \mu^{0}}\right] .
$$

Since $\partial \psi_{\alpha}\left(\mu^{0}\right) / \partial \mu^{0}=-c^{\alpha} \forall \alpha$, one obtains

$$
\frac{\partial \mu^{0}}{\partial \kappa_{0}}=\frac{\gamma_{\alpha \beta}}{c^{\alpha}\left(\mu^{0}\right)-c^{\beta}\left(\mu^{0}\right)} .
$$

This is precisely the result Eq. (A7) derived from a more general Gibbs-Thomson condition outlined in Appendix A.

The second point can be seen by highlighting small shifts in the chemical potential. Therefore, the chemical driving force $\psi_{\alpha}-\psi_{\beta}$ is expanded as a deviation from the equilibrium

$$
\begin{aligned}
\psi_{\alpha}(\mu)-\psi_{\beta}(\mu)= & \left(\mu-\mu_{\mathrm{eq}}\right)\left[c^{\beta}\left(\mu_{\mathrm{eq}}\right)-c^{\alpha}\left(\mu_{\mathrm{eq}}\right)\right] \\
& +O\left[\left(\mu-\mu_{\mathrm{eq}}\right)^{2}\right],
\end{aligned}
$$

which yields

$$
\mu^{0}-\mu_{\mathrm{eq}}=\frac{\gamma_{\alpha \beta} \kappa_{0}+\frac{v_{n}^{0}}{m_{\alpha \beta}}}{c^{\alpha}\left(\mu_{\mathrm{eq}}\right)-c^{\beta}\left(\mu_{\mathrm{eq}}\right)}+O\left[\left(\mu^{0}-\mu_{\mathrm{eq}}\right)^{2}\right] .
$$

This is the much popular generalized Gibbs-Thomson relation for binary systems. Here please note the marked similarity between Eq. (81) and the chemical potential of the sharp interface, according to Eq. (36). If we treat $\left[c^{\alpha}\left(\mu_{\mathrm{eq}}\right)-c^{\beta}\left(\mu_{\mathrm{eq}}\right)\right] \mu^{0}$ as the chemical potential in Eq. (36), equivalent relations are obtained in the sense that $\gamma_{\alpha \beta}$ represents the physical interfacial energy $\gamma_{s}$ and $m_{\alpha \beta}$ denotes the physical mobility of the interface $m_{s}$. Therefore, the kinetic term $v_{n}^{0} / m_{\alpha \beta}$ reflects the limited attachment kinetics of the interface, imprinted through the phase-field evolution, which becomes evident from the sharp interface law [21]. The additional composition difference in the denominator results from the treatment of a binary incompressible system, instead of a unary compressible one, as discussed in Appendix A. Further details are discussed in Sec. VII C.

To study the nature of $\eta_{ \pm}^{1}$, the derivative boundary conditions [Eq. (42)] are utilized. By analogously expanding the powers of $\epsilon$ to Eq. (C14), at the first order, they read as

$$
\begin{aligned}
& 0=\left.\frac{\partial^{2} \phi_{\alpha}^{0}}{\partial \eta^{2}}\right|_{\eta_{+}^{0}} \eta_{+}^{1}+\left.\frac{\partial \phi_{\alpha}^{1}}{\partial \eta}\right|_{\eta_{+}^{0}}, \\
& 0=\left.\frac{\partial^{2} \phi_{\alpha}^{0}}{\partial \eta^{2}}\right|_{\eta_{-}^{0}} \eta_{-}^{1}+\left.\frac{\partial \phi_{\alpha}^{1}}{\partial \eta}\right|_{\eta_{-}^{0}},
\end{aligned}
$$

which results in

$$
\eta_{ \pm}^{1}=-\frac{\left.\frac{\partial \phi_{\alpha}^{1}}{\partial \eta}\right|_{\eta_{ \pm}^{0}}}{\left.\frac{\partial^{2} \phi_{\alpha}^{0}}{\partial \eta^{2}}\right|_{\eta_{ \pm}^{0}}}
$$

Once again, for consistency, it is important to show that $\left.\frac{\partial \phi_{\alpha}^{1}}{\partial \eta}\right|_{\eta_{ \pm}^{0}}$ is bounded, which is done in Appendix D.

\section{Chemical potential}

The chemical-potential equation at the order $\epsilon^{-2}$ reads as

$$
\frac{\partial}{\partial \eta}\left(M^{0} \frac{\partial \mu^{1}}{\partial \eta}\right)+\frac{\partial}{\partial \eta}\left(M^{1} \frac{\partial \mu^{0}}{\partial \eta}\right)+M^{0} \kappa_{0} \frac{\partial \mu^{0}}{\partial \eta}=0 .
$$

Since $\frac{\partial \mu^{0}}{\partial \eta}=0$, only a single term remains. Furthermore, $J^{1}(\eta)=M^{0} \partial \mu^{1} / \partial \eta$. Thus, we may integrate along the normal direction, to obtain

$$
J^{1}(\eta)=M\left(\phi_{\alpha}^{0}\right) \frac{\partial \mu^{1}}{\partial \eta}=C .
$$

Again, boundary conditions are required to obtain an appropriate value for $C$. The flux boundary condition next to the lowest order [Eq. (C28)] reads as

$$
\left.\frac{\partial J^{0}}{\partial \eta}\right|_{\eta_{ \pm}^{0}} \eta_{ \pm}^{1}+J^{1}\left(\eta_{ \pm}^{0}\right)=0
$$

Since $J^{0}(\eta)=0$, it further simplifies to $J^{1}\left(\eta_{ \pm}^{0}\right)=$ $\left[M^{0} \partial \mu^{1} / \partial \eta\right]_{\eta^{0}}=0$. Accordingly, $J^{1}(\eta)=C=0$, and $\mu^{1}$ is independent of $\eta$, which implies that $J^{2}(\eta)=M^{0} \partial \mu^{2} / \partial \eta$.

\section{F. Next order}

\section{Chemical potential}

At the order $1 / \epsilon$, Eq. (65) reads as

$$
\begin{aligned}
0= & \frac{\partial}{\partial \eta}\left(M^{0} \frac{\partial \mu^{2}}{\partial \eta}+M^{1} \frac{\partial \mu^{1}}{\partial \eta}+M^{2} \frac{\partial \mu^{0}}{\partial \eta}\right) \\
& +M^{0} \kappa_{0} \frac{\partial \mu^{1}}{\partial \eta}+M^{0} \frac{\partial^{2} \mu^{0}}{\partial s^{2}}+\frac{\partial M^{0}}{\partial s} \frac{\partial \mu^{0}}{\partial s} \\
& +\left(M^{1} \kappa_{0}-M^{0} \eta \kappa_{0}^{2}+\left.v_{n}^{0} \frac{\partial c}{\partial \mu}\right|_{\phi_{\alpha}^{0}, \mu^{0}}\right) \frac{\partial \mu^{0}}{\partial \eta} \\
& +\left(c^{\alpha}\left(\mu^{0}\right)-c^{\beta}\left(\mu^{0}\right)\right) v_{n}^{0} \frac{\partial h_{\alpha}\left(\phi_{\alpha}^{0}\right)}{\partial \eta} .
\end{aligned}
$$

Inserting $\frac{\partial \mu^{0}}{\partial \eta}=0, \frac{\partial \mu^{1}}{\partial \eta}=0$ and $\frac{\partial M^{0}}{\partial s}=0$, we obtain

$$
\begin{aligned}
0= & \frac{\partial}{\partial \eta}\left(M^{0} \frac{\partial \mu^{2}}{\partial \eta}\right)+M^{0} \frac{\partial^{2} \mu^{0}}{\partial s^{2}} \\
& +\left(c^{\alpha}\left(\mu^{0}\right)-c^{\beta}\left(\mu^{0}\right)\right) v_{n}^{0} \frac{\partial h_{\alpha}\left(\phi_{\alpha}^{0}\right)}{\partial \eta} .
\end{aligned}
$$

Integrating across the interface yields

$$
\begin{aligned}
0= & {\left[M^{0} \frac{\partial \mu^{2}}{\partial \eta}\right]_{\eta_{-}^{0}}^{\eta_{+}^{0}}+\bar{M}^{\alpha \beta} \int_{\eta_{-}^{0}}^{\eta_{+}^{0}} g\left(\phi_{\alpha}^{0}\right) d \eta \frac{\partial^{2} \mu^{0}}{\partial s^{2}} } \\
& +\left(c^{\alpha}\left(\mu^{0}\right)-c^{\beta}\left(\mu^{0}\right)\right) v_{n}^{0} \underbrace{\int_{\eta_{-}^{0}}^{\eta_{+}^{0}} \frac{\partial h_{\alpha}\left(\phi_{\alpha}^{0}\right)}{\partial \eta} d \eta}_{-1},
\end{aligned}
$$


TABLE I. Values for the integral $I_{g} \equiv \int_{\eta^{0}}^{\eta_{+}^{0}} g\left(\phi_{\alpha}^{0}\right) d \eta$, depending on the particular choice of $g$. $\Gamma$ denotes the gamma function.

\begin{tabular}{lc}
\hline \hline Mobility function $g\left(\phi_{\alpha}\right)$ & Integral $I_{g}$ \\
\hline$\sqrt{\phi_{\alpha}\left(1-\phi_{\alpha}\right)}$ & $\pi / 4$ \\
$\phi_{\alpha}\left(1-\phi_{\alpha}\right)$ & $\pi^{2} / 32$ \\
$\phi_{\alpha}^{2}\left(1-\phi_{\alpha}\right)^{2}$ & $3 \pi^{2} / 512$ \\
$\phi_{\alpha}^{n}\left(1-\phi_{\alpha}\right)^{n}$ & $\pi^{3 / 2} \Gamma(n+1 / 2) /\left[4^{n+1} \Gamma(n+1)\right]$ \\
\hline \hline
\end{tabular}

where the first bracketed term in the equation above is obtained from the boundary conditions [Eq. (C29)]

$$
\begin{aligned}
0= & \left.\frac{\partial^{2} J^{0}}{\partial \eta^{2}}\right|_{\eta_{ \pm}^{0}}\left(\eta_{ \pm}^{1}\right)^{2}+\left.2 \frac{\partial J^{0}}{\partial \eta}\right|_{\eta_{ \pm}^{0}} \eta_{ \pm}^{2} \\
& +\left.2 \frac{\partial J^{1}}{\partial \eta}\right|_{\eta_{ \pm}^{0}} \eta_{ \pm}^{1}+2 J^{2}\left(\eta_{ \pm}^{0}\right)+2 v_{n}^{0}\left[c\left(\eta_{ \pm}^{0}, \epsilon=0\right)-c^{ \pm}\right] .
\end{aligned}
$$

Substituting $J^{0}(\eta)=0$ and $J^{1}(\eta)=0$ results in

$$
J^{2}\left(\eta_{ \pm}^{0}\right)=\left[M^{0} \frac{\partial \mu^{2}}{\partial \eta}\right]_{\eta_{ \pm}^{0}}=v_{n}^{0}\left[c^{ \pm}-c\left(\eta_{ \pm}^{0}, \epsilon=0\right)\right] .
$$

Since $\phi_{\alpha}^{0}\left(\eta_{-}^{0}\right)=1$ and $\phi_{\alpha}^{0}\left(\eta_{+}^{0}\right)=0$, the compositions approached from the interface reduce to $c\left(\eta_{+}^{0}, \epsilon=0\right)=c^{\beta}\left(\mu^{0}\right)$ and $c\left(\eta_{-}^{0}, \epsilon=0\right)=c^{\alpha}\left(\mu^{0}\right)$, which leads to

$$
\begin{aligned}
& {\left[M^{0} \frac{\partial \mu^{2}}{\partial \eta}\right]_{\eta_{+}^{0}}=v_{n}^{0}\left[c^{\beta}\left(\mu^{+}\right)-c^{\beta}\left(\mu^{0}\right)\right],} \\
& {\left[M^{0} \frac{\partial \mu^{2}}{\partial \eta}\right]_{\eta_{-}^{0}}=v_{n}^{0}\left[c^{\alpha}\left(\mu^{-}\right)-c^{\alpha}\left(\mu^{0}\right)\right],}
\end{aligned}
$$

where the bulk $c^{ \pm}$have been written in terms of the bulk chemical potential.

The governing equation in the sharp interface limit therefore is found to be

$$
v_{n}^{0}=\frac{\bar{M}^{\alpha \beta} \int_{\eta_{-}^{0}}^{\eta_{+}^{0}} g\left(\phi_{\alpha}^{0}\right) d \eta}{c^{\alpha}\left(\mu^{-}\right)-c^{\beta}\left(\mu^{+}\right)} \frac{\partial^{2} \mu^{0}}{\partial s^{2}} .
$$

Interestingly, this equation is consistent with the balance law for an evolving surface [see Appendix B, Eq. (B4)]. Thereby the surface excess flux is identified to be $\boldsymbol{j}_{s}=$ $-\boldsymbol{s} \bar{M}^{\alpha \beta} \int_{\eta_{-}^{0}}^{\eta_{+}^{0}} g\left(\phi_{\alpha}^{0}\right) d \eta \partial \mu^{0} / \partial s$.

We introduce the quantity $I_{g} \equiv \int_{\eta_{-}^{0}}^{\eta_{+}^{0}} g\left(\phi_{\alpha}^{0}\right) d \eta$, which is just a nondimensional prefactor. For functions of the form $g\left(\phi_{\alpha}\right)=$ $\phi_{\alpha}^{n}\left(1-\phi_{\alpha}\right)^{n}$, the resulting values of $I_{g}$ are tabulated in Table I.

Assuming that the bulk compositions do not differ strongly from the equilibrium compositions, $c^{\alpha}\left(\mu^{-}\right)-c^{\beta}\left(\mu^{+}\right) \approx$ $c^{\alpha}\left(\mu_{\mathrm{eq}}\right)-c^{\beta}\left(\mu_{\mathrm{eq}}\right)$ yields

$$
v_{n}^{0} \approx \frac{\bar{M}^{\alpha \beta} I_{g}}{c^{\alpha}\left(\mu_{\mathrm{eq}}\right)-c^{\beta}\left(\mu_{\mathrm{eq}}\right)} \frac{\partial^{2} \mu^{0}}{\partial s^{2}} .
$$

Substituting Eq. (81) and rewriting it with the handy definition $\bar{\mu} \equiv\left[c^{\alpha}\left(\mu_{\mathrm{eq}}\right)-c^{\beta}\left(\mu_{\mathrm{eq}}\right)\right] \mu^{0}[\bar{\mu}$, which has the same unit as $\mu$ $\left.\left(\mathrm{J} / \mathrm{m}^{3}\right)\right]$, results in the following set of equations:

$$
\begin{gathered}
v_{n}^{0} \approx \frac{\bar{M}^{\alpha \beta} I_{g}}{\left[c^{\alpha}\left(\mu_{\mathrm{eq}}\right)-c^{\beta}\left(\mu_{\mathrm{eq}}\right)\right]^{2}} \frac{\partial^{2} \bar{\mu}}{\partial s^{2}}, \\
\bar{\mu} \approx \bar{\mu}_{\mathrm{eq}}+\gamma_{\alpha \beta} \kappa_{0}+\frac{v_{n}^{0}}{m_{\alpha \beta}},
\end{gathered}
$$

valid for small deviations from the equilibrium. Since the above equations take an equivalent form as the counterpart of the sharp interface [Eqs. (35) and (36)], coupled motion by surface diffusion and attachment kinetics is retrieved.

Furthermore, the rate constant for growth, dominated by surface diffusion, is obtained as

$$
B=\frac{\bar{M}^{\alpha \beta} I_{g} \gamma_{\alpha \beta}}{\left[c^{\alpha}\left(\mu_{\mathrm{eq}}\right)-c^{\beta}\left(\mu_{\mathrm{eq}}\right)\right]^{2}},
$$

which is the sought coefficient to relate the physical and model-specific timescale. According to Eq. (34), the characteristic length scale for the attachment kinetics is expressed as $l_{c}=\sqrt{B /\left(\gamma_{\alpha \beta} m_{\alpha \beta}\right)}$, in terms of the model parameters.

Often, it is desired to handle the case of infinite rather than finite attachment kinetics. This can be achieved through a slight modification of the model. Here the mobility $m_{\alpha \beta}$ is no longer treated as independent of $\epsilon$ but is replaced by $m_{\alpha \beta}=\tilde{m}_{\alpha \beta} / \epsilon$, where $\tilde{m}_{\alpha \beta}$ is a constant. Accordingly, one obtains infinite attachment kinetics in the sharp-interface limit since $\lim _{\epsilon \rightarrow 0} l_{c}$ becomes zero. More technically speaking, the first term inside the parentheses, in Eq. (70), which appears instead at one higher order, can be dropped.

\section{G. Higher-order contributions to the phase-field profile}

According to the preliminary analysis, the choice of the interpolation function $h_{\alpha}$ does not alter the simulation result, provided it meets the imposed criteria. Therefore, the user has freedom to choose any suitable interpolation function that provides ease of implementation or low computational costs. Accordingly, the rather naive choice $h_{\alpha}=\phi_{\alpha}$ may be deemed appropriate. To test the validity of the derived equations, a simulation of a small cylindrical particle (radius $r=2 \epsilon$ ) is performed until an equilibrium state is reached, as shown in Fig. 1. The observed radial phase-field profile and the profile of $\phi_{\alpha}^{0}$ [Eq. (68)] are shown in Fig. 2. Note that even in such an artificially, strongly perturbed state, which is due to the large interfacial width $\epsilon$, a fairly small deviation of the simulation equilibrium profile from the profile of $\phi_{\alpha}^{0}$ is observed. Despite this naive approach, this demonstrates the stability of the interface and reflects the absence of unwanted excess energy in the current model. However, in zoomed regions of the profile, in the vicinity of the bulk, small deviations are observed. The corresponding reason may be identified from Eq. (70), where the differential equation for $\phi_{\alpha}^{1}(\eta)$ depends on the particular nature of $h_{\alpha}$. According to the ansatz [Eq. (55)], and given a finite interfacial width, the contribution of the interpolation function to the phase-field profile becomes increasingly important. Since a finite interface width and a rather small particle are analyzed, the inconsistencies may be related to the particular form of the higher-order solutions $\phi_{\alpha}^{1}(\eta), \phi_{\alpha}^{2}(\eta)$. 


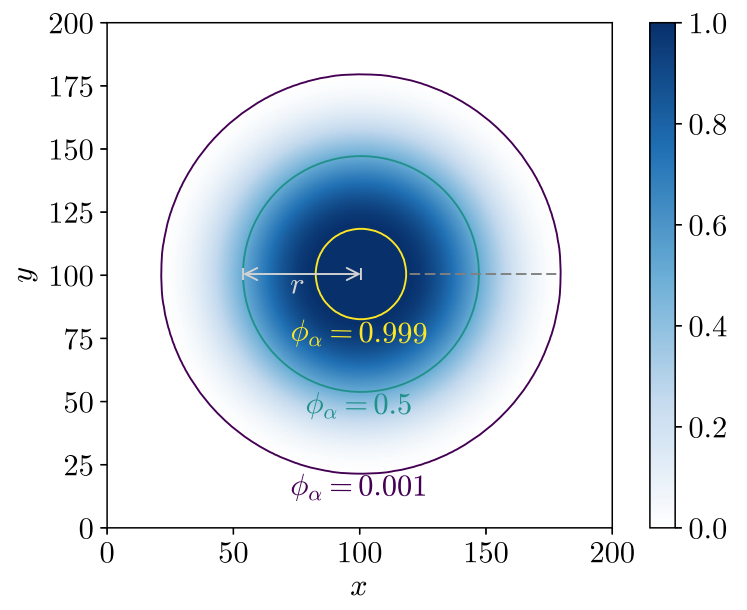

FIG. 1. Equilibrium phase field $\phi_{\alpha}$, for a cylindrical particle of radius $r$, with a relatively large interfacial width $(\epsilon / r=1 / 2)$ in the current model. The solid lines indicate the isolines of the phase field, and the dashed line represents a radial cross section of the interface.

Therefore, further analysis is performed in the following, to identify higher-order corrections in the phase-field profile.

Since each interpolation function has to be treated individually from now on, presenting the solution at higher orders in a generic form becomes a tedious task. Therefore, this section exemplarily focuses on the particular choice $h_{\alpha}=\phi_{\alpha}$. The differential equation for $\phi_{\alpha}^{1}(\eta)$ can now be written as

$$
\frac{\partial^{2} \phi_{\alpha}^{1}}{\partial \eta^{2}}+\frac{16}{\pi^{2}} \phi_{\alpha}^{1}=\left(\frac{-v_{n}^{0}}{m_{\alpha \beta} \gamma_{\alpha \beta}}-\kappa_{0}\right)\left(\frac{\partial \phi_{\alpha}^{0}}{\partial \eta}+\frac{1}{2}\right) .
$$

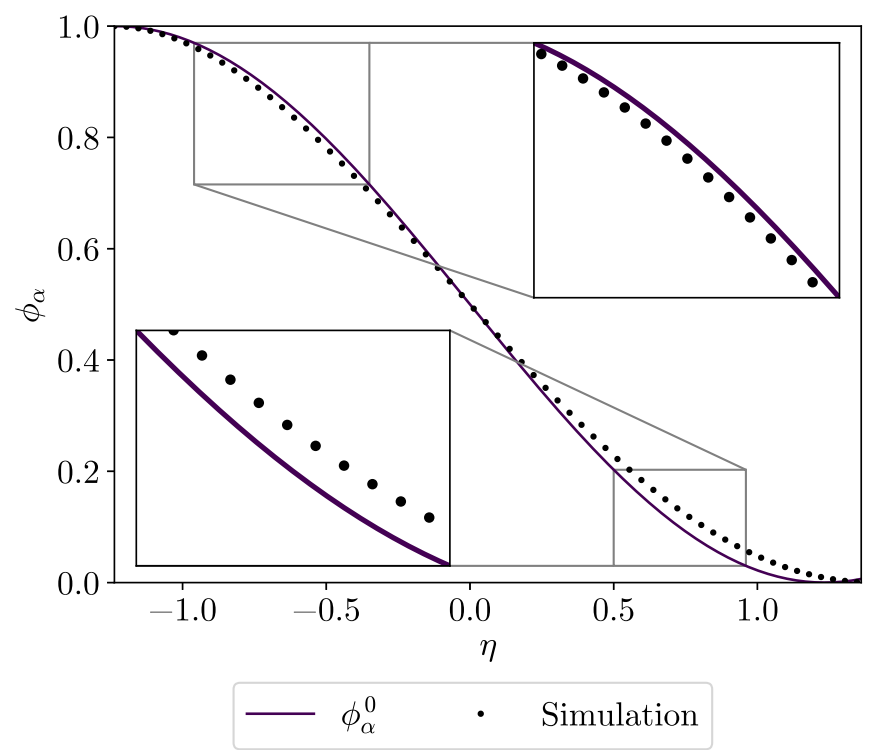

FIG. 2. Simulated equilibrium phase-field profile $\phi_{\alpha}$ along the radial direction (dashed line in Fig. 1), rescaled in terms of $\eta$ (solid symbols). The profile of $\phi_{\alpha}^{0}$ is shown as a solid line.
By defining $a \equiv-v_{n}^{0} /\left(m_{\alpha \beta} \gamma_{\alpha \beta}\right)-\kappa_{0}$, the general solution to this differential equation reads as

$$
\phi_{\alpha}^{1}=a\left[\frac{\pi^{2}}{32}+\left(K_{1}-\frac{\eta}{4}\right) \sin \left(\frac{4}{\pi} \eta\right)+K_{2} \cos \left(\frac{4}{\pi} \eta\right)\right],
$$

where $K_{1}$ and $K_{2}$ are two undetermined constants. From the condition $\phi_{\alpha}(\eta=0)=1 / 2$, it follows that $K_{2}=-\pi^{2} / 32$. Equations (75) and (76) provide the boundary conditions $\phi_{\alpha}^{1}\left(\eta_{ \pm}^{0}\right)=0$ at either end, to obtain $K_{1}=0$. The first-order correction of the phase-field profile then writes as

$$
\phi_{\alpha}^{1}=a\left[\frac{\pi^{2}}{32}-\frac{\eta}{4} \sin \left(\frac{4}{\pi} \eta\right)-\frac{\pi^{2}}{32} \cos \left(\frac{4}{\pi} \eta\right)\right] .
$$

At the first order $\eta_{ \pm}^{1}$, the shift of the endpoints is found by first taking the derivative

$$
\frac{\partial \phi_{\alpha}^{1}}{\partial \eta}=a\left[\left(\frac{\pi}{8}-\frac{1}{4}\right) \sin \left(\frac{4}{\pi} \eta\right)-\frac{\eta}{\pi} \cos \left(\frac{4}{\pi} \eta\right)\right]
$$

to obtain $\partial \phi_{\alpha}^{1} /\left.\partial \eta\right|_{\eta_{ \pm}^{0}}= \pm a(\pi / 8-1 / 4)$. Finally, substituting $\partial \phi_{\alpha}^{1} /\left.\partial \eta\right|_{\eta_{ \pm}^{0}}$ and $\partial^{2} \phi_{\alpha}^{0} /\left.\partial \eta^{2}\right|_{\eta_{ \pm}^{0}}= \pm 8 / \pi^{2}$ into Eq. (84) yields the finite values

$$
\eta_{+}^{1}=\eta_{-}^{1}=\frac{a \pi^{2}}{32}\left(1-\frac{\pi}{2}\right) .
$$

Therefore, the first-order correction of the phase field has been thoroughly presented. To conserve the boundary conditions at the order $\epsilon^{2}$, we obtain [see Eq. (C15)]

$$
0=\left.\frac{\partial^{2} \phi_{\alpha}^{0}}{\partial \eta^{2}}\right|_{\eta_{ \pm}^{0}}\left(\eta_{ \pm}^{1}\right)^{2}+\left.2 \frac{\partial \phi_{\alpha}^{1}}{\partial \eta}\right|_{\eta_{ \pm}^{0}} \eta_{ \pm}^{1}+2 \phi_{\alpha}^{2}\left(\eta_{ \pm}^{0}\right)
$$

which, by substituting Eq. (84), simplifies to

$$
\phi_{\alpha}^{2}\left(\eta_{ \pm}^{0}\right)=-\left.\frac{1}{2} \frac{\partial \phi_{\alpha}^{1}}{\partial \eta}\right|_{\eta_{ \pm}^{0}} \eta_{ \pm}^{1} .
$$

Interestingly, the second-order phase-field correction does not vanish at the zeroth-order endpoints, but takes the finite values

$$
\phi_{\alpha}^{2}\left(\eta_{ \pm}^{0}\right)= \pm \phi_{\alpha}^{2}\left(\eta_{+}^{0}\right)= \pm \frac{a^{2} \pi^{2}}{256}\left(\frac{\pi}{2}-1\right)^{2} .
$$

To identify the contribution of the second-order correction, the phase-field equation at the order $\epsilon$, which reads as

$$
\begin{aligned}
\frac{\partial^{2} \phi_{\alpha}^{2}}{\partial \eta^{2}}+\frac{16}{\pi^{2}} \phi_{\alpha}^{2}= & a \frac{\partial \phi_{\alpha}^{1}}{\partial \eta}-\frac{v_{n}^{1}}{m_{\alpha \beta} \gamma_{\alpha \beta}} \frac{\partial \phi_{\alpha}^{0}}{\partial \eta}+\frac{\stackrel{\circ}{\alpha}_{\alpha}^{0}}{m_{\alpha \beta} \gamma_{\alpha \beta}}-\frac{\partial^{2} \phi_{\alpha}^{0}}{\partial s^{2}} \\
& +\eta \kappa_{0}^{2} \frac{\partial \phi_{\alpha}^{0}}{\partial \eta}+\mu^{1} \frac{c^{\beta}\left(\mu_{\mathrm{eq}}\right)-c^{\alpha}\left(\mu_{\mathrm{eq}}\right)}{2 \gamma_{\alpha \beta}}
\end{aligned}
$$

is analyzed, where the last term appears in an expanded form, according to Eq. (80). Since $\AA^{0}=0$ and $\partial^{2} \phi_{\alpha}^{0} / \partial s^{2}=0$, both terms can be neglected subsequently. Applying the same steps again, i.e., multiplying by $\partial \phi_{\alpha}^{0} / \partial \eta$ and integrating, yields

$$
\mu^{1}=\frac{v_{n}^{1}}{m_{\alpha \beta}\left[c^{\alpha}\left(\mu_{\mathrm{eq}}\right)-c^{\beta}\left(\mu_{\mathrm{eq}}\right)\right]} .
$$


Defining $b \equiv v_{n}^{1} /\left(m_{\alpha \beta} \gamma_{\alpha \beta}\right)$, the solution of Eq. (108), which is subject to the boundary conditions (107), reads as

$$
\begin{gathered}
\phi_{\alpha}^{2}=p_{s}(\eta) \sin \left(\frac{4}{\pi} \eta\right)+p_{c}(\eta) \cos \left(\frac{4}{\pi} \eta\right)-\frac{\pi^{2} b}{32}, \\
p_{s}(\eta) \equiv \frac{1}{512}\left[a^{2}\left(2 \pi^{2}-2 \pi^{3}+\pi^{4}-32 \eta^{2}\right)\right. \\
\left.+128 b \eta+\kappa_{0}^{2}\left(\pi^{4}-64 \eta^{2}\right)\right], \\
p_{c}(\eta) \equiv \frac{\pi}{64}\left[2 \pi b+\left((1-\pi) a^{2}-2 \kappa_{0}^{2}\right) \eta\right] .
\end{gathered}
$$

For a stationary profile $\left(v_{n}=0, a^{2}=\kappa_{0}^{2}, b=0\right)$, it simplifies to

$$
\begin{aligned}
\phi_{\alpha}^{2}= & \frac{\kappa_{0}^{2}}{256}\left[\left(\pi^{2}-\pi^{3}+\pi^{4}-48 \eta^{2}\right) \sin \left(\frac{4}{\pi} \eta\right)\right. \\
& \left.-4 \pi(1+\pi) \eta \cos \left(\frac{4}{\pi} \eta\right)\right] .
\end{aligned}
$$

The shift of the interfacial endpoints $\eta_{ \pm}$, induced by the second-order correction, is expressed as follows, by the use of the boundary condition (42), at the proper order (the derivation is outlined in Appendix C):

$$
\eta_{ \pm}^{2}=-\frac{\left.\frac{1}{2} \frac{\partial^{3} \phi_{\alpha}^{0}}{\partial \eta^{3}}\right|_{\eta_{ \pm}^{0}}\left(\eta_{ \pm}^{1}\right)^{2}+\left.\frac{\partial^{2} \phi_{\alpha}^{1}}{\partial \eta^{2}}\right|_{\eta_{ \pm}^{0}} \eta_{ \pm}^{1}+\left.\frac{\partial \phi_{\alpha}^{2}}{\partial \eta}\right|_{\eta_{ \pm}^{0}}}{\left.\frac{\partial^{2} \phi_{\alpha}^{0}}{\partial \eta^{2}}\right|_{\eta_{ \pm}^{0}}}
$$

which, for the stationary case, results in

$$
\eta_{ \pm}^{2}= \pm \kappa_{0}^{2} \frac{3 \pi^{4}}{1024}
$$

Including this term in the expanded form, we obtain up to order $O\left(\epsilon^{2}\right)$ :

$$
\begin{gathered}
\eta_{+}=\frac{\pi^{2}}{8}+\epsilon \kappa_{0} \frac{\pi^{2}}{32}\left(\frac{\pi}{2}-1\right)+\epsilon^{2} \kappa_{0}^{2} \frac{3 \pi^{4}}{1024}, \\
\eta_{-}=-\frac{\pi^{2}}{8}+\epsilon \kappa_{0} \frac{\pi^{2}}{32}\left(\frac{\pi}{2}-1\right)-\epsilon^{2} \kappa_{0}^{2} \frac{3 \pi^{4}}{1024} .
\end{gathered}
$$

It is interesting to note that the amount of perturbation in the stationary state is just a function of $\epsilon \kappa_{0}$, i.e., the ratio of the interfacial width to the radius of curvature. This reflects the common understanding that the length scale of the problem must be much larger than the interfacial thickness, in order to ensure the validity of the sharp interface limit.

In Fig. 3 the derived solutions for $\phi_{\alpha}$ are shown up to the second order, in addition to the simulated profile. Now the deviations from the profile of $\phi_{\alpha}^{0}$ are very well explained by taking the higher-order corrections $\phi_{\alpha}^{1}$ and $\phi_{\alpha}^{2}$ (dashed and solid lines, respectively) into account. In contrast to the right end $(\eta>0)$, where the observed profile clearly overshoots the sharp-interface profile, the interfacial endpoint of the simulated profile at the left end $(\eta<0)$, where $\phi_{\alpha}$ approaches unity, matches much better with the zeroth-order profile. By inspecting Eqs. (116) and (117) correspondingly, the following values are obtained for the shown setup $\left(\epsilon \kappa_{0}=0.5\right)$ :

$$
\begin{aligned}
& \eta_{+}-\eta_{+}^{0} \approx 0.1593, \\
& \eta_{-}-\eta_{-}^{0} \approx 0.0167 .
\end{aligned}
$$

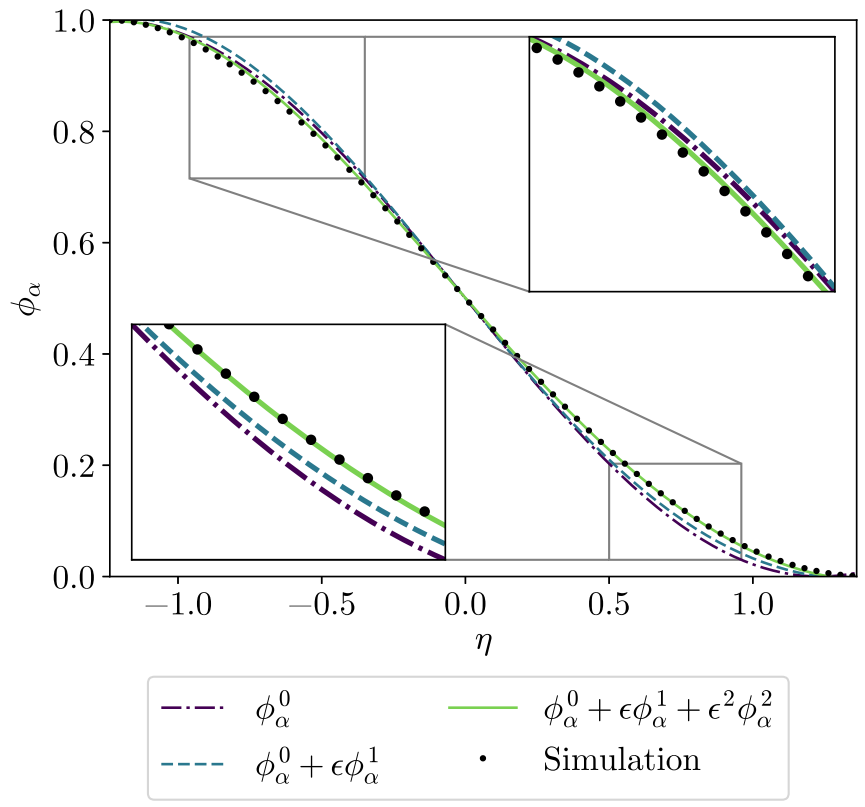

FIG. 3. Simulated equilibrium phase-field profile $\phi_{\alpha}$, along the radial direction (dashed line in Fig. 1), rescaled in terms of $\eta$ (points). The profiles derived as in the asymptotic analysis are additionally shown as lines. The simulation result almost perfectly matches the derived profile, including the second-order corrections in $\epsilon$. The value of $\kappa_{0}=1 / r$ is calculated from the isoline $\phi_{\alpha}=0.5$ of the simulation data (see Fig. 1).

Since the deviation for the left side $\left(\eta_{-}-\eta_{-}^{0}\right)$ is much smaller than the deviation for the right side, the theoretical treatment successfully predicts the observed behavior.

In this way the simulation result supports the validity of the derived equations, which bases on the correct incorporation of boundary conditions (Appendix C).

\section{THERMAL GROOVING}

\section{A. Sharp-interface treatment}

Pioneering work in the field of thermal grooving goes back to Mullins [19,105], where the formation of a groove at the grain-boundary intersection with a free surface was studied theoretically, under a variety of possible masstransfer mechanisms (surface diffusion, volume diffusion, evaporation-condensation). Following the analysis of Mullins, thermal grooving has been studied experimentally for copper [106,107], NiAl [108], tungsten [109-114], and alumina and magnesia [115], as well as many others. Experimentally, the study of thermal grooves is appealing, due to several reasons. It allows the ascertainment of diffusivities and the underlying diffusion mechanism, by analyzing the temporal behavior of the groove geometry, as well as the determination of interfacial energy ratios, by the measurement of triple-junction angles at the groove root. Furthermore, thermal grooves may interact with a moving grain boundary, as studied in [116-121], for example, which may lead to a drag effect, acting as a possible source to hinder grain growth. In order to find an analytical solution for grooving governed by surface diffusion, a so-called small-slope approximation was applied 
in the work of Mullins [19]. Accordingly, curvature depends only on the second derivative of the groove profile

$$
\kappa_{s} \approx-y^{\prime \prime},
$$

under the assumption that $\left|y^{\prime}\right|$ is negligibly small. Based on Eq. (31), a differential equation could be derived as

$$
\dot{y}=-B y^{\prime \prime \prime \prime}
$$

and could be solved, subject to the initial condition of a flat surface

$$
y(x, 0)=0,
$$

using the Laplace transform. Boundary conditions that read

$$
\begin{aligned}
& y^{\prime}(0, t)=m, \\
& y^{\prime \prime \prime}(0, t)=0,
\end{aligned}
$$

were applied, where $m$ is the slope of the groove at the triple junction. A grain boundary intersects the surface at the triple junction (per definition at $x=0$ ), which corresponds to the root of the groove. In terms of this slope, the equilibrium angle $\theta$ may be expressed as

$$
m=\tan \theta,
$$

while from the balance of the forces at the triple junction [97], the following relations hold:

$$
\begin{gathered}
\theta=\arcsin \frac{\gamma_{\mathrm{GB}}}{2 \gamma_{s}}, \\
m=\left[4\left(\frac{\gamma_{s}}{\gamma_{\mathrm{GB}}}\right)^{2}-1\right]^{-\frac{1}{2}} .
\end{gathered}
$$

Moreover, it was found that the temporally and spatially dependent solution can be written in a time-independent form, $z(u)$, using

$$
y(x, t)=m(B t)^{\frac{1}{4}} z(u) .
$$

The rescaled $x$ coordinate $u$ was introduced as

$$
u \equiv \frac{x}{(B t)^{\frac{1}{4}}},
$$

and thus the groove profile behaves self-similarly, while the size of the groove temporally follows a power law with the exponent $n=1 / 4$. Moreover, the particular groove shape obeys the following ordinary differential equation:

$$
z^{\prime \prime \prime \prime}-\frac{1}{4} u z^{\prime}+\frac{1}{4} z=0,
$$

where the spatial differentiation is performed with respect to $u$. The solution was stated as a power series

$$
z(u)=\sum_{n=0}^{\infty} a_{n} u^{n},
$$

with the coefficients chosen as tabulated in Table II. In Robertson's later extension of the work of Mullins, [20], the groove profile was solved numerically, without the necessity of any small-slope approximation. It was shown that the temporal development of the groove for finite slopes follows the same temporal law as stated in Eq. (128). The change in the equations, which is due to the finite surface slopes, is reflected
TABLE II. Polynomial coefficients for the time-independent solution $z(u)$,for surface diffusion, by Mullins [19]. $\Gamma$ represents the gamma function.

\begin{tabular}{lc}
\hline \hline$n$ & $a_{n}$ \\
\hline 0 & $\frac{-1}{\sqrt{2} \Gamma\left(\frac{5}{4}\right)}$ \\
1 & 1 \\
2 & $\frac{-1}{2 \sqrt{2} \Gamma\left(\frac{3}{4}\right)}$ \\
3 & 0 \\
$\geqslant 4$ & $a_{n-4} \frac{n-5}{4(n-3)(n-2)(n-1) n}$ \\
\hline \hline
\end{tabular}

only in a more complex form of the differential equation for $z(u)$, which rewrites to

$$
\begin{aligned}
& z^{\prime \prime \prime \prime}+\frac{1}{4}\left[\left(z-u z^{\prime}\right)\left(1+m^{2} z^{\prime 2}\right)^{2}\right] \\
& -\frac{m^{2} z^{\prime \prime}}{1+m^{2} z^{\prime 2}}\left[10 z^{\prime} z^{\prime \prime \prime}+\frac{3 z^{\prime \prime 2}\left(1-5 m^{2} z^{\prime 2}\right)}{1+m^{2} z^{\prime 2}}\right]=0 .
\end{aligned}
$$

In the limit $m \rightarrow 0$, it is easy to see that Eq. (132) becomes equivalent to Eq. (130). As a result, a unique solution, $z(u)$, is obtained for each particular value of $m$, due to the solution approach, which, however, cannot be presented in an exact analytical form, as given by the power series in Eq. (131). The numerical results were presented in a graphical form, which makes it difficult to make an exact comparison with the phasefield results. In order to obtain the correct groove shapes, $z(u)$, we repeated the process of numerically solving Eq. (132) for various values of $m$, subject to the boundary conditions

$$
\begin{gathered}
z^{\prime}(0)=1, \\
z^{\prime \prime \prime}(0)=3 m^{2} \frac{z^{\prime \prime 2}(0)}{1+m^{2}}, \\
z^{\prime \prime}(40)=0, \\
z(40)=0,
\end{gathered}
$$

which are equivalent to the boundary conditions stated in the original publication of Robertson [20]. Despite the sole difference that we use a cutoff at $u=40$, instead of $u=10$, to apply the boundary conditions at the right end of the groove (which in exact form would be applied at $u \rightarrow \infty$ ). We apply an iterative finite-difference scheme, for the discretization of all derivatives, using central differences up to the order of four, with a grid size of $\Delta u=0.02$. The Newton-Raphson method is applied by starting from an initial guess, $z^{0}$, before solving several subiterations until the solution converges and Eq. (132) is properly satisfied. At either end of the calculation domain, two boundary cells are updated automatically during each Newton-Raphson subiteration, to guarantee that all boundary conditions [Eqs. (133)-(136)] are satisfied.

The obtained groove geometries are depicted in a rescaled coordinate system (Fig. 4). Note that, as desired, the numerical solution for $\theta=0$ coincides with the analytical solution in the small-slope framework. Furthermore, a variety of groove shapes is obtained by employing the numerical scheme. However, despite the quantitative differences, the qualitative behavior of grooving does not become significantly different. Specifically in the vicinity of the triple junction, 


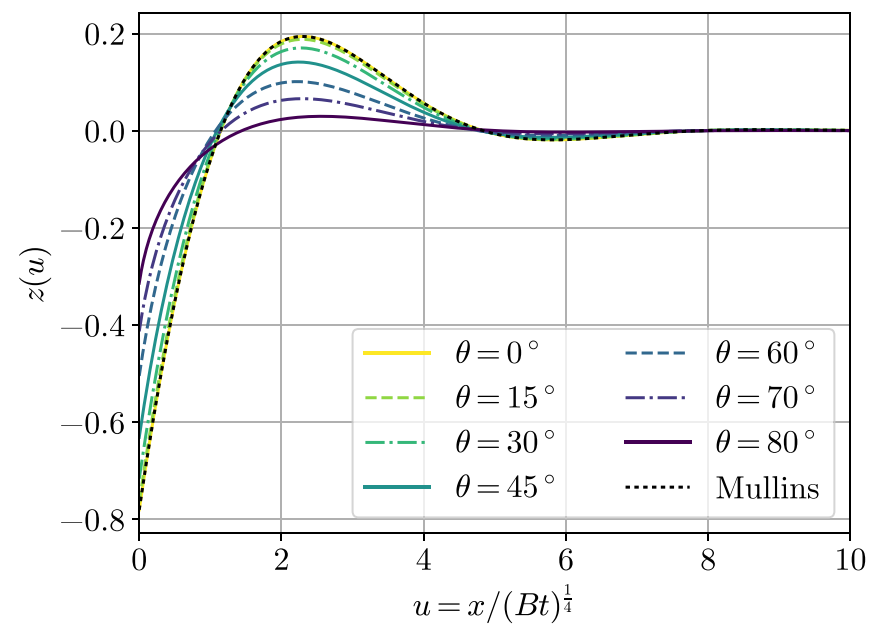

FIG. 4. Numerically solved groove profiles, $z(u)$, under surface diffusion. Seven profiles are shown for different values of $m=\tan \theta$, calculated in a finite difference scheme, according to [20]. An additional curve of the analytical solution by Mullins [19] is shown, which perfectly coincides with the numerical solution for $\theta=0^{\circ}$.

deepening is observed, while a hill forms and a global maximum is assumed, with an increasing distance from the root. In contrast to the volume diffusion-governed grooving in the small-slope framework [105], as shown in Fig. 5, the characteristic features of the grooves, caused by surface diffusion, are apparent. First, the temporal behavior during volume diffusion-governed growth is fundamentally different, since the size of the groove follows a different temporal power law with the exponent $1 / 3$. Therefore, the rescaling in Fig. 5 is applied with $t^{1 / 3}$, while $A^{\prime}$, similar to $B$, represents the physical rate constant of the bulk diffusion. Henceforth, a growth with an exponent of $1 / 4$ is deemed characteristic for thermal grooving by surface diffusion. Furthermore, in all surface diffusion grooves, following the hill, a characteristic dip is formed, corresponding to a local minimum in the groove shape. In volume diffusion grooves, such a dip is absent, whereas the groove slowly converges from above towards the original flat

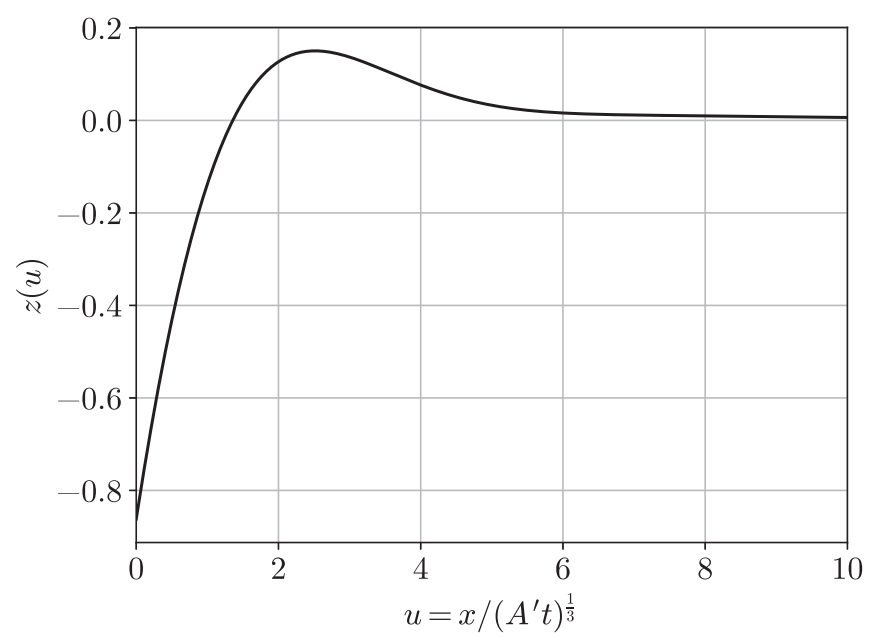

FIG. 5. Analytical groove profile for volume diffusion-governed grooving in the small-slope framework [105].

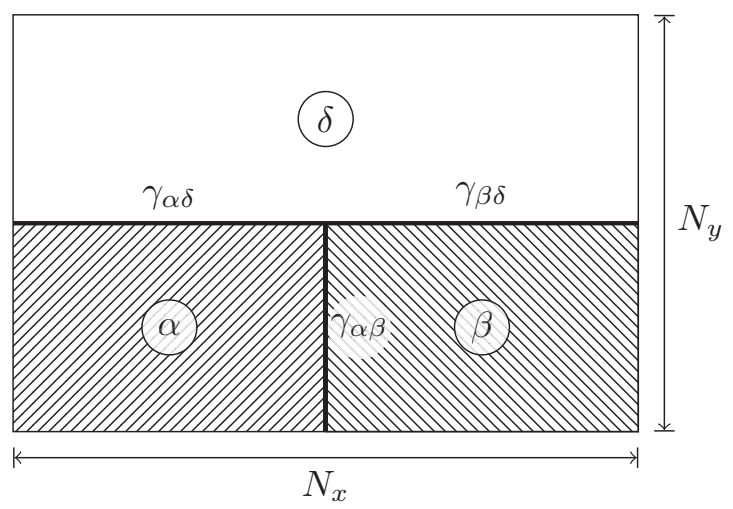

FIG. 6. Initial setup used for the simulations performed in this work.

position. This difference serves as an additional indicator to differentiate between surface and volume-diffusion governed grooving.

\section{B. Phase-field simulation}

In the current section, a system comprising three order parameters, $\phi_{\alpha}, \phi_{\beta}$, and $\phi_{\delta}(N=3)$, is considered, while a single composition field $(K=2)$ serves to incorporate diffusional transport into the system, where composition-related subscripts are omitted accordingly. A simulation setup as depicted in Fig. 6 is utilized. The two-dimensional simulation domain is symmetrically and vertically split into two respective regions of different composition, $c_{\mathrm{eq}}^{\alpha}=c_{\mathrm{eq}}^{\beta}=c_{\mathrm{eq}}^{S}$ and $c_{\mathrm{eq}}^{\delta}$. The area with the lower composition $c_{\text {eq }}^{\delta}$ belongs to a single vapor phase $\delta$, whereas the area with the higher composition represents a metallic solid that undergoes thermal grooving. The notion of a composition for a vapor-solid system is discussed in Appendix A 3. The solid contains two equally sized rectangular grains, $\alpha$ and $\beta$, which are separated by a common grain boundary with the interfacial energy $\gamma_{\alpha \beta}=\gamma_{\mathrm{GB}}$. The utilized parameter set is tabulated in Table III. To keep the results general, all quantities are expressed in an arbitrary unit system. Here $u_{E} \equiv \lambda_{E} \mathrm{~J}$ is an energy unit with a dimensionless prefactor $\lambda_{E}$, where J denotes Joule. Similarly, $u_{l} \equiv \lambda_{l} \mathrm{~m}$ and $u_{t} \equiv \lambda_{t}$ s can be written by dimensionless quantitites, $\lambda_{l}$ and $\lambda_{t}$, in terms of their SI counterparts. The nondimensional factors can be affixed by assigning the properties of a certain material to the values in Table III.

Equal interfacial energies $\left(\gamma_{\alpha \delta}=\gamma_{\beta \delta}=\gamma_{s}\right)$ and a common interfacial diffusivity prefactor, $\bar{M}^{\alpha \delta}=\bar{M}^{\beta \delta}$, are assigned to both horizontally aligned phase boundaries, respectively. In order to neglect bulk diffusion, the parameters $M^{\alpha}=M^{\beta}=$ $M^{\delta}$ were chosen to be zero. A simulation domain with a width of $N_{x}=2000$ cells and a height of $N_{y}=200$ cells has been chosen. Periodic boundary conditions are applied in the $x$ direction, so that two boundary grooves form at a distance of half the width of the domain. In the $y$ direction, zero Neumann (no-flux) boundary conditions are applied to fix the position of the grain boundary. The discretization spacing was chosen to be $\Delta x=\Delta y=0.25 u_{l}$. To minimize the effect of the attachment kinetics, the boundary mobilities are chosen as $m_{\alpha \beta}=m_{\alpha \delta}=m_{\beta \delta}=1 u_{l}^{4} /\left(u_{E} u_{t}\right)$. To verify that the attachment kinetics are subdominant, it should be noted that the 
TABLE III. Parameter set utilized for the simulations performed in the current work.

\begin{tabular}{lcc}
\hline \hline Parameter & Symbol & Value(s) \\
\hline Equil.-comp. left & $c_{\mathrm{eq}}^{\alpha \mathrm{a}}$ & 0.9 \\
Equil.-comp. right & $c_{\mathrm{eq}}^{\beta \mathrm{a}}$ & 0.9 \\
Equil.-comp. vapor & $c_{\mathrm{eq}}^{\delta}$ & 0.1 \\
Interpolation function & $h_{\alpha}(\boldsymbol{\phi})$ & $\phi_{\alpha}$ \\
Surface diff. mobility function & $g\left(\phi_{\alpha}, \phi_{\beta}\right)$ & $4 / \pi \sqrt{\phi_{\alpha} \phi_{\beta}}$ \\
Surface diff. prefactor left & $\bar{M}^{\alpha \delta}$ & $0.1 \frac{u_{l}^{6}}{u_{E} u_{t}}$ \\
Surface diff. prefactor right & $\bar{M}^{\beta \delta}$ & $0.1 \frac{u_{l}^{6}}{u_{E} u_{t}}$ \\
Grain boundary diff. prefactor & $\bar{M}^{\alpha \beta}$ & 0 \\
Bulk diffusion mobilities & $M^{\alpha, \beta, \delta}$ & 0 \\
Interface mobility (left) & $m_{\alpha \delta}^{\mathrm{b}}$ & $1 \frac{u_{l}^{4}}{u_{E} u_{t}}$ \\
Interface mobility (right) & $m_{\beta \delta}{ }^{\mathrm{b}}$ & $1 \frac{u_{l}^{4}}{u_{E} u_{t}}$ \\
Interface mobility (GB) & $m_{\alpha \beta}$ & $1 \frac{u_{l}^{4}}{u_{E} u_{t}}$ \\
Surface energy (left) & $\gamma_{\alpha \delta}^{\mathrm{c}}$ & $1 \frac{u_{E}}{u_{l}^{2}}$ \\
Surface energy (right) & $\gamma_{\beta \delta}^{\mathrm{c}}$ & $1 \frac{u_{E}}{u_{l}^{2}}$ \\
Grain boundary energy & $\gamma_{\alpha \beta}{ }^{\mathrm{d}}$ & $0.5 \ldots 1.875 \frac{u_{E}}{u_{l}^{2}}$ \\
Thermodynamic prefactor & $A$ & $50 \frac{u_{E}}{u_{l}^{3}}$ \\
Interfacial thickness parameter & $\epsilon$ & $2 u_{l}$ \\
Grid spacing & $\Delta x=\Delta y$ & $0.25 u_{l}$ \\
\hline \hline
\end{tabular}

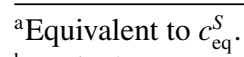

${ }^{\mathrm{b}}$ Equivalent to $m_{s}$.

${ }^{\mathrm{c} E q u i v a l e n t ~ t o ~} \gamma_{s}$.

${ }^{\mathrm{d}}$ Equivalent to $\gamma_{\mathrm{GB}}$.

length scale, at which the attachment kinetics operate, is much smaller than the interface width $\left(l_{c}<<\pi^{2} \epsilon / 4\right)$. This can be evaluated through Eq. (99) and $l_{c}=\sqrt{B /\left(\gamma_{s} m_{s}\right)}$. Inserting the values from Table III yields $l_{c} \approx 0.4 u_{l}<<\pi^{2} \epsilon / 4 \approx 5 u_{l}$.

\section{Volume conservation}

To model phase transformation, quantitative driving forces are introduced in various ways. Particularly, the incorporation of CALPHAD data is achieved either by parallel computing or by employing a linearization scheme [122-124]. What is different for these direct techniques is the fact that the quantitative data can be introduced through a numerical approximation [78,87,93]. Accordingly, the Gibbs free energy of a multicomponent phase $\alpha$ is expressed as

$$
\mathcal{G}^{\alpha}=\sum_{i<j}^{K-1, K-1} \mathcal{A}_{i j}^{\alpha} c_{i} c_{j}+\sum_{i}^{K-1} \mathcal{B}_{i}^{\alpha} c_{i}+\mathcal{C}^{\alpha},
$$

where the coefficients $\mathcal{A}_{i j}^{\alpha}, \mathcal{B}_{i}^{\alpha}$ and $\mathcal{C}^{\alpha}$ are determined appropriately to corroborate the CALPHAD data. Moreover, the accuracy of the coefficients is verified through the NewtonRaphson technique. Assuming that the molar volumes $\left(V_{m}\right)$ of the constituent phases are equal, the Gibbs free energy is transformed to Helmholtz free energy, through the relation $f^{\alpha}=\mathcal{G}^{\alpha} / V_{m}$.

By incorporating the CALPHAD data through Eq. (137), a chemical equilibrium can be established between the phases, by assigning equilibrium compositions. Since the grand potentials of the different phases are equal in chemical equilibrium, the driving force for any phase transformation becomes insignificant. By assigning equilibrium compositions to the phases, despite any morphological change, induced by the interface contribution, the volume fraction of the constituent phases can be preserved [94].

In order to treat the material equivalently to the sharp interface description in the following simulations, the free energies for each phase $\alpha \in\{\alpha, \beta, \delta\}$ are taken as

$$
f_{\alpha}(c)=A\left(c-c_{\mathrm{eq}}^{\alpha}\right)^{2},
$$

where $A$ is a parameter which controls the strength of the immiscibility of phases with different equilibrium compositions $c_{\text {eq }}^{\alpha}$. In the asymptotics, an approximate relation of the shift of the chemical potential was obtained and included in the derivation [Eq. (81)]. The advantage of a pure parabolic expression is that the chemical potential in Eq. (81) remains exact for arbitrary deviations from the equilibrium. This guarantees the accuracy of the simulation, when benchmarked in conjunction with the sharp interface solution.

On the other hand, as the thermodynamic parameter $A$ does not appear in the asymptotics, its value remains free of choice to the user in the sharp interface limit. However, during the simulation, a small value of $A$ may lead to a substantial loss of volume. This apparently corresponds to the fact that the simulation is confined to a finite interface width and that the volume occupied by the interface remains nonzero. To guarantee sufficient conservation of volume in the following simulations, a value of $A=50 u_{E} / u_{l}^{3}$ was chosen.

\section{Angle measurement}

In deriving the analytical shape of the groove, a boundary condition was employed in terms of the equilibrium angle at the root. Thus, it is necessary to validate whether the dihedral angles are correctly reproduced in the profiles obtained from phase-field simulations. Additionally, certain geometrical features are expected from theory, such as the depth-to-width ratio $d / w$ and the width-to-height ratio $w / h$. From the isoline $\phi_{\delta}=0.5$, the point with the maximum $y$ coordinate determines the position of the groove peak, as depicted in Fig. 7. Moreover, the coordinates of the groove root are determined by finding the intercept of sixth-order polynomial fits at either side of the groove. The slope at the groove root, caused by the polynomial fits, serves to determine the equilibrium angle $\theta$. It should be noted that in the sharp interface theory, the shape was determined only on one side of the GB, when invoking the symmetry of the profile about the root. Contrarily, phase-field simulations require to model the complete groove profile with a finite GB width. As a consequence, the profiles do not meet at a sharp, but rather blunt point at the root, which is just an artifact of the diffuse interface formalism. Therefore, while fitting the profiles with a polynomial, the points corresponding to the region of the triple junction are not taken into account.

\section{RESULTS}

To quantitatively evaluate the temporal behavior of grooving, a comparative study is performed. During the analysis, 


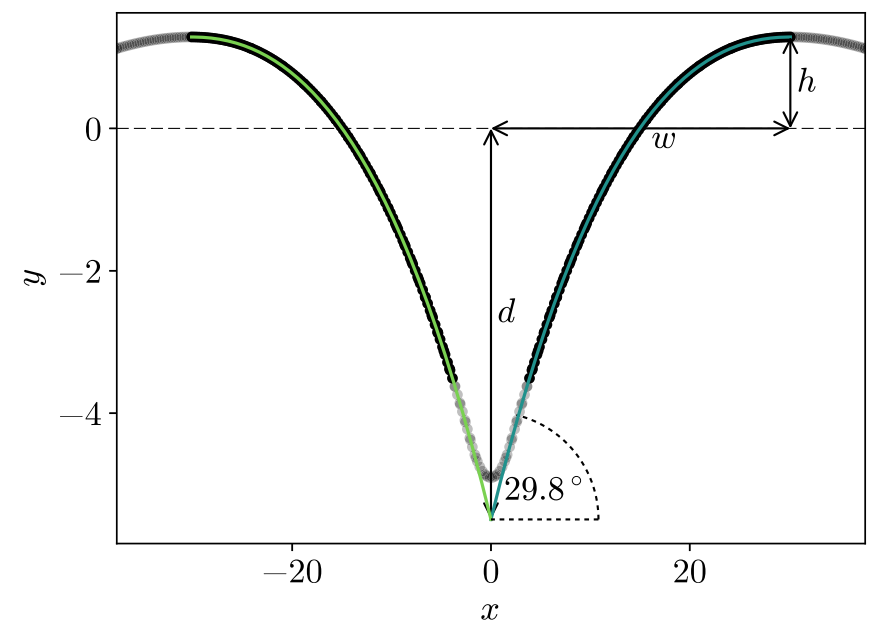

FIG. 7. Measurement of the groove dimensions and equilibrium angle $\theta$ at the groove root, by sixth-order polynomial fits (solid lines) to the isoline $\phi_{\delta}=0.5$ (closed symbols). Points shown as transparent symbols are excluded from the fits.

both the analytical solution for small slopes [19] and the numerical solution for finite surface slopes [20] are considered. Since the phase-field model does not include any small-slope approximation, simulation results are expected to reproduce the fully nonlinear solution. While the asymptotic treatment was restricted to a two-phase system, the applicability of the multiphase model is analyzed for varying triple-junction angles.

First, the features of the groove geometry are analyzed. Hereby, the derived rate constant [Eq. (99)] is tested by appropriately analyzing the widening of the groove. Second, the chemical potential evolution is discussed extensively, and its behavior in the normal and tangential direction is explained in detail. Herein, the validity of the derived Gibbs-Thomson relation is to be verified through Eq. (81). Last, an error analysis is performed to study the interface width dependence of the model.

\section{A. Groove geometry}

The temporal evolution of the groove surface for a thermal groove with equivalent interfacial energies $\left(\gamma_{\mathrm{GB}} / \gamma_{s}=1\right)$ is shown in Fig. 8. The morphological evolution is apparently consistent with the theoretical prediction. Starting from a flat surface $y=0$, at $t=0$ (Fig. 6), a deepening of the groove is observed in the vicinity of the grain-boundary to surface intersection $(x=0)$. Further away from the triple junction, the surface smoothly forms a hill region on both sides. Additionally, a small valley is formed, where the position of the interface lies below $y=0$. Finally, the original flat position is approached. The groove subsequently grows, retaining these geometrical features, while the growth rate decays with time. Furthermore, the groove profiles are seemingly self-similar and independent of time.

According to Eq. (99), the rate constant, expressed with the values given in Table III, evaluates to $B=0.15625 u_{l}^{4} / u_{t}$. The self-similarity of the growth is visualized by plotting subsequent groove geometries in a rescaled coordinate system,

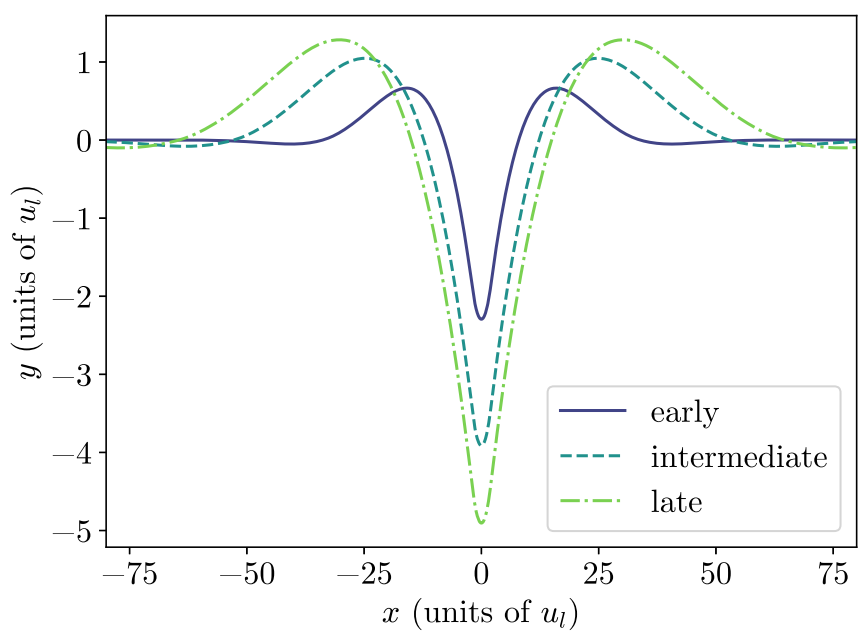

FIG. 8. Simulational groove surface $\left(\phi_{\delta}=0.5\right.$ isoline $)$ for $\gamma_{\mathrm{GB}} / \gamma_{s}=1$, at different times. Early, intermediate, and late times correspond to $t=1.5 \times 10^{4} u_{t}, t=9 \times 10^{4} u_{t}$, and $t=2 \times 10^{5} u_{t}$, respectively.

where both axes are scaled by the factor $1 /(B t)^{\frac{1}{4}}$. The corresponding and appropriately scaled plot from Fig. 8 is shown in Fig. 9. Along with the multiphase-field geometries, the profiles of the sharp interface solutions, considering small and finite slopes, are included as dashed lines. First, the groove geometries of the multiphase-field model (MPF), which are shown as solid lines, at different times, almost perfectly reduce to a single curve. Therefore, the self-similarity of the growth indicates surface diffusion to be the dominant mode of the mass transfer. While the overall agreement of the analytical solution in the small-slope framework and the simulated profile is deemed acceptable, appreciable discrepancies are

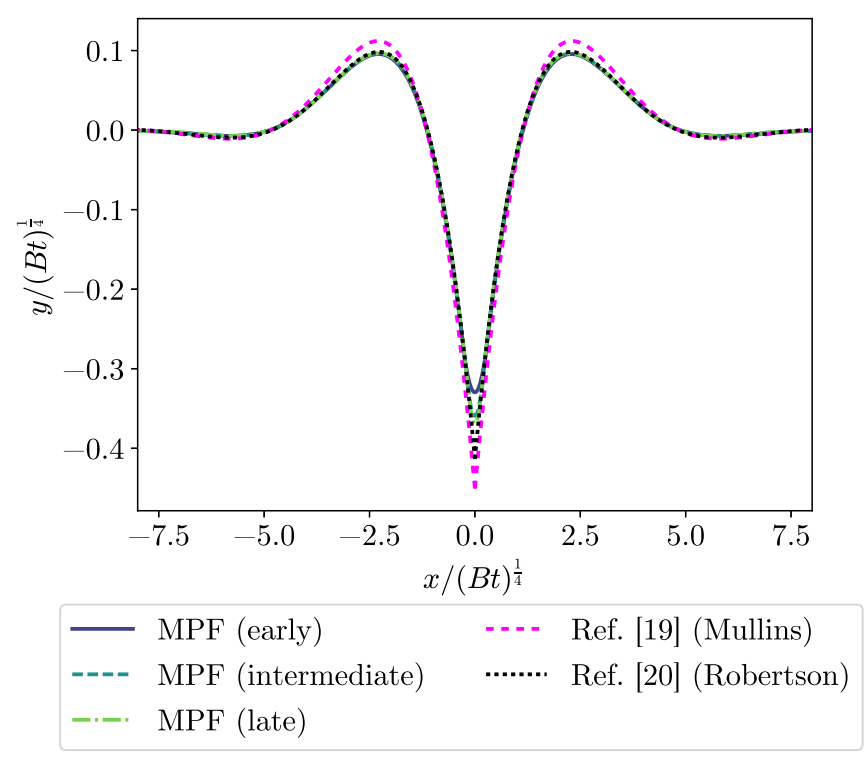

FIG. 9. Evolution of the groove surface for $\gamma_{\mathrm{GB}} / \gamma_{s}=1$, performed at the same times as in Fig. 8, in rescaled coordinates for the proposed multiphase-field model (MPF) and according to Mullins [19] and Robertson [20]. The rescaling is applied, with the value of $B$ being calculated according to Eq. (99). 


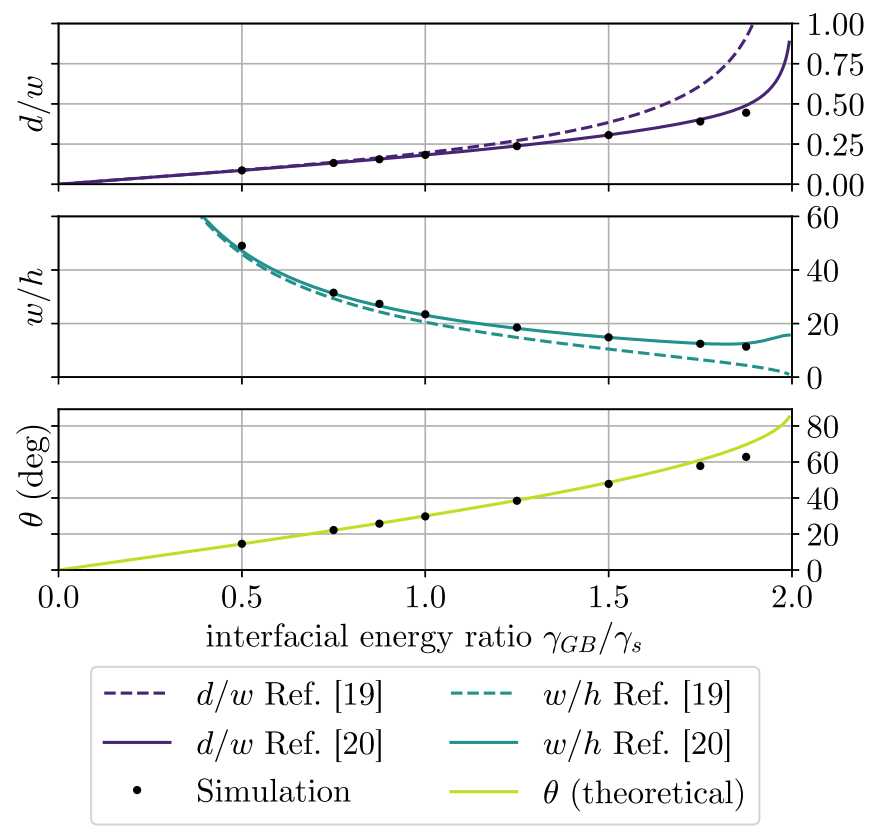

FIG. 10. Characteristic groove dimensions in the final state, obtained from the multiphase-field simulations (closed symbols) and according to the theory in $[19,20]$, as the grain boundary energy is varied. The theoretical triple-junction angle $\theta$ is expressed through Eq. (126).

visible at the groove root and close to the maxima of the grooves. By taking the fully nonlinear numerical solution into account, a much better agreement is found. Therefore, the observed deviation can reasonably be attributed to the limitations of the small-slope approximation.

To study the behavior of the groove growth for a set of triple-junction angles, several simulations with varying grain boundary energy have been performed. Subsequently, in addition to the triple-junction angle, characteristic properties of the groove geometry, namely, the depth-to-width and width-to-height ratios, have been ascertained. Each of the outlined theoretical treatments predict a clear correlation between these properties and the ratio between the grain boundary and the surface energies. In Fig. 10 the resulting relationships from the small-slope approximation and the finite-slope solution are respectively depicted as dashed and solid lines, while the triple-junction angle is plotted by evaluating Eq. (126). Additionally, the measured groove geometries in the final state are shown as solid symbols in Fig. 10. In the upper subplot, a monotonically increasing depth-to-width ratio $(d / w)$ with increasing $\gamma_{\mathrm{GB}} / \gamma_{s}$ is observed, according to theory. As expected, the agreement of both the small-slope and finiteslope grooves is eminently pronounced for small $\gamma_{\mathrm{GB}} / \gamma_{s}$ ratios corresponding to a small triple-junction angle $\theta$. While the phase-field simulations reproduce the prescribed trend of the depth-to-width ratio, the corresponding values apparently follow the fully nonlinear treatment. In contrast to the $d / w$ ratio, the width-to-height ratio decreases with increasing $\gamma_{\mathrm{GB}} / \gamma_{s}$, which is shown in the centered subplot. Again, the simulation results seemingly coincide with the desired interrelation. The good agreement between theory and simulation is evidently attributed to the fact that the triple-junction angle also

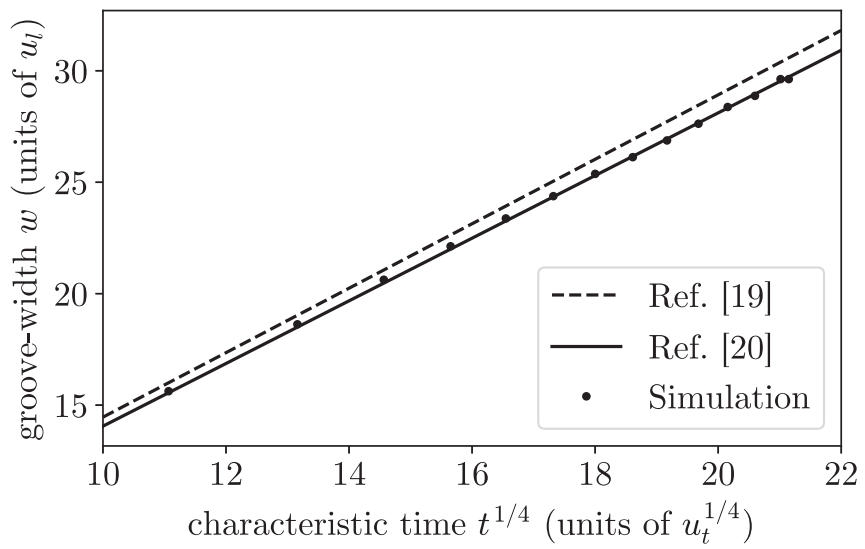

FIG. 11. Groove width $w$, as a function of time, (to the power of $1 / 4$ ) from a multiphase-field simulation at $\gamma_{\mathrm{GB}} / \gamma_{s}=1.5$ (solid symbols) and according to theory $[19,20]$ (dashed and solid lines, respectively). For the theoretical curves, the value of $B$ has been calculated according to the derived formula [Eq. (99)].

follows the theoretical prediction (lower subplot). However, as the grain boundary becomes large $\left(\gamma_{\mathrm{GB}} / \gamma_{s}>1.75\right)$, a small deviation is visible in the plot. While no thorough examination is performed to clarify this discrepancy, one must bear in mind that triple junctions become unstable when the ratio $\gamma_{\mathrm{GB}} / \gamma_{s}$ approaches the value of 2 (which corresponds to $m \rightarrow \infty$ ). Therefore, the groove root has to become infinitely sharp, which clearly shows a practical limitation of the diffuse interface of finite thickness, introduced in the phase-field model.

To a certain extent, all comparative examinations in Fig. 10 rely on the self-similar behavior of the groove profile. As shown in Fig. 8, the characteristic kinetics associated with surface diffusion-governed thermal grooving are related to the self-similarity through the appropriate scaling of the coordinates. Apart from their indirect involvement in scaling, the kinetics of thermal grooving have not been extensively considered yet. To that end, the temporal change in the width of the groove is plotted against the characteristic time $t^{\frac{1}{4}}$, in Fig. 11. For this illustration, the evolution of the system with the interfacial energy ratio of $\gamma_{\mathrm{GB}} / \gamma_{s}=1.5$ is considered.

Although a linear prediction is provided by both sharpinterface treatments, a disparity is observed between two solutions, analogous to previous studies associated with Fig. 10. However, asserting their numerical accuracy, the outcomes of the present approach follow relatively absolute the sharp-interface solution with the finite-slope treatment, as shown in Fig. 11.

\section{B. Chemical potential}

In the asymptotic analysis, a constant chemical potential is obtained in the normal direction of the interface [Eq. (81)]. A first benchmark is to check whether the chemical potential is really constant along the normal direction in the interface. Therefore, the chemical potential field in the vicinity of the triple junction is shown for an early time in Fig. 12. The interfacial region is highlighted by the isolines of the vapor phase field $\phi_{\delta}$. Here the chemical potential along the surface strongly varies from a large positive value at the triple junction 

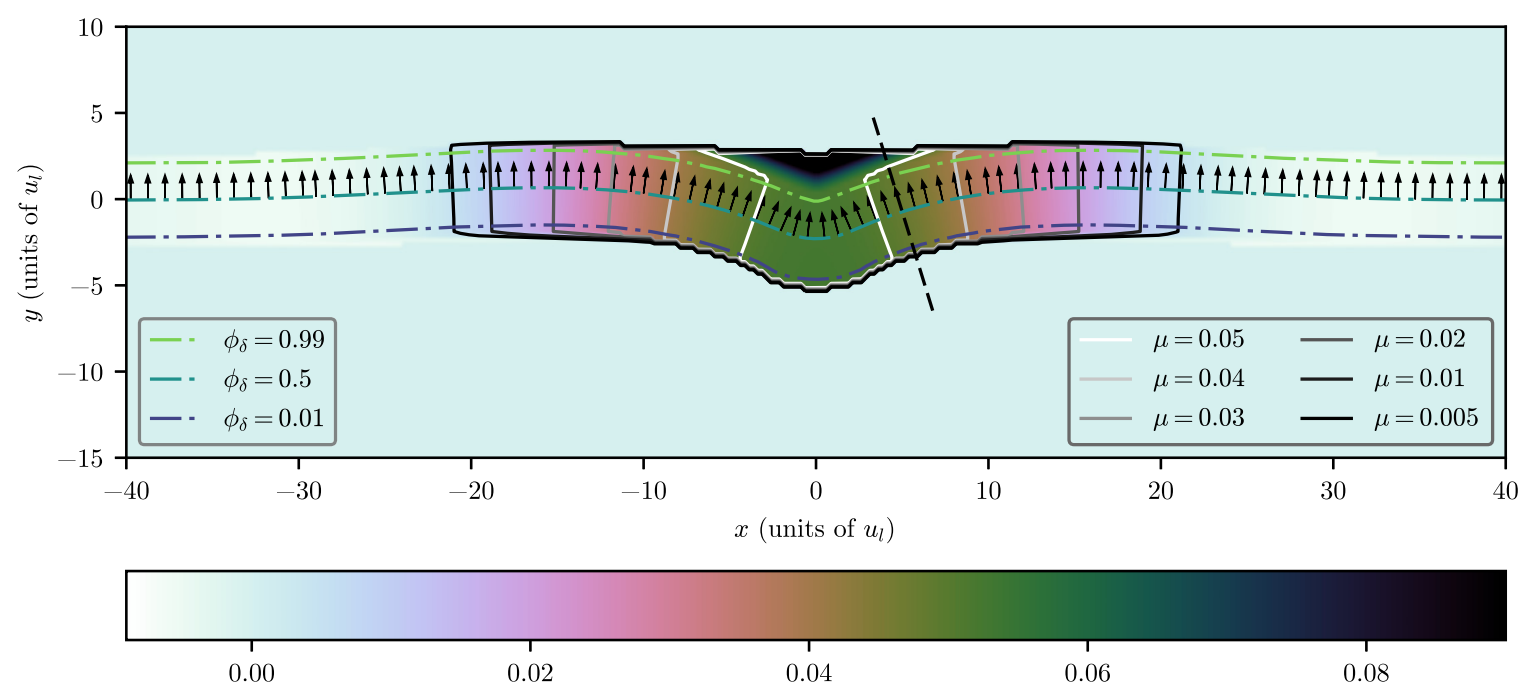

FIG. 12. The chemical-potential field $\mu(\boldsymbol{x})$ (the unit $u_{E} / u_{l}^{3}$ is omitted) at an early time $t=1.5 \times 10^{4} u_{t}$, in a region close to the triple junction $(x=0)$, for equal interfacial energies $\gamma_{\mathrm{GB}}=\gamma_{s}$. The isolines of the chemical potential and the phase field of the vapor phase $\phi_{\delta}$ are superimposed. The arrows indicate the normal vectors on the isoline $\phi_{\delta}=0.5$ and the dashed line represents a normal cross section of the interface.

toward a near-zero value, far away from it. The isolines of the chemical potential, which are strongly aligned along the normal vectors, show that there is a negligible variation of the chemical potential in the normal direction, both close to the triple junction and far away from it. Therefore, the qualitative behavior of the chemical potential in the simulation is consistent with the presented asymptotic analysis.

Furthermore, bilinear interpolation is employed to calculate the chemical potential and the phase field $\phi_{\delta}$ in the normal direction, at an arbitrary point close to the triple junction (dashed line in Fig. 12), which is shown in Fig. 13. The behavior of the chemical potential in the bulk is not treated in the asymptotics and has not been discussed so far. First, it is seen that the chemical potential far away from the interface still fulfills the initial condition $\mu=\mu_{\mathrm{eq}}=0$, which indicates that diffusion occurs only at the interface. Interestingly, the chemical potential field in the bulk does not remain zero all the time, but changes in regions where once was an interface.

Here it is observed that the chemical potential is continuous at the receding side, due to its motion, but discontinuous at the front. Another discontinuity exists in the bulk, which corresponds to the initial flat position of the interface. The existence of such jumps shows the absence of bulk diffusion and the clear decoupling between the interface and the bulk achieved here. In addition to the pronounced match of the phase-field profiles from the simulation and the asymptotics, the position of the interfacial endpoints apparently matches the ones derived here (shown as dashed vertical lines), since the transitions of the interface and the bulk appear at the correct positions.

Through Eq. (81), the value of the chemical potential is related to the curvature and motion of the interface. In the current setup, we have chosen the mobility of the surfaces $m_{\alpha \delta}$ and $m_{\beta \delta}$ in such a way that the effect of the attachment kinetics should be negligible, and thus the term with the interface velocity is deemed insignificant. Hence the approximate relation $\mu=\gamma_{s} \kappa_{s} /\left(c_{S}^{\mathrm{eq}}-c_{\delta}^{\mathrm{eq}}\right)$ is obtained, where the subscript $s$ may be replaced either by $\alpha \delta$ or $\beta \delta$. Note that the equilibrium chemical potential $\mu_{\mathrm{eq}}$ is simply zero for the current choice of free energies. As the theoretical groove shape is known from [20], the value of curvature can be calculated from Eq. (28), given that the time $t$ and the position $x$ are known. By inserting this into the relation above, the theoretical chemical potential in the interface corresponding to the cross section highlighted in Fig. 12 can be calculated. This value is shown in Fig. 13

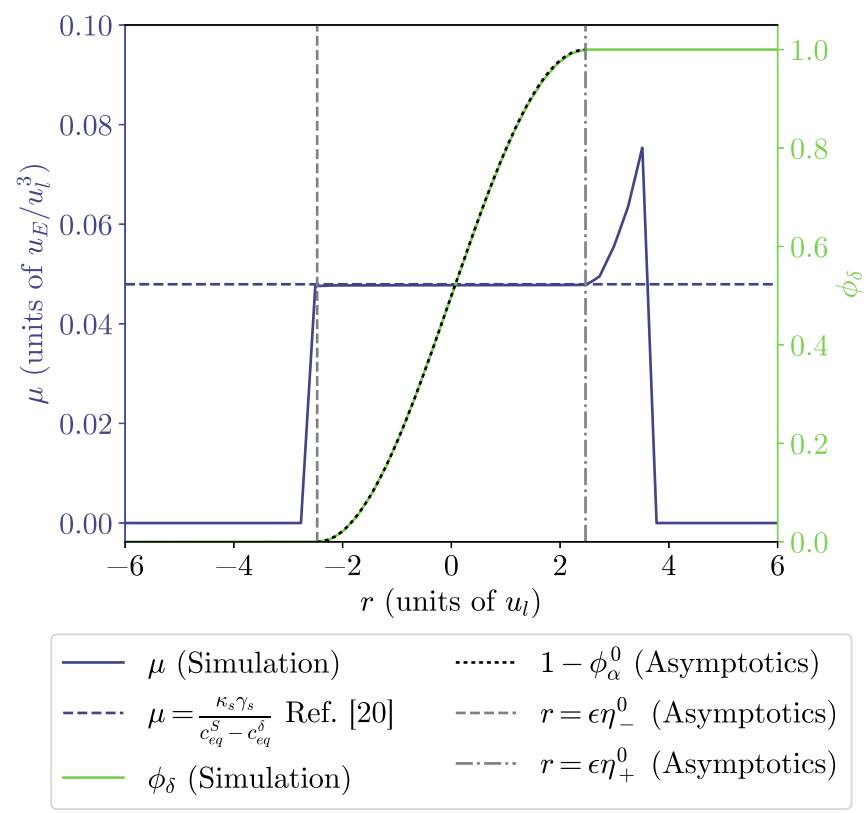

FIG. 13. Chemical potential $\mu$ [purple (black)] and phase field $\phi_{\delta}$ [green (light gray)] across the interface (along the dashed line in Fig. 12). $r$ represents the signed distance from the center of the interface $\left(\phi_{\delta}=0.5\right)$. The horizontally dashed line is the theoretical chemical potential, according to the Gibbs-Thomson effect, calculated from the numerical sharp-interface solution [20], at the same position ( $x$ taken from $\phi_{\delta}=0.5$ intersection) and time. 


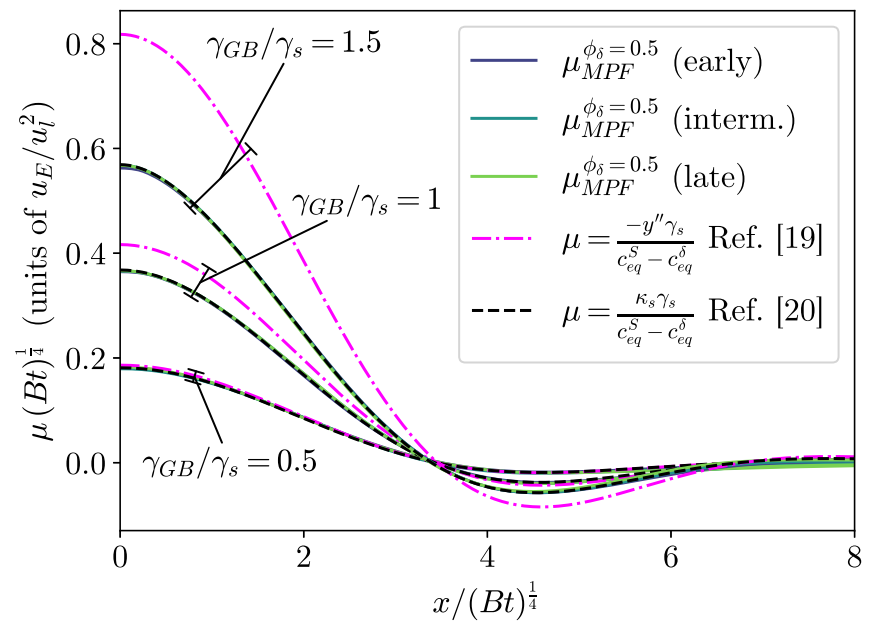

FIG. 14. Scale-invariant chemical potential $\mu(B t)^{1 / 4}$, as a function of the rescaled $x$ coordinate $u=x /(B t)^{1 / 4}$. The solid lines show the chemical potential in the center of the interface (along the isoline $\phi_{\delta}=0.5$ ) for phase-field simulations of varying $\gamma_{\mathrm{GB}} / \gamma_{s}$ ratios, at early [purple (black), $t=1.5 \times 10^{4} u_{t}$ ], intermediate [teal (gray), $t=9 \times 10^{4} u_{t}$ ] and late [green (light gray), $t=2 \times 10^{5} u_{t}$ ] times. The dashed and dash-dotted lines are the theoretical curves, according to the relation obtained in the asymptotics without and with small-slope approximation, respectively.

as a dashed horizontal line. Here an excellent match is found, which shows that the chemical potential in the interface is also in quantitative agreement with the derivation.

To see that the value of the chemical potential matches continuously along the tangential direction, rather than only at a single point, chemical potentials are calculated along the baseline $\phi_{\delta}=0.5$ and are compared with their theoretical counterparts. The corresponding chemical potentials, rescaled in time as $\mu(B t)^{1 / 4}$, to compensate the decay in curvature with time, are shown in a single plot in Fig. 14, for three different simulations and at different times. Note that only the right side of the groove is shown, due to the symmetry. It is seen that the rescaled chemical potential in the interface is nearly invariant in time, when plotted against the dimensionless length $x /(B t)^{1 / 4}$, which is also predicted from the theory. Here the maximum chemical potential (and hence curvature) is assumed at the triple junction $(x=0)$, which is in agreement with both Mullins' and Robertson's solution. Interestingly, even though the surface curvature is not well defined in the region of the triple junction, no significant deviation of $\mu$ is found for small $x$. The chemical potentials using the small-slope approximation [19] are generally of larger magnitude than the ones from the numerically exact solution and simulation, which is particularly pronounced for large $\gamma_{\mathrm{GB}} / \gamma_{s}$ ratios. It is observed that the chemical potential curves from the simulation match best with Robertson's solution, and hence the chemical potentials in the interface are overall consistent with both the sharp interface theory and the asymptotic analysis.

\section{Error analysis}

In the asymptotics, the sharp-interface limit of the model has been derived. In fact, these laws are exactly valid only for
TABLE IV. Absolute $\left(E_{a}\right)$ and relative $\left(E_{r}\right)$ errors for various simulations with different interface widths, at a late time $t=1.95 \times$ $10^{5} u_{t}$. The results for two different representations of the groove surface, $\phi_{\delta}=\phi_{\beta}$ and $\phi_{\delta}=0.5$, are presented.

\begin{tabular}{lccccc}
\hline \hline$\epsilon\left(u_{l}\right)$ & $\Delta x\left(u_{l}\right)$ & $E_{a}^{\phi_{\delta}=\phi_{\beta}}$ & $E_{r}^{\phi_{\delta}=\phi_{\beta}}(\%)$ & $E_{a}^{\phi_{\delta}=0.5}$ & $E_{r}^{\phi_{\delta}=0.5}(\%)$ \\
\hline 2.0 & 0.125 & 0.000554 & 0.356 & 0.00485 & 3.12 \\
2.0 & 0.25 & 0.00224 & 1.44 & 0.00532 & 3.42 \\
3.0 & 0.1875 & 0.00122 & 0.783 & 0.00886 & 5.70 \\
3.0 & 0.25 & 0.00152 & 0.976 & 0.00889 & 5.71 \\
4.0 & 0.25 & 0.00218 & 1.40 & 0.0136 & 8.74 \\
4.5 & 0.25 & 0.00203 & 1.31 & 0.0163 & 10.5 \\
5.0 & 0.25 & 0.00357 & 2.30 & 0.0189 & 12.2 \\
5.5 & 0.25 & 0.00413 & 2.65 & 0.0217 & 14.0 \\
6.0 & 0.25 & 0.00568 & 3.65 & 0.0246 & 15.8 \\
6.5 & 0.25 & 0.00700 & 4.50 & 0.0276 & 17.7 \\
7.0 & 0.25 & 0.00851 & 5.47 & 0.0307 & 19.7 \\
7.5 & 0.25 & 0.0102 & 6.54 & 0.0338 & 21.7 \\
8.0 & 0.25 & 0.0120 & 7.73 & 0.0370 & 23.8 \\
10.0 & 0.25 & 0.0210 & 13.5 & 0.0503 & 32.3 \\
12.0 & 0.25 & 0.0324 & 20.8 & 0.0641 & 41.2 \\
14.0 & 0.25 & 0.0460 & 29.6 & 0.0781 & 50.2 \\
16.0 & 0.25 & 0.0618 & 39.7 & 0.0924 & 59.4 \\
\hline \hline
\end{tabular}

an interface of vanishing thickness. Consequently, a deviation is expected for nonzero interface thicknesses. To investigate the error of the model as a function of interface thickness, thermal grooving simulations were conducted with varying values for $\epsilon$. It is important to note that small interface widths require a high spatial resolution, because the ratio between the interface width and the grid spacing $(\epsilon / \Delta x)$, and hence the number of grid points in the interface, should not be too small. As a consequence, simulations at a low interface width can become very costly. To keep the computational effort small, the interface widths are increased rather than decreased, starting from the standard choice $\epsilon=2 u_{l}$. Generally, the grid spacing has been kept constant, and hence higher interface widths are represented by more grid points in the interface. This ensures that a possible error increase is related to the model and not to the limitations of the discretization.

To quantify the error, $L^{2}$ norms of the groove profile are utilized in rescaled coordinates, $u=x /(B t)^{1 / 4}$ and $z(u)=$ $y\left(u(B t)^{1 / 4}, t\right) /\left[m(B t)^{1 / 4}\right]$. Accordingly, the quantity $\|z\| \equiv$ $\sqrt{\left\{\int_{0}^{u_{\max }}\left[z_{R}(u)\right]^{2} d u\right\} / u_{\max }}$ is introduced, where $z_{R}(u)$ is the numerically exact solution from Robertson [20]. Taking the rescaled profile from the simulation $z_{\mathrm{MPF}}(u)$, an absolute error is defined as $E_{a} \equiv \sqrt{\left\{\int_{0}^{u_{\max }}\left[z_{\mathrm{MPF}}(u)-z_{R}(u)\right]^{2} d u\right\} / u_{\max }}$, and the relative error reads as $E_{r} \equiv E_{a} /\|z\|$. In the following, a cutoff is chosen at $u_{\max }=8$, so as to disregard the region of a negligible variation of $z$. All simulations are performed at equal interfacial energies $\left(\gamma_{\mathrm{GB}} / \gamma_{s}=1\right)$ and the corresponding value of the $L^{2}$ norm from the sharp interface solution constitutes $\|z\| \approx 0.1556$. The corresponding errors of the performed simulations are presented in Table IV. Two different representations of the surface are studied, which differ only in the triple-junction region, namely, $\phi_{\delta}=\phi_{\beta}$ and the isolines $\phi_{\delta}=0.5$. Note that the isoline $\phi_{\delta}=0.5$ does not meet the point $\phi_{\alpha}=\phi_{\beta}=\phi_{\delta}=1 / 3$, which may be seen as the 


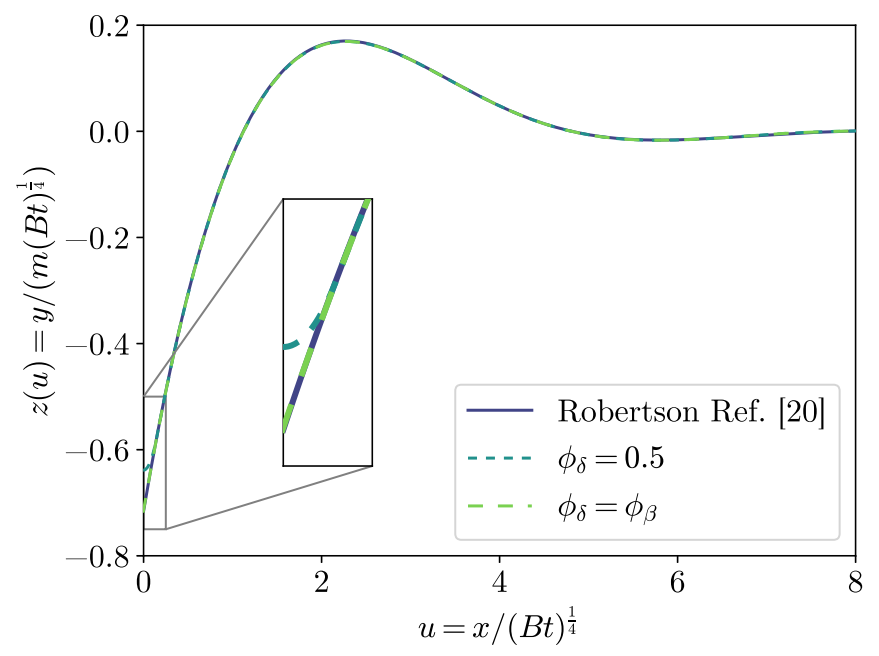

FIG. 15. Rescaled groove profiles from two different representations of the groove surface, at a high resolution $\left(\epsilon=2 u_{l}, \Delta x=\right.$ $\left.0.125 u_{l}\right)$ and at a late time $t=1.95 \times 10^{5} u_{t}$. Note the blunt in the isoline $\phi_{\delta}=0.5$, which is absent in the curve $\phi_{\delta}=\phi_{\beta}$. The sharp interface solution from [20] is shown additionally.

center of the triple junction, whereas the other representation does. Therefore, a blunt is absent in the isoline $\phi_{\delta}=\phi_{\beta}$ (see Fig. 15 for a visual impression). Consequently, the errors $E_{a / r}^{\phi_{\delta}=\phi_{\beta}}$ from this representation, listed in Table IV, are generally smaller than those of the isoline $\phi_{\delta}=0.5$ (pertaining to $E_{a / r}^{\phi_{\delta}=0.5}$ ), which is especially pronounced for small interface widths. At the standard resolution $\Delta x=0.25 u_{l}$, errors taking the isoline $\phi_{\delta}=0.5\left(E_{a / r}^{\phi_{\delta}=0.5}\right)$ are increasing monotonically with $\epsilon$, as desired. Here the smallest achieved relative error at $\epsilon=2 u_{l}$ is about $3.5 \%$. However, taking the errors of the other representation into account, it is seen that $E_{a / r}^{\phi_{\delta}=\phi_{\beta}}$ does not converge properly for small interface widths. For a smaller $\epsilon$, errors show oscillations and an error increase is observed from $\epsilon=3 u_{l}$ to $\epsilon=2 u_{l}$. To check whether such an error increase is related to the model or to the limitations of the discretization, two simulations have been performed at a higher resolution. Here the ratio between the interface width and the grid spacing is kept identical to the case of $\epsilon=4 u_{l}$, as $\epsilon / \Delta x=16$, which results in $\Delta x=0.125 u_{l}$ and $\Delta x=0.1875 u_{l}\left(N_{x}\right.$ and $N_{y}$ are increased so as to represent the same region), for $\epsilon=2 u_{l}$ and $\epsilon=3 u_{l}$, respectively. These values are included in Fig. 16, to study the scaling of the error $E_{r}^{\phi_{\delta}=\phi_{\beta}}$ with $\epsilon$. By taking only the high-resolution simulations into account (closed symbols), it is observed that the error now increases monotonically (apart from small residual oscillations). Therefore, the error at $\epsilon=$ $2 u_{l}$ and at lower resolutions is dominated by the discretization. We think that this is mainly attributed to the shallow geometry of the groove, which spans only a small number of grid points in height and hence is prone to discretization errors. Therefore, the choice of $\Delta x$ is not quite optimal, since $\epsilon=2 u_{l}$ and $\Delta x=0.25 u_{l}$ are the standard combination in the current document. Most of the error, visible so far, is probably caused by the limited discretization. However, as the relative error is estimated to be around two percent, the match with the sharp-interface solution is held acceptable.

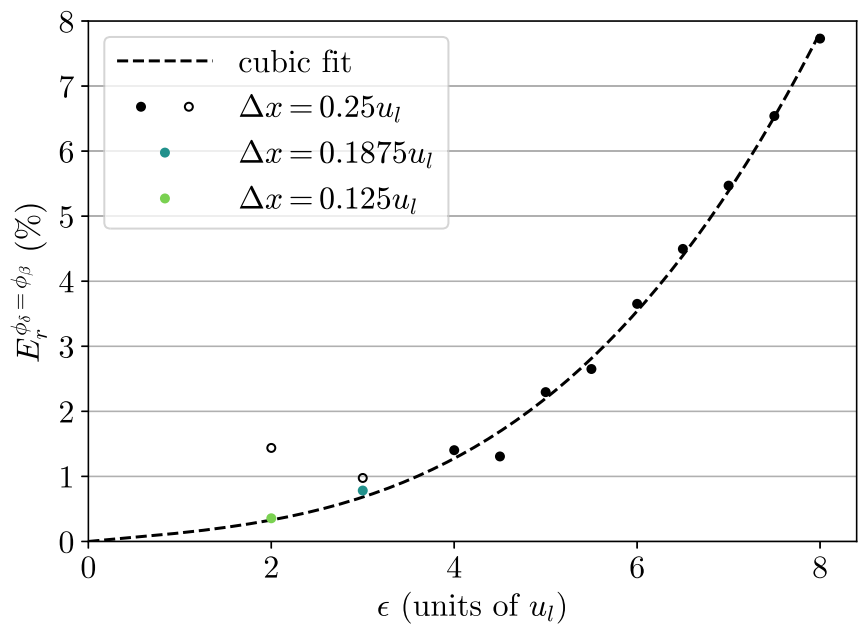

FIG. 16. The relative error between the phase field and sharp interface solutions for simulations at small interface widths (values tabulated in Table IV). The symbols represent the errors obtained from the phase-field simulations, whereas the dashed line shows a polynomial fit to the closed data points. For the fit, the open symbols are not taken into account. The colored (grayish) symbols refer to simulations at a higher resolution.

Finally, a polynomial fit and a power fit have been calculated from the solid symbols in Fig. 16. Using the least squares method, the polynomial fit writes as $E_{r}^{\phi_{\delta}=\phi_{\beta}} \approx a^{1} \epsilon+$ $a^{2} \epsilon^{2}+a^{3} \epsilon^{3}$, where the coefficients are found to be $a^{1}=$ $0.13 \% / u_{l} \pm 0.14 \% / u_{l}, \quad a^{2}=-0.01 \% / u_{l}^{2} \pm 0.05 \% / u_{l}^{2} \quad$ and $a^{3}=0.015 \% / u_{l}^{3} \pm 0.004 \% / u_{l}^{3} \cdot u_{l}$ denotes the unit of length. As can be seen in Fig. 16, the fit matches apparently well with the data points. An additional fit reveals the order of convergence in the shown interval $E_{r}^{\phi_{\delta}=\phi_{\beta}} \approx a_{p} \epsilon^{n}$ to be approximately superquadratic, as the found exponent is larger than two $\left(n=2.67 \pm 0.09, a_{p}=0.030 \% / u_{l}^{n} \pm 0.005 \% / u_{l}^{n}\right)$, which also corresponds to the third-order polynomial term $a^{3} \epsilon^{3}$, dominant in most of the interval. For a smaller $\epsilon$, the linear and quadratic term become more dominant, and the speed of convergence is decreased. This is consistent with the fact that the recovered laws are correct up to the zeroth order in $\epsilon$.

\section{DISCUSSION}

\section{A. Freedom to choose a mobility function}

In conventional Cahn-Hilliard-based surface-diffusion models, a single order parameter serves the dual role of tracking the solute composition as well as imprinting capillarity into the system, while separate phase and composition variables offer a clear decoupling of mass transport and interface migration, in the current model. As demonstrated in the current work, this provides the freedom to choose a particular form of degenerate mobility function $g(\phi)$ (Table I). In contrast, the mobility function $M g(c)$ in a Cahn-Hilliard model is written in terms of the concentration $c$. Since this function is directly included in the evolution equation of the same variable, the solution strongly depends on the combination of the particular form of $g(c)$ and on the corresponding potential of well or obstacle type, as demonstrated in $[44,45]$, 
which in turn may lead to an unwanted volume-diffusion term. Additionally, the use of stabilizing functions or tensorial mobility is not a requirement in the present model. However, the efficiency of scalar and tensorial mobilities, in the context of coupled second-order conservative and nonconservative systems of equations, remains to be evaluated and is reserved for a future investigation. As the underlying grand-potential model was successfully extended to multiphysics (e.g., mechanics $[89,90])$, the current surface-diffusion model suits an easy amplification in future applications as well.

\section{B. Computational cost}

A common construction in the Cahn-Hilliard-based models is the use of a fourth-order differential equation. The presence of a fourth-order equation, in contrast to the second-order conservative and nonconservative coupled equations, introduces a number of computational complexities. First, the discretization of two compounded Laplacians increases the computational cost significantly. Second, since an explicit scheme is commonly used for time integration, a severe restriction, due to the CFL condition on the time step size $\Delta t$, exists, which, on the one hand, scales with an exponent of four $\left(\Delta x^{4}\right)$, with the spatial discretization $\Delta x$. On the other hand, a scaling with a smaller exponent $\left(\Delta x^{2}\right)$ is expected in the present model, since only second-order equations are solved. In the current model, an additional advantage of the applied obstacle-type potential is that the system of equations restricts the evaluation of the governing equations to the interfacial region. However, a detailed comparison of the computational efficiency of Cahn-Hilliard-based models and the present model is not considered in the current paper and may be tackled in future works.

\section{Gibbs-Thomson relation}

The present model manages both coupled motion by surface diffusion and attachment kinetics, as well as the corresponding Gibbs-Thomson effect in the sharp interface limit. Nevertheless, the derived Gibbs-Thomson relation only approximately equals the sharp interface limit [Eq. (27)] for a pure substance, which is usually exactly recovered (either as a zeroth- or first-order correction of $\omega$ ) in conventional Cahn-Hilliard surface diffusion models. One may argue that this is a limitation of the current model. However, as it turned out, the obtained relation [Eq. (79)] captures all nonlinearities for a binary system [Eq. (A7)], involved in a more general Gibbs-Thomson condition. It is deduced that the current model provides a more realistic treatment, given the correct free energies $f^{\alpha}$ of the material system at hand. On the other hand, the governing equation (95) includes bulk compositions. Therefore, the results of the current model are dependent on the initial condition. This, however, is not an artifact of the phase-field model, but is required to fulfill the mass balance at the interface (see Appendix B). Mathematically, however, the current model will reduce to the simple set of equations, if the composition is fixed in the bulk, which can be achieved in the limit $\partial c^{\alpha} / \partial \mu \rightarrow 0 \forall \alpha$, by choosing adequate free energies $f^{\alpha}$.
A possible way of approaching the pure substance (unary) case is to keep simple parabolic free energies, but making the sharpness of the parabolas dependent on $\epsilon$ and setting $c_{\mathrm{eq}}^{\alpha}$ to zero and unity, for the vapor and solid phase, respectively. In this modification of the model, the factor $A$ in Eq. (138) is replaced by $A=\tilde{A} / \epsilon$, where $\tilde{A}$ is a positive constant, which ensures that in the sharp interface limit, bulk compositions remain at exactly zero and unity even when shifts in the chemical potential occur. This is reflected in no-flux boundary conditions instead of convective ones (cf. Appendix C 2). This is attributed to the fact that, at lowest order in $\epsilon$, the phase-inherent compositions, which write now as $c^{\alpha}(\epsilon)=c_{\text {eq }}^{\alpha}+\epsilon \mu /(2 \tilde{A})$, reduce to the equilibrium values. Higher orders $\left(A=\tilde{A} / \epsilon^{n}, n>1\right)$ are also possible and should lead to a stronger suppression of composition shifts as $\epsilon$ is reduced. This, as it turns out, is in fact an advantage in contrast to the existing well-potential Cahn-Hilliard models. There the order of $\epsilon$ at which, analogously, the wells in the well potential become sharper is fixed to maintain a finite interfacial width. However, this detail of the models cannot be treated rigorously without going beyond a sharp-interface analysis, and hence is left as a possible future task.

A final objection could be the following: Even though the forms of the sharp-interface Eqs. (35) and (36) for a pure substance may be recovered with a reassignment, philosophywise the current construction cannot be claimed to be a phase-field model of surface diffusion in pure solids as it is based on the grand-potential model, which is an alloy-specific formulation. It is pointed out that instead of starting with an alloy terminology and doing a mere remapping in the end, one can start with reinterpreting the various quantities right from the beginning as presented in Appendix A 3. Of course, in such a depiction, compression is exclusively coupled to an exchange of vacancies, and is thus incomplete, it might still be claimed that our formulation can also be supposed as a model for pure systems. Moreover, for an almost incompressible solid that is usually considered in the sharp-interface theories, this depiction becomes satisfactory.

\section{Chemical potential profile}

\section{Across the interface}

An important property of the current model is the constancy of the chemical potential in the normal direction. In fact, this point has also been mentioned in the work of Amirouche et al. [73], where a similar model was employed. There, in contrast to the current work, difficulties in recovering the Gibbs-Thomson effect and a strongly varying chemical potential in the interface were observed. The authors argued that this probably has to do with a so-called breakdown of locality. However, one of the major differences between the two models is the utilization of a well-type potential in their work. Hence, the equations derived here are not directly applicable. Moreover, no asymptotic analysis is available for the model used in the study above. Thus, we can only guess what may have caused the inconsistency. One possibility is that the chemical potential may not tend to a normally constant profile as interface width is tending to zero. If, on the other hand, it turns out that a constant zeroth-order chemical potential can 
also be derived on the basis of a well potential, the conclusion would be that the interfacial width was not chosen small enough to operate appropriately in the sharp interface limit. Accordingly, the contributions of the higher-order terms, $\mu^{1}$ and $\mu^{2}$, which much likely are not constant, may become important. A study on interfacial width, as done in the current work, might help to clarify the confusions regarding the breakdown of locality. Furthermore, in contrast to the current paper, small, but nonvanishing diffusivities are applied in the work of Amirouche et al. It is much likely that this will not drastically change the picture. However, it still is not answered thoroughly enough and clearly deserves further attention.

In contrast to the chemical potential in the interface, the role of the bulk chemical potential has not yet been discussed in the current paper, which will be done in the following.

\section{In the bulk}

It was observed that the chemical potential in the bulk is overwritten by the chemical potential in the interface, due to the absence of bulk diffusion, and thereafter remains invariant (see Fig. 13). A consequence of this is the existence of discontinuities in the chemical potential. However, it is still unclear whether the chemical potential is truly discontinuous at the front, or whether it is only varying rapidly in a small, but finite transition region (see Appendix E for more details). Please note additionally that the concept of vanishing diffusivities may be seen as a simplification, in a physical perspective, since some tiny amount of residual diffusion does probably always exist in any real bulk material. In such a situation, the discontinuities might disappear to form a steep, but well-defined chemical-potential profile at the transition from interface to bulk. When inspecting the evolution equation of the chemical potential [Eq. (18)] for the bulk case $(M=0)$, it should not evolve, since the phase field is also invariant in the bulk. This way, the observation of an invariant chemical potential in the bulk is consistent with the nature of the evolution equation. However, this also implies that there is no possibility to rearrange the value of the chemical potential at a certain point, once the interface has moved away from it. As a consequence, the chemical potential in the bulk is history-dependent, and its value is determined by the chemical potential of the most recent interfacial sweep. In other words, the interface leaves its current chemical potential behind. In fact, this has some serious consequences, because, in view of the interface, it leads to convective fluxes entering or leaving it, once it moves. This corresponds to a deposition of mass in the bulk. Such terms do not exist in the sharp-interface theory, which considers pure substances. This is attributed to the fact that the bulk compositions in the pure substance treatment are a priori fixed at zero and unity. We point out that this is of course not true for an alloy where shifts in compositions occur simultaneously with shifts in chemical potentials. Hence, the current model seems to successfully render this behavior as well.

Nevertheless, the question arises whether the motion of a boundary may alter the composition in the bulk, which is observed here. Since the atoms have to rearrange themselves at the boundary, once it moves, this is at least regarded as a possibility. There is some evidence that grain boundaries will leave an increased vacancy content behind [125-128]. However, the authors in [128] conclude that this is due to the excess volume, associated with the GB, and not due to a Gibbs-Thomson effect. Therefore, the question remains to be answered whether bulk compositions for an interphase boundary are similarly changed, due to capillarity.

\section{CONCLUSIONS}

A multiphase-field model is formulated to incorporate mass transport by surface diffusion into the grand-potential framework. Since the diffusion equation remains to be of second order, and an obstacle potential is applied, the proposed model is fundamentally different and cost-effective compared to the conventional Cahn-Hilliard-based formulations. The discussed model extension is regarded to be promising for the application to a wide range of materials, since the composition is not required to assume an a priori fixed value in the bulk. An asymptotic analysis is performed for a binary two-phase system, in the absence of volume diffusion. A procedure of Cahn et al. is adopted [22] to restrict the analysis solely to the interfacial region. A combination of surface diffusion and surface attachment kinetics [21] is retrieved as the governing law in the sharp interface limit. The asymptotic analysis also demanded that the chemical potential be constant across the interface as its thickness tends to zero. Moreover, the retrieved Gibbs-Thomson effect is consistent with a general law for a binary system, which reduces to the simple well-known unary relation, in the case of a high immiscibility. As an important outcome, an analytical expression is derived for the governing rate constant, in terms of the model parameters. Furthermore, higher-order corrections to the phase-field profile are obtained in the analysis, which are carefully validated with simulation results on a cylindrical particle. Subsequently, a systematic simulation study is employed on thermal grooving under surface diffusion. In the comparative study, theoretical results for small-slope [19] and finite-slope [20] grooves are considered. The simulation results agree well with the theoretical results, both qualitatively and quantitatively. The latter is demonstrated by measuring the deviation between the theoretical and the simulated groove profiles by means of $L^{2}$-norms. The scaling of the errors with respect to interfacial thickness is also discussed. It was observed that the kinetics can be correctly quantified by the derived rate constant. Additionally, the grooving simulations also revealed that the chemical potential is indeed constant in the normal direction even close to the triple-junction and also follows the derived quantitative relation. The model is formulated to enable a combination of bulk and surface diffusion. However, the influence of an additional bulk flux is not discussed. The generalization of the asymptotic derivation for concomitant surface and volume diffusion is reserved for a possible future work.

\section{ACKNOWLEDGMENTS}

The authors gratefully acknowledge financial support by the German Federal Ministry for Economic Affairs and Energy (BMWi) within the project KerSOLife100 under Funding No. 03ET6101A. P.W.H. thanks Patricia Haremski, Anika Marusczyk, and Matthias Wieler from Robert Bosch $\mathrm{GmbH}$ 
for many interesting discussions, Andreas Reiter for his help in implementing the model, and Andreas Prahs for pointing out the right literature. Further, this work contributes to the research performed at CELEST (Center for Electrochemical Energy Storage Ulm-Karlsruhe) and was partially funded by the German Research Foundation (DFG) under Project ID 390874152 (POLiS Cluster of Excellence). Parts of the asymptotics was funded through the Gottfried-WilhelmLeibniz project NE 822/31-1.

\section{APPENDIX A: DEPARTURE OF EQUILIBRIUM CHEMICAL POTENTIAL UNDER CAPILLARITY}

\section{Unary systems}

Let us consider a unary nonreacting system, i.e., one which contains only a single sort of atoms or molecules which cannot decompose to form a new sort of species. The system comprises an ambient phase $\beta$, which is in contact with the surroundings, and an enclosed spherical particle of phase $\alpha$. The sought relation between the chemical potential $\tilde{\mu}$ and the curvature $\kappa$ (twice the mean curvature) is specified in $[98$, p. 434] as

$$
\frac{\partial \tilde{\mu}}{\partial \kappa}=\frac{\gamma_{\alpha \beta} V_{m}^{\alpha} V_{m}^{\beta}}{V_{m}^{\beta}-V_{m}^{\alpha}} .
$$

Note that we have switched the naming $\alpha$ and $\beta$ of the phases with respect to the textbook. $V_{m}^{\alpha}$ and $V_{m}^{\beta}$ denote the molar volumes of both phases, and $\gamma_{\alpha \beta}$ represents the interfacial energy between them. A direct evaluation of the chemical potential shift can be obtained by integrating the above equation. If the system is compressible, the molar volume may vary as a function of pressure (which varies with curvature), and hence the right-hand side might well be dependent on $\kappa$. Giving an explicit formula therefore involves taking into account the physical properties of the system at hand. Only an approximation might be applied for condensed matter in contact with its vapor. For such a system, the conditions $V_{m}^{\beta} \gg V_{m}^{\alpha}$ and $V_{m}^{\alpha}$, which are almost independent of pressure (low compressibility of the condensed phase), might be applied. In this limit, one receives

$$
\tilde{\mu} \approx \tilde{\mu}(\kappa=0)+V_{m}^{\alpha} \gamma_{\alpha \beta} \kappa .
$$

This is the Gibbs-Thomson relation used in the Mullins theory [Eq. (27)]. In the following, based on DeHoff's derivation, the relations for a binary two-phase system will be presented (the same problem treated in the asymptotics here).

\section{Binary systems}

All derivations are performed in [98, p. 435 ff.]. We will only compile the equations to end up at the desired relations. The only assumptions imposed by the author are that the ambient pressure and temperature of the system are equal before and after the shift from curvature.

The first equation relates the composition of the second component in the ambient phase to curvature, which writes as

$$
\frac{\partial c_{2}^{\beta}}{\partial \kappa}=\gamma_{\alpha \beta} c_{2}^{\beta} \frac{c_{1}^{\alpha} V_{m, 1}^{\alpha}+c_{2}^{\alpha} V_{m, 2}^{\alpha}}{\tilde{\mu}_{12}^{\beta}\left(c_{2}^{\beta}-c_{2}^{\alpha}\right)} .
$$

Here $c_{i}^{\alpha}$ and $c_{i}^{\beta}$ denote the compositions in either phase of the $i$ th component and are therefore unitless. The same notion is also used in the current paper. Accordingly, the sum conditions $c_{1}^{\beta}+c_{2}^{\beta}=1$ and $c_{1}^{\alpha}+c_{2}^{\alpha}=1$ are satisfied, from which follows $c_{2}^{\beta}-c_{2}^{\alpha}=c_{1}^{\alpha}-c_{1}^{\beta}$. $V_{m, i}^{\alpha}$ denotes the molar volume of the $i$ th component in the enclosed phase. $\tilde{\mu}_{12}^{\beta} \equiv\left(\partial \tilde{\mu}_{1}^{\text {ind }} / \partial c_{2}\right)^{\beta}$ represents the dependence of the chemical potential of the first component on the composition of the second component in the ambient phase, and thus is a chemical property of that phase. Therefore, the shift in the chemical potential of the first component can be written as

$$
\frac{\partial \tilde{\mu}_{1}^{\text {ind }}}{\partial \kappa}=c_{2}^{\beta} \gamma_{\alpha \beta} \frac{c_{1}^{\alpha} V_{m, 1}^{\alpha}+c_{2}^{\alpha} V_{m, 2}^{\alpha}}{c_{1}^{\alpha}-c_{1}^{\beta}} .
$$

According to the Gibbs-Duhem equation, a similar relation can be obtained for the chemical potential of the second component, which reads as

$$
\frac{\partial \tilde{\mu}_{2}^{\text {ind }}}{\partial \kappa}=-c_{1}^{\beta} \gamma_{\alpha \beta} \frac{c_{1}^{\alpha} V_{m, 1}^{\alpha}+c_{2}^{\alpha} V_{m, 2}^{\alpha}}{c_{1}^{\alpha}-c_{1}^{\beta}} .
$$

This is already as far as we can go generically. The similarity between the equations allows one to introduce the quantity $\tilde{\mu}_{1} \equiv \tilde{\mu}_{1}^{\text {ind }}-\tilde{\mu}_{2}^{\text {ind }}$, which provides the difference in the chemical potentials between the two components and turns out to be sufficient to track the chemical condition of the system. Then the following relation is obtained:

$$
\frac{\partial \tilde{\mu}_{1}}{\partial \kappa}=\frac{\partial \tilde{\mu}_{1}^{\text {ind }}}{\partial \kappa}-\frac{\partial \tilde{\mu}_{2}^{\text {ind }}}{\partial \kappa}=\gamma_{\alpha \beta} \frac{c_{1}^{\alpha} V_{m, 1}^{\alpha}+c_{2}^{\alpha} V_{m, 2}^{\alpha}}{c_{1}^{\alpha}-c_{1}^{\beta}} .
$$

This is the final equation, relating the chemical potential difference of a binary system to curvature in a generic form.

For a simple system, an interpretation of the relation is given in the following. Given that the molar volumes of each component in phase $\alpha$ are equal $\left(V_{m}^{\alpha}=V_{m, 1}^{\alpha}=V_{m, 2}^{\alpha}\right)$, which might be an approximation for substitutional mixing, the above formula simplifies to

$$
\left.\frac{\partial \tilde{\mu}_{1}}{\partial \kappa}\right|_{V_{m, 1}^{\alpha}=V_{m, 2}^{\alpha}}=\frac{V_{m}^{\alpha} \gamma_{\alpha \beta}}{c_{1}^{\alpha}-c_{1}^{\beta}} .
$$

Furthermore, if the phases are incompressible $\left(V_{m}^{\alpha}\right.$ is independent of $\kappa)$ and nearly immiscible $\left(\tilde{\mu}_{12}^{\alpha}\right.$ and $\tilde{\mu}_{12}^{\beta}$ are large, such that the change in composition in either phase is negligible), one may explicitly write

$$
\tilde{\mu}_{1} \approx \tilde{\mu}_{1}(\kappa=0)+\frac{V_{m}^{\alpha} \gamma_{\alpha \beta} \kappa}{c_{1}^{\alpha}(\kappa=0)-c_{1}^{\beta}(\kappa=0)} .
$$

This is also the expression given in the Appendix of [78], with the difference that the necessary assumptions are explicitly outlined here.

An interesting observation now is made in comparison with Eq. (A2): The chemical behavior under capillarity, of a single condensed substance in contact with its vapor, is qualitatively identical to a binary system under isobaric conditions, comprising phases which are incompressible and immiscible. The only difference is that in a binary system, the switch from a single chemical potential to a potential difference has to be made. A quantitative difference is only observed in the prefactor, which contains an additional composition difference in 
the denominator for a binary system. If the phases represent pure substances, we might simplify $c_{1}^{\alpha} \approx 1$ and $c_{1}^{\beta} \approx 0$ even further, and Eqs. (A2) and (A8) become quantitatively identical.

\section{Connection between a unary and a binary system}

Even though a solid-vapor system of a single component is usually treated as a unary system and delineated through phase-inherent molar volumes, a connection between a compressible unary and an incompressible binary system might be established through vacancies. This way, a volumetric portion of the vacuum is assigned to a hypothetical particle, the so-called vacancy. If the size of the volumetric portion is chosen equal to that occupied by an atom in the solid, the assumption of equal molar volumes remains valid. Therefore, the vapor might easily be distinguished from the solid composition-wise. The composition then represents the mole fraction of the atoms (with respect to the moles of atoms plus vacancies), which is high in the solid and low in the vapor (where most of the volume is occupied by vacancies). This is the concept used here to distinguish between vapor and solid through a composition variable. In the following, the switch of the variables can also be inspected in a more formal way: In the unary system, the molar volume of a certain phase $V_{m, u}^{\alpha} \equiv V^{\alpha} / n_{1}^{\alpha}$ reports how much volume $V^{\alpha}$ is occupied by the number of a single species $n_{1}^{\alpha}$ in this phase. In the corresponding binary system, however, molar volumes have the definition $V_{m, 1}^{\alpha} \equiv V_{1}^{\alpha} / n_{1}^{\alpha}$ and $V_{m, 2}^{\alpha} \equiv V_{2}^{\alpha} / n_{2}^{\alpha}$, where $n_{2}^{\alpha}$ are the moles of the second component (here vacancies) and $V_{1 / 2}^{\alpha}$ the corresponding volumes occupied by the species in the phase, such that the volume of a phase writes as $V^{\alpha}=$ $V_{1}^{\alpha}+V_{2}^{\alpha}$. According to the incompressibility and the way in which vacancies are introduced here, we may now set the molar volume independent of the phase and composition, such that $V_{m}=V_{m, 1}^{\alpha}=V_{m, 2}^{\alpha}=V_{m, 1}^{\beta}=V_{m, 2}^{\beta}$. One obtains the relation

$$
V_{m, u}^{\alpha}=\frac{V_{m}}{c_{1}^{\alpha}}
$$

for each phase. Inserting this into Eq. (A1) yields Eq. (A7), which shows that the notions of an incompressible binary atom-vacancy system with a varying composition and a unary solid-vapor system with varying molar volumes are interchangeable. To perform the switch, note that the characteristic variables are simply connected by the relation above.

\section{APPENDIX B: BALANCE LAW FOR AN EVOLVING SURFACE}

The evolution law for a surface can be derived from the mass balance. For a two-phase binary system, a derivation of the balance law is presented by Cermelli, Fried, and Gurtin [104]. The authors additionally consider a velocity field, due to the movement of the (possibly fluid) phases, which is absent in our problem. Therefore, any term considering the fluid velocity can be neglected. The corresponding final equation [104, Eq. (7.5)] writes as

$$
v_{n}\left(c^{+}-c^{-}\right)=\left(\boldsymbol{j}^{+}-\boldsymbol{j}^{-}\right) \cdot \boldsymbol{n}+\nabla_{s} \cdot \boldsymbol{j}_{s}+\stackrel{\circ}{\varsigma}-\varsigma v_{n} \kappa,
$$

where $v_{n}$ denotes the scalar normal velocity of the surface, $\boldsymbol{j}_{s}$ is the surface excess flux, $\boldsymbol{n}$ is the unit normal vector, $\boldsymbol{s}$ is the surface excess concentration, $\stackrel{\varsigma}{\zeta}$ represents its normal time derivative, and $\kappa$ denotes the curvature. If $r$ is the signed normal distance from the surface, the bulk fluxes from either side are expressed as $\boldsymbol{j}^{ \pm} \equiv \lim _{r \rightarrow 0 \pm} \boldsymbol{j}$, where $\boldsymbol{j}$ denotes the bulk flux. Similarly, the concentrations from either side, $c^{ \pm} \equiv$ $\lim _{r \rightarrow 0 \pm} c$, can be expressed as limits from the bulk. Given that bulk diffusion is absent the above equation simplifies to

$$
v_{n}\left(c^{+}-c^{-}\right)=\nabla_{s} \cdot \boldsymbol{j}_{s}+\stackrel{\circ}{\varsigma}-\varsigma v_{n} \kappa .
$$

A relation to the phase field might be obtained by letting $c$ be the interpolated composition $c(\boldsymbol{\phi}, \boldsymbol{\mu})=h_{\alpha}\left(\phi_{\alpha}\right) c^{\alpha}(\mu)+[1-$ $\left.h_{\alpha}\left(\phi_{\alpha}\right)\right] c^{\beta}(\mu)$, which is indeed a conserved quantity in the model. If $\alpha$ and $\beta$ are the phases in contact from the negative and positive side, one respectively obtains $c^{-}=c^{\alpha}\left(\mu^{-}\right)$and $c^{+}=c^{\beta}\left(\mu^{+}\right)$. Here $\mu^{ \pm} \equiv \lim _{r \rightarrow 0 \pm} \mu$ represents the chemical potential in the bulk on either side. What is interesting is the notion of an excess concentration, which reflects the possibility of adsorption in the interface, due to its physically nonzero thickness. According to the definition of the surface excess properties in [100, p. $179 \mathrm{ff}$.], the surface excess concentration in our phase-field model might be computed as an integral across the interface (at least as a first approximation):

$$
\begin{aligned}
\varsigma(s)= & \int_{r_{-}}^{0} c(r, s)-c^{\alpha}\left[\mu^{-}(s)\right] d r \\
& +\int_{0}^{r_{+}} c(r, s)-c^{\beta}\left[\mu^{+}(s)\right] d r
\end{aligned}
$$

where $r_{-} \equiv \epsilon \eta_{-}$and $r_{+} \equiv \epsilon \eta_{+}$denote the interfacial endpoints. The conclusion is that the integral in the sharp interface limit vanishes, as long as the composition remains bounded in the interface, since $r_{ \pm}=O(\epsilon)$. Therefore, one obtains $\lim _{\epsilon \rightarrow 0} \varsigma=0$. This is consistent with the argument given in [100, p. $197 \mathrm{ff}$.$] . The balance law in the sharp interface limit$ now writes as

$$
v_{n}=\frac{\nabla_{s} \cdot \boldsymbol{j}_{s}}{c^{\beta}\left(\mu^{+}\right)-c^{\alpha}\left(\mu^{-}\right)}
$$

\section{APPENDIX C: EXPANSION OF THE BOUNDARY CONDITION EQUATIONS}

\section{Phase-field boundary conditions}

At the endpoints $\eta_{ \pm}$, we expand the boundary condition equations in powers of $\epsilon$. Inherently, it is considered that $\eta_{ \pm}$ itself are functions of $\epsilon$. We start with the boundary conditions for the phase field (40) and (41)

$$
\phi_{\alpha}\left(\eta_{ \pm}, s\right)=C_{ \pm}
$$

where $C_{ \pm}$refer to constants $\left(C_{-}=1, C_{+}=0\right)$. We denote the left-hand side as $P$ and expand it in terms of $\epsilon$ as

$$
P=P(\epsilon=0)+\left.\epsilon \frac{\partial P}{\partial \epsilon}\right|_{\epsilon=0}+\left.\frac{\epsilon^{2}}{2} \frac{\partial^{2} P}{\partial \epsilon^{2}}\right|_{\epsilon=0}+O\left(\epsilon^{3}\right)=C_{ \pm}
$$

In this expanded form, it is easy to see that the relations $P(\epsilon=$ $0)=C_{ \pm}$and $\partial^{n} P /\left.\partial \epsilon^{n}\right|_{\epsilon=0}=0, \forall n>0$ have to hold, so as to satisfy the boundary condition. To find the corresponding 
expressions for these terms, $P$ is written as follows, in terms of the expansions (55) and (57):

$$
\begin{gathered}
P=\phi_{\alpha}^{0}\left(\eta_{ \pm}\right)+\epsilon \phi_{\alpha}^{1}\left(\eta_{ \pm}\right)+\epsilon^{2} \phi_{\alpha}^{2}\left(\eta_{ \pm}\right)+O\left(\epsilon^{3}\right)=C_{ \pm}, \\
\eta_{ \pm}=\eta_{ \pm}^{0}+\epsilon \eta_{ \pm}^{1}+\epsilon^{2} \eta_{ \pm}^{2}+O\left(\epsilon^{3}\right) .
\end{gathered}
$$

First, the following expression is easily obtained:

$$
P(\epsilon=0)=\phi_{\alpha}^{0}\left(\eta_{ \pm}^{0}\right)=C_{ \pm} .
$$

The other terms are found by first differentiating them, using the chain rule:

$$
\begin{gathered}
\frac{\partial P}{\partial \epsilon}=\frac{\partial \phi_{\alpha}^{0}\left(\eta_{ \pm}\right)}{\partial \epsilon}+\phi_{\alpha}^{1}\left(\eta_{ \pm}\right) \\
+\epsilon \frac{\partial \phi_{\alpha}^{1}\left(\eta_{ \pm}\right)}{\partial \epsilon}+2 \epsilon \phi_{\alpha}^{2}\left(\eta_{ \pm}\right)+O\left(\epsilon^{2}\right) \\
\frac{\partial^{2} P}{\partial \epsilon^{2}}=\frac{\partial^{2} \phi_{\alpha}^{0}\left(\eta_{ \pm}\right)}{\partial \epsilon^{2}}+2 \frac{\partial \phi_{\alpha}^{1}\left(\eta_{ \pm}\right)}{\partial \epsilon}+2 \phi_{\alpha}^{2}\left(\eta_{ \pm}\right)+O(\epsilon),
\end{gathered}
$$

which results in

$$
\begin{gathered}
\left.\frac{\partial P}{\partial \epsilon}\right|_{\epsilon=0}=\left.\frac{\partial \phi_{\alpha}^{0}\left(\eta_{ \pm}\right)}{\partial \epsilon}\right|_{\epsilon=0}+\phi_{\alpha}^{1}\left(\eta_{ \pm}^{0}\right), \\
\left.\frac{\partial^{2} P}{\partial \epsilon^{2}}\right|_{\epsilon=0}=\left.\frac{\partial^{2} \phi_{\alpha}^{0}\left(\eta_{ \pm}\right)}{\partial \epsilon^{2}}\right|_{\epsilon=0}+\left.2 \frac{\partial \phi_{\alpha}^{1}\left(\eta_{ \pm}\right)}{\partial \epsilon}\right|_{\epsilon=0}+2 \phi_{\alpha}^{2}\left(\eta_{ \pm}^{0}\right) .
\end{gathered}
$$

Additionally, the dependence on $\epsilon$, for any function $f\left(\eta_{ \pm}\right)$, may be rewritten as

$$
\begin{gathered}
\frac{\partial f\left(\eta_{ \pm}\right)}{\partial \epsilon}=\left.\frac{\partial f(\eta)}{\partial \eta}\right|_{\eta_{ \pm}} \frac{\partial \eta_{ \pm}}{\partial \epsilon} \\
\frac{\partial^{2} f\left(\eta_{ \pm}\right)}{\partial \epsilon^{2}}=\left.\frac{\partial^{2} f(\eta)}{\partial \eta^{2}}\right|_{\eta_{ \pm}}\left(\frac{\partial \eta_{ \pm}}{\partial \epsilon}\right)^{2}+\left.\frac{\partial f(\eta)}{\partial \eta}\right|_{\eta_{ \pm}} \frac{\partial^{2} \eta_{ \pm}}{\partial \epsilon^{2}}
\end{gathered}
$$

In the limit $\epsilon \rightarrow 0$, one accordingly obtains

$$
\begin{gathered}
\left.\frac{\partial f\left(\eta_{ \pm}\right)}{\partial \epsilon}\right|_{\epsilon=0}=\left.\frac{\partial f(\eta)}{\partial \eta}\right|_{\eta_{ \pm}^{0}} \eta_{ \pm}^{1}, \\
\left.\frac{\partial^{2} f\left(\eta_{ \pm}\right)}{\partial \epsilon^{2}}\right|_{\epsilon=0}=\left.\frac{\partial^{2} f(\eta)}{\partial \eta^{2}}\right|_{\eta_{ \pm}^{0}}\left(\eta_{ \pm}^{1}\right)^{2}+\left.2 \frac{\partial f(\eta)}{\partial \eta}\right|_{\eta_{ \pm}^{0}} \eta_{ \pm}^{2} .
\end{gathered}
$$

Inserting the result into the boundary conditions finally yields the sought terms

$$
\begin{aligned}
\left.\frac{\partial P}{\partial \epsilon}\right|_{\epsilon=0} & =\left.\frac{\partial \phi_{\alpha}^{0}(\eta)}{\partial \eta}\right|_{\eta_{ \pm}^{0}} \eta_{ \pm}^{1}+\phi_{\alpha}^{1}\left(\eta_{ \pm}^{0}\right)=0 \\
\left.\frac{\partial^{2} P}{\partial \epsilon^{2}}\right|_{\epsilon=0}= & \left.\frac{\partial^{2} \phi_{\alpha}^{0}(\eta)}{\partial \eta^{2}}\right|_{\eta_{ \pm}^{0}}\left(\eta_{ \pm}^{1}\right)^{2}+\left.2 \frac{\partial \phi_{\alpha}^{0}(\eta)}{\partial \eta}\right|_{\eta_{ \pm}^{0}} \eta_{ \pm}^{2} \\
& +\left.2 \frac{\partial \phi_{\alpha}^{1}(\eta)}{\partial \eta}\right|_{\eta_{ \pm}^{0}} \eta_{ \pm}^{1}+2 \phi_{\alpha}^{2}\left(\eta_{ \pm}^{0}\right)=0 .
\end{aligned}
$$

Expansions for the derivative boundary conditions, (42), can also be found when proceeding in the same manner.

\section{Flux boundary condition}

The balance law presented in Appendix A is utilized to derive flux boundary conditions at the interfacial endpoints $r_{ \pm}$. Accordingly, one starts with the more general Eq. (B1). Note that now the interfacial region is not treated as a whole, by incorporating excess properties, but the moving surface now corresponds solely to the coordinate $r_{ \pm}$. Moreover, since the isolines corresponding to $r_{ \pm}$have an exactly zero thickness mathematically, which makes them independent from $\epsilon$, one can safely neglect any surface excess properties. Thus, one obtains two equations for either end of the interface, which read as

$$
\begin{aligned}
& v_{n}\left[c^{+}-c\left(\eta_{+}\right)\right]=\left[\boldsymbol{j}_{b}^{+}-\boldsymbol{j}_{i}\left(\eta_{+}\right)\right] \cdot \boldsymbol{n}, \\
& v_{n}\left[c\left(\eta_{-}\right)-c^{-}\right]=\left[\boldsymbol{j}_{i}\left(\eta_{-}\right)-\boldsymbol{j}_{b}^{-}\right] \cdot \boldsymbol{n} .
\end{aligned}
$$

It is important to be careful with the limits, where $c\left(\eta_{ \pm}\right)$, for example, denotes the composition at the interfacial endpoints, approached from the inner region, in contrast to the outer limits $c^{ \pm}$, which must not necessarily equal each other. Similarly, $\boldsymbol{j}_{b}^{ \pm}$are the bulk fluxes at either side and $\boldsymbol{j}_{i}^{ \pm}$are the interfacial ones. In the absence of bulk diffusion, one obtains

$$
\begin{aligned}
& j_{i, n}\left(\eta_{+}\right)=v_{n}\left[c\left(\eta_{+}\right)-c^{+}\right], \\
& j_{i, n}\left(\eta_{-}\right)=v_{n}\left[c\left(\eta_{-}\right)-c^{-}\right],
\end{aligned}
$$

where $j_{i, n}\left(\eta_{ \pm}\right) \equiv \boldsymbol{j}_{i}\left(\eta_{ \pm}\right) \cdot \boldsymbol{n}$ are the scalar normal fluxes in the interface at either side. In the sharp-interface limit $\epsilon \rightarrow 0$, the above equations can be written as

$$
\begin{aligned}
& \left.j_{i, n}\left(\eta_{+}^{0}\right)\right|_{\epsilon=0}=v_{n}^{0}\left[c\left(\eta_{+}^{0}, \epsilon=0\right)-c^{+}\right], \\
& \left.j_{i, n}\left(\eta_{-}^{0}\right)\right|_{\epsilon=0}=v_{n}^{0}\left[c\left(\eta_{-}^{0}, \epsilon=0\right)-c^{-}\right] .
\end{aligned}
$$

In the absence of bulk diffusion, we first note that the scalar normal flux in the current model writes as

$$
j_{i, n}=-\frac{M}{\epsilon} \frac{\partial \mu}{\partial r}=-\frac{M}{\epsilon^{2}} \frac{\partial \mu}{\partial \eta},
$$

according to Eq. (25) and since $r=\epsilon \eta$.

With $J \equiv M \partial \mu / \partial \eta$, one obtains

$$
J\left(\eta_{ \pm}\right)=-\epsilon^{2} j_{i, n}\left(\eta_{ \pm}\right)
$$

Since $j_{i, n}\left(\eta_{ \pm}\right)=O(1)$, this results in $J\left(\eta_{ \pm}, \epsilon=0\right)=0$ and $\partial J\left(\eta_{ \pm}\right) /\left.\partial \epsilon\right|_{\epsilon=0}=0$, as well as $\partial^{n} J\left(\eta_{ \pm}\right) /\left.\partial \epsilon^{n}\right|_{\epsilon=0}=$ $-\partial^{n} \epsilon^{2} j_{i, n}\left(\eta_{ \pm}\right) /\left.\partial \epsilon^{n}\right|_{\epsilon=0}, \forall n \geqslant 2$. It is recalled that one can write

$$
J=J^{0}+\epsilon J^{1}+\epsilon^{2} J^{2}+O\left(\epsilon^{3}\right),
$$

where $J^{0} \equiv M^{0} \frac{\partial \mu^{0}}{\partial \eta}, J^{1} \equiv M^{0} \frac{\partial \mu^{1}}{\partial \eta}+M^{1} \frac{\partial \mu^{0}}{\partial \eta}, J^{2} \equiv M^{0} \frac{\partial \mu^{2}}{\partial \eta}+$ $M^{1} \frac{\partial \mu^{1}}{\partial \eta}+M^{2} \frac{\partial \mu^{0}}{\partial \eta}$. To resolve the conditions stated above, the derivatives with respect to $\epsilon$ are now written as follows in a straightforward manner:

$$
\begin{aligned}
\frac{\partial J}{\partial \epsilon} & =\frac{\partial J^{0}}{\partial \epsilon}+J^{1}+\epsilon \frac{\partial J^{1}}{\partial \epsilon}+2 \epsilon J^{2}+O\left(\epsilon^{2}\right), \\
\frac{\partial^{2} J}{\partial \epsilon^{2}} & =\frac{\partial^{2} J^{0}}{\partial \epsilon^{2}}+2 \frac{\partial J^{1}}{\partial \epsilon}+2 J^{2}+O(\epsilon) .
\end{aligned}
$$

The expressions obtained for the first three orders, by the use of Eqs. (C12) and (C13) as well as Eqs. (C20) and (C21), 
are

$$
\begin{gathered}
0=J^{0}\left(\eta_{ \pm}^{0}\right), \\
0=\left.\frac{\partial J^{0}}{\partial \eta}\right|_{\eta_{ \pm}^{0}} \eta_{ \pm}^{1}+J^{1}\left(\eta_{ \pm}^{0}\right), \\
0=\left.\frac{\partial^{2} J^{0}}{\partial \eta^{2}}\right|_{\eta_{ \pm}^{0}}\left(\eta_{ \pm}^{1}\right)^{2}+\left.2 \frac{\partial J^{0}}{\partial \eta}\right|_{\eta_{ \pm}^{0}} \eta_{ \pm}^{2}+\left.2 \frac{\partial J^{1}}{\partial \eta}\right|_{\eta_{ \pm}^{0}} \eta_{ \pm}^{1} \\
+2 J^{2}\left(\eta_{ \pm}^{0}\right)+2 v_{n}^{0}\left[c\left(\eta_{ \pm}^{0}, \epsilon=0\right)-c^{ \pm}\right] .
\end{gathered}
$$

\section{APPENDIX D: BOUNDEDNESS OF THE FIRST-ORDER PHASE-FIELD CORRECTION}

The generic solution of Eq. (70), which satisfies $\phi_{\alpha}^{1}(\eta=$ $0)=0$, is

$$
\begin{aligned}
\phi_{\alpha}^{1}(\eta)= & \frac{1}{k} \sin (k \eta) \int_{0}^{\eta} q(\eta) \cos (k \eta) d \eta \\
& -\frac{1}{k} \cos (k \eta) \int_{0}^{\eta} q(\eta) \sin (k \eta) d \eta+T \sin (k \eta),
\end{aligned}
$$

with $k$ and $q(\eta)$ standing for $4 / \pi$ and the right-hand side of Eq. (70), respectively, where $T$ is a real constant. The derivative turns out to be

$$
\begin{aligned}
\frac{\partial \phi_{\alpha}^{1}(\eta)}{\partial \eta}= & \cos (k \eta) \int_{0}^{\eta} q(\eta) \cos (k \eta) d \eta \\
& +\sin (k \eta) \int_{0}^{\eta} q(\eta) \sin (k \eta) d \eta+T k \cos (k \eta) .
\end{aligned}
$$

Since $q(\eta)$ is bounded, the above derivative is bounded at $\eta_{-}^{0}$ and $\eta_{+}^{0}$, which is necessary to infer Eq. (74) from Eq. (73).

\section{APPENDIX E: BOUNDEDNESS OF THE SECOND-ORDER CHEMICAL POTENTIAL}

We will solve the second-order correction of the chemical potential for a particular example, $h_{\alpha}=\phi_{\alpha}$ and $M\left(\phi_{\alpha}\right)=$ $\bar{M} \sqrt{\phi_{\alpha}\left(1-\phi_{\alpha}\right)}$. The starting point is Eq. (89), which is integrated twice. Taking the boundary conditions into account, one obtains

$$
\begin{aligned}
\mu^{2}= & \mu_{A}^{2} \ln \left[\cos \left(\frac{4}{\pi} \eta\right)\right] \\
& +\mu_{B}^{2} \ln \left[\tan \left(\frac{4}{\pi} \eta\right)+\sec \left(\frac{4}{\pi} \eta\right)\right]+\mu_{C}^{2},
\end{aligned}
$$

where the coefficients read as $\mu_{A}^{2} \equiv v_{n}^{0} \pi\left(\Delta c_{b i}^{\alpha}-\right.$ $\left.\Delta c_{b i}^{\beta}\right) /\left(4 \bar{M}^{\alpha \beta}\right)$ and $\mu_{B}^{2} \equiv v_{n}^{0} \pi\left(\Delta c_{b i}^{\alpha}+\Delta c_{b i}^{\beta}\right) /\left(4 \bar{M}^{\alpha \beta}\right)$, and
$\mu_{C}^{2}$ is an integration constant. $\Delta c_{b i}^{\alpha} \equiv c^{\alpha}\left(\mu^{-}\right)-c^{\alpha}\left(\mu^{0}\right)$ and $\Delta c_{b i}^{\beta} \equiv c^{\beta}\left(\mu^{+}\right)-c^{\beta}\left(\mu^{0}\right)$ are the differences in the composition between the bulk and the interface at either side of the interface. Interestingly, $\mu^{2}$ shows a nontrivial variation across the interface. Moreover, it is especially not bounded at the interfacial endpoints $\eta_{ \pm}^{0}$. One may now ask whether the chemical potential is bounded at all. However, this question cannot be answered only by dealing with the second-order Taylor coefficient of the chemical potential, in terms of $\epsilon$. A similar problem arose in a recent derivation of the Cahn-Hilliard equation with a quadratic mobility [45]. Please note the similarity between Eq. (92) and [45, cf. Eq. (4.30)]. In their set of equations, based on a well potential, an exponentially growing third-order chemical potential correction was observed. Since we are dealing with a finite domain, instead of a function, which is going to $\infty$ in the limit $\eta \rightarrow \infty$ (where $c$ tends toward the bulk value), it is no surprise that we already observe the unbounded nature after a finite length, where the bulk is approached. In the above-mentioned work, the authors did resolve the problem by introducing another boundary layer and showed that the unboundedness went away after carefully matching with the inner region. Please note that for the derivation of the governing equations, it was not necessary to perform such an extra step. However, it helped to understand the exponential term and allowed for a proper matching with the outer region. Regarding our problem, we will therefore only briefly review the properties of a possible additional layer and will reserve the (possibly nontrivial) extra matching step for a future work. Thus, we perform expansions of $\mu^{2}$, at the interfacial endpoints, and obtain the relations $\lim _{\eta \rightarrow \eta_{+}^{0}} \mu^{2}=\left(\mu_{A}^{2}-\mu_{B}^{2}\right) \ln \left(\eta_{+}^{0}-\eta\right)$ and $\lim _{\eta \rightarrow \eta_{-}^{0}} \mu^{2}=\left(\mu_{A}^{2}+\mu_{B}^{2}\right) \ln \left(\eta-\eta_{-}^{0}\right)$. The additional layer can be regarded as the region where the correction $\epsilon^{2} \mu^{2}$ contributes an amount in the same order of magnitude as the correction with one order below, $O(\epsilon)$. Therefore, we search for the position $\eta$, at a fixed $\epsilon$, for which $\left|\epsilon \mu^{2}\right|>\chi$, where $\chi$ is a small positive constant. The resultant relations are $\Delta \eta_{ \pm}<\exp \left[-\chi /\left(\epsilon\left|\mu_{A}^{2} \mp \mu_{B}^{2}\right|\right)\right]$, where $\Delta \eta_{ \pm}=\left|\eta-\eta_{ \pm}^{0}\right|$ denotes the width of the additional layer. Therefore, the width of the layer decays exponentially with $\epsilon$, such that it will become negligibly small relatively quickly, which might be the reason why the chemical potential appears to be discontinuous in the presented results. Preliminary numerical tests for high composition differences and high interface velocities at a large $\epsilon$ reveal that the chemical potential remains bounded very well and qualitatively supports the existence of a boundary layer-like behavior, close to the interfacial endpoints, where the chemical potential changed rapidly, but continuously from the bulk to the interfacial value at the front.
[1] L. Q. Chen, Annu. Rev. Mater. Sci. 32, 113 (2002).

[2] W. J. Boettinger, J. A. Warren, C. Beckermann, and A. Karma, Annu. Rev. Mater. Sci. 32, 163 (2002).

[3] I. Steinbach, Modell. Simul. Mater. Sci. Eng. 17, 073001 (2009).
[4] M. Plapp, Philos. Mag. 91, 25 (2011).

[5] S. M. Allen and J. W. Cahn, Acta Metall. 27, 1085 (1979).

[6] J. W. Cahn and J. E. Hilliard, J. Chem. Phys. 28, 258 (1958).

[7] G. Caginalp, Arch. Ration. Mech. Anal. 92, 205 (1986).

[8] R. Kobayashi, Physica D 63, 410 (1993). 
[9] A. Karma and W.-J. Rappel, Phys. Rev. E 57, 4323 (1998).

[10] S. G. Kim, W. T. Kim, and T. Suzuki, Phys. Rev. E 60, 7186 (1999).

[11] B. Echebarria, R. Folch, A. Karma, and M. Plapp, Phys. Rev. E 70, 061604 (2004).

[12] J. C. Ramirez, C. Beckermann, A. Karma, and H.-J. Diepers, Phys. Rev. E 69, 051607 (2004).

[13] J. Eiken, B. Böttger, and I. Steinbach, Phys. Rev. E 73, 066122 (2006).

[14] M. Plapp, Phys. Rev. E 84, 031601 (2011).

[15] A. Choudhury and B. Nestler, Phys. Rev. E 85, 021602 (2012).

[16] I. Steinbach, F. Pezzolla, B. Nestler, M. Seeßelberg, R. Prieler, G. J. Schmitz, and J. L. Rezende, Physica D 94, 135 (1996).

[17] I. Steinbach and F. Pezzolla, Physica D 134, 385 (1999).

[18] B. Nestler, H. Garcke, and B. Stinner, Phys. Rev. E 71, 041609 (2005).

[19] W. W. Mullins, J. Appl. Phys. 28, 333 (1957).

[20] W. M. Robertson, J. Appl. Phys. 42, 463 (1971).

[21] J. Cahn and J. Taylor, Acta Metall. Mater. 42, 1045 (1994).

[22] J. W. Cahn, C. M. Elliott, and A. Novick-Cohen, Eur. J. Appl. Math. 7, 287 (1996).

[23] S. Puri, A. J. Bray, and J. L. Lebowitz, Phys. Rev. E 56, 758 (1997).

[24] J. Zhu, L.-Q. Chen, J. Shen, and V. Tikare, Phys. Rev. E 60, 3564 (1999).

[25] D. Seol, S. Hu, Z. Liu, L. Chen, S. Kim, and K. Oh, J. Appl. Phys. 98, 044910 (2005).

[26] J. Kim, Commun. Nonlinear Sci. Numer. Simul. 12, 1560 (2007).

[27] S. Torabi, J. Lowengrub, A. Voigt, and S. Wise, Proc. R. Soc. A 465, 1337 (2009).

[28] W. Jiang, W. Bao, C. V. Thompson, and D. J. Srolovitz, Acta Mater. 60, 5578 (2012).

[29] S. Dai and Q. Du, J. Comput. Phys. 310, 85 (2016).

[30] J. Eggleston and P. W. Voorhees, Appl. Phys. Lett. 80, 306 (2002).

[31] D. Yeon, P. Cha, and M. Grant, Acta Mater. 54, 1623 (2006).

[32] A. Rätz, A. Ribalta, and A. Voigt, J. Comput. Phys. 214, 187 (2006).

[33] A. M. Lacasta, A. Hernández-Machado, J. M. Sancho, and R. Toral, Phys. Rev. B 45, 5276 (1992).

[34] A. M. Lacasta, J. M. Sancho, A. Hernández-Machado, and R. Toral, Phys. Rev. B 48, 6854 (1993).

[35] A. J. Bray and C. L. Emmott, Phys. Rev. B 52, R685(R) (1995).

[36] M. Naffouti, R. Backofen, M. Salvalaglio, T. Bottein, M. Lodari, A. Voigt, T. David, A. Benkouider, I. Fraj, L. Favre et al., Sci. Adv. 3, eaao1472 (2017).

[37] M. Salvalaglio, R. Backofen, and A. Voigt, Phys. Rev. B 94, 235432 (2016).

[38] M. Salvalaglio, R. Backofen, A. Voigt, and F. Montalenti, Nanoscale Res. Lett. 12, 554 (2017).

[39] M. Albani, L. Ghisalberti, R. Bergamaschini, M. Friedl, M. Salvalaglio, A. Voigt, F. Montalenti, G. Tütüncüoglu, A. Fontcubertai Morral, and L. Miglio, Phys. Rev. Mater. 2, 093404 (2018).

[40] M. Mahadevan and R. M. Bradley, Physica D 126, 201 (1999).

[41] D. N. Bhate, A. Kumar, and A. F. Bower, J. Appl. Phys. 87, 1712 (2000).
[42] L. Baňas and R. Nürnberg, Comput. Visualization Sci. 12, 319 (2009).

[43] S. Dai and Q. Du, Multiscale Model. Simul. 12, 1870 (2014).

[44] A. A. Lee, A. Münch, and E. Süli, Appl. Phys. Lett. 107, 081603 (2015).

[45] A. Lee, A. Münch, and E. Süli, SIAM J. Appl. Math. 76, 433 (2016).

[46] M. Dziwnik, A. Münch, and B. Wagner, Nonlinearity 30, 1465 (2017).

[47] C. Gugenberger, R. Spatschek, and K. Kassner, Phys. Rev. E 78, 016703 (2008).

[48] W. B. Andrews, K. L. Elder, P. W. Voorhees, and K. Thornton, Phys. Rev. Mater. 4, 103401 (2020).

[49] A. Voigt, Appl. Phys. Lett. 108, 036101 (2016).

[50] A. A. Lee, A. Münch, and E. Süli, Appl. Phys. Lett. 108, 036102 (2016).

[51] B. Li, J. Lowengrub, A. Ratz, and A. Voigt, Commun. Comput. Phys. 6, 433 (2009).

[52] Y. U. Wang, Acta Mater. 54, 953 (2006).

[53] F. Abdeljawad, D. S. Bolintineanu, A. Cook, H. BrownShaklee, C. DiAntonio, D. Kammler, and A. Roach, Acta Mater. 169, 60 (2019).

[54] N. Moelans, B. Blanpain, and P. Wollants, in Solid State Phenomena, edited by R. Abdank-Kozubski, G. E. Murch, and P. Zięba (Trans Tech Publications Ltd., Switzerland, 2007), Vol. 129, pp. 89-94.

[55] A. Mukherjee, Electric field-induced directed assembly of diblock copolymers and grain boundary grooving in metal interconnects, Ph.D. thesis, Karlsruher Institut für Technologie (KIT), 2019.

[56] P. Jacquet, R. Podor, J. Ravaux, J. Lautru, J. Teisseire, I. Gozhyk, J. Jupille, and R. Lazzari, Acta Mater. 143, 281 (2018).

[57] K. Ahmed, C. A. Yablinsky, A. Schulte, T. Allen, and A. El-Azab, Modell. Simul. Mater. Sci. Eng. 21, 065005 (2013).

[58] K. Ahmed, T. Allen, and A. El-Azab, J. Mater. Sci. 51, 1261 (2016).

[59] J. Barrett, H. Garcke, and R. Nürnberg, Interfaces Free Bound. 9, 171 (2007).

[60] A. Novick-Cohen, Physica D 137, 1 (2000).

[61] A. Novick-Cohen and L. P. Hari, Physica D 209, 205 (2005).

[62] A. Kazaryan, Y. Wang, and B. R. Patton, Scr. Mater. 41, 487 (1999).

[63] K. Asp and J. Ågren, Acta Mater. 54, 1241 (2006).

[64] R. Mukherjee, T. Chakrabarti, E. A. Anumol, T. A. Abinandanan, and N. Ravishankar, ACS Nano 5, 2700 (2011).

[65] T. Chakrabarti and R. Mukherjee, Comput. Mater. Sci. 169, 109115 (2019).

[66] M. R. Tonks, Y. Zhang, A. Butterfield, and X.-M. Bai, Modell. Simul. Mater. Sci. Eng. 23, 045009 (2015).

[67] S. Biner, Modell. Simul. Mater. Sci. Eng. 24, 035019 (2016).

[68] J. Kundin, H. Sohaib, R. Schiedung, and I. Steinbach, Modell. Simul. Mater. Sci. Eng. 26, 065003 (2018).

[69] A. Mukherjee, K. Ankit, R. Mukherjee, and B. Nestler, J. Electron. Mater. 45, 6233 (2016).

[70] A. Mukherjee, K. Ankit, M. Selzer, and B. Nestler, Phys. Rev. Appl. 9, 044004 (2018).

[71] R. Davis, F. Abdeljawad, J. Lillibridge, and M. Haataja, Acta Mater. 78, 271 (2014). 
[72] Y. Yang, O. Ragnvaldsen, Y. Bai, M. Yi, and B.-X. Xu, npj Comput. Mater. 5, 1 (2019).

[73] L. Amirouche and M. Plapp, Acta Mater. 57, 237 (2009).

[74] S. Chakraborty, P. Kumar, and A. Choudhury, Acta Mater. 153, 377 (2018).

[75] J. Hötzer, M. Seiz, M. Kellner, W. Rheinheimer, and B. Nestler, Acta Mater. 164, 184 (2019).

[76] I. Greenquist, M. R. Tonks, L. K. Aagesen, and Y. Zhang, Comput. Mater. Sci. 172, 109288 (2020).

[77] I. Greenquist, M. Tonks, M. Cooper, D. Andersson, and Y. Zhang, J. Nucl. Mater. 532, 152052 (2020).

[78] A. Choudhury, M. Kellner, and B. Nestler, Curr. Opin. Solid State Mater. Sci. 19, 287 (2015).

[79] F. J. Humphreys and M. Hatherly, Recrystallization and Related Annealing Phenomena, 2nd ed. (Elsevier, 2004).

[80] A. A. Wheeler, W. J. Boettinger, and G. B. McFadden, Phys. Rev. A 45, 7424 (1992).

[81] J. Hötzer, P. Steinmetz, M. Jainta, S. Schulz, M. Kellner, B. Nestler, A. Genau, A. Dennstedt, M. Bauer, H. Köstler, and U. Rüde, Acta Mater. 106, 249 (2016).

[82] M. Kellner, I. Sprenger, P. Steinmetz, J. Hötzer, B. Nestler, and M. Heilmaier, Comput. Mater. Sci. 128, 379 (2017).

[83] P. Steinmetz, M. Kellner, J. Hötzer, and B. Nestler, Metall. Mater. Trans. B 49, 213 (2018).

[84] S. Ghosh, A. Karma, M. Plapp, S. Akamatsu, S. BottinRousseau, and G. Faivre, Acta Mater. 175, 214 (2019).

[85] T. Mittnacht, P. G. Kubendran Amos, D. Schneider, and B. Nestler, in Proceedings of the 1st International Conference on Numerical Modelling in Engineering, edited by M. A. Wahab (Springer, Singapore, 2019), pp. 290-299.

[86] P. G. Kubendran Amos, E. Schoof, D. Schneider, and B. Nestler, in Proceedings of the 1st International Conference on Numerical Modelling in Engineering, edited by M. A. Wahab (Springer, Singapore, 2019), pp. 47-63.

[87] L. Mushongera, P. G. Kubendran Amos, B. Nestler, and K. Ankit, Acta Mater. 150, 78 (2018).

[88] L. Mushongera, P. G. Kubendran Amos, E. Schoof, P. Kumar, and B. Nestler, J. Mater. Sci. 55, 5280 (2020).

[89] P. G. Kubendran Amos, E. Schoof, D. Schneider, and B. Nestler, J. Alloys Compd. 767, 1141 (2018).

[90] P. G. Kubendran Amos, E. Schoof, N. Streichan, D. Schneider, and B. Nestler, Comput. Mater. Sci. 159, 281 (2019).

[91] P. G. Kubendran Amos, E. Schoof, D. Schneider, and B. Nestler, Acta Mater. 159, 51 (2018).

[92] P. G. Kubendran Amos, A. Bhattacharya, B. Nestler, and K. Ankit, Acta Mater. 161, 400 (2018).

[93] P. G. Kubendran Amos, L. Mushongera, and B. Nestler, Comput. Mater. Sci. 144, 363 (2018).

[94] P. G. Kubendran Amos, E. Schoof, J. Santoki, D. Schneider, and B. Nestler, Comput. Mater. Sci. 173, 109388 (2020).

[95] P. G. Kubendran Amos, R. Perumal, M. Selzer, and B. Nestler, J. Mater. Sci. Technol. 45, 215 (2020).

[96] T. Mittnacht, P. K. Amos, D. Schneider, and B. Nestler, J. Mater. Sci. Technol. 77, 252 (2021).

[97] C. Herring, Phys. Powder Metall. 1, 143 (1951).
[98] R. T. DeHoff, Thermodynamics in Materials Science, 2nd ed. (Taylor \& Francis, Boca Raton, FL, 2003).

[99] J. C. Slattery, L. Sagis, and E.-S. Oh, Interfacial Transport Phenomena (Springer US, Boston, 2007).

[100] G. M. Mavrovouniotis and H. Brenner, Philos. Trans. R. Soc. London, Ser. A 345, 165 (1993).

[101] G. B. McFadden, A. A. Wheeler, and D. M. Anderson, Physica D 144, 154 (2000).

[102] P. G. Kubendran Amos, Understanding the volume-diffusion governed shape-instabilities in metallic systems, Ph.D. thesis, Karlsruher Institut für Technologie (KIT), 2019.

[103] R. Folch, J. Casademunt, A. Hernández-Machado, and L. Ramírez-Piscina, Phys. Rev. E 60, 1724 (1999).

[104] P. Cermelli, E. Fried, and M. E. Gurtin, J. Fluid Mech. 544, 339 (2005).

[105] W. Mullins, Trans. AIME 218, 354 (1960).

[106] W. Mullins and P. Shewmon, Acta Metall. 7, 163 (1959).

[107] B. Zhao and L. Shvindlerman, Int. Polym. Process. 19, 424 (2004).

[108] E. Rabkin and L. Klinger, Mater. Sci. Technol. 17, 772 (2001).

[109] P. Sachenko, J. Schneibel, J. Swadener, and W. Zhang, Philos. Mag. Lett. 80, 627 (2000).

[110] P. Sachenko, J. H. Schneibel, and W. Zhang, Philos. Mag. A 82, 815 (2002).

[111] W. Zhang, P. Sachenko, and J. H. Schneibel, J. Mater. Res. 17, 1495 (2002).

[112] P. Sachenko, J. Schneibel, and W. Zhang, Scr. Mater. 50, 1253 (2004).

[113] W. Zhang, P. Sachenko, and J. Schneibel, Defect Diffus. Forum 233-234, 149 (2004).

[114] W. Zhang and I. Gladwell, J. Cryst. Growth 277, 608 (2005).

[115] D. M. Saylor and G. S. Rohrer, J. Am. Ceram. Soc. 82, 1529 (1999).

[116] W. Mullins, Acta Metall. 6, 414 (1958).

[117] V. Aristov, V. Fradkov, and L. Shvindlerman, Phys. Met. Metallogr. 45, 83 (1978).

[118] S. Hackney, Scr. Mater. 35, 29 (1996).

[119] F. Yang, Comput. Mater. Sci. 7, 279 (1997).

[120] J. Kanel, A. Novick-Cohen, and A. Vilenkin, Acta Mater. 51, 1981 (2003).

[121] V. A. Ivanov, D. A. Molodov, L. S. Shvindlerman, and G. Gottstein, Acta Mater. 52, 969 (2004).

[122] T. Wang, G. Sheng, Z.-K. Liu, and L.-Q. Chen, Acta Mater. 56, 5544 (2008).

[123] U. Grafe, B. Böttger, J. Tiaden, and S. Fries, Scr. Mater. 42, 1179 (2000).

[124] Q. Chen, N. Ma, K. Wu, and Y. Wang, Scr. Mater. 50, 471 (2004).

[125] H. Gleiter, Acta Metall. 27, 187 (1979).

[126] Y. Estrin and K. Lücke, Acta Metall. 29, 791 (1981).

[127] K. Lücke and G. Gottstein, Acta Metall. 29, 779 (1981).

[128] M. Upmanyu, D. J. Srolovitz, L. S. Shvindlerman, and G. Gottstein, Interface Sci. 6, 289 (1998). 